\title{
BERNSTEIN'S INEQUALITY \\ FOR \\ LOCALLY COMPACT GROUPS
}

by

Walter R. Bloom

A thesis submitted for the degree of Doctor of Philosophy at the

Australian National University Canberra

February, 1974 


\section{STATEMENT}

Except where specifically stated otherwise the results in this thesis are the product of my own research.

Walter R. Bloom 


\section{ACKNOWLEDGEMENTS}

During the work for this thesis I held a Commonwealth Postgraduate Research Award. I would like to thank the Department of Education, Canberra and the Australian National University for their generous financial assistance.

I would also like to thank my wife, Lyn, for her constant encouragement in the face of seemingly insuperable problems.

My greatest thanks are due to my supervisor, Professor Robert E. Edwards, for his patient and expert guidance during the research for this thesis.

Finally I thank Mrs Barbara Geary for her excellent typing. 


\section{ERRATA AND COMMENTS}

p. 10 line 12

denotes the empty set.

p. 14 lines $7^{-}-1^{-}$

$g w^{2} \in L^{1}(G)$.

If $g \in L_{w^{2}}^{1} \cap C_{0}(G)$ then $g^{2} w^{2}=g \cdot g w^{2} \in L^{1}(G)$ since $g \in C_{0}(G)$,

\section{p. 27 Section 1.5}

$\Gamma$ always contains compactly generated open subgroups. Hence Corollary 1.5 .2 holds for a $11 \Gamma$ since, by Hewitt and Ross [1], (5.14), there is a compactly generated open subgroup $\Omega$ of $\Gamma$ containing $\Upsilon$ (in which case the $\Delta$ in Theorem 1.5.1 is void).

p. 29 Tines 15,20

p. 30 lines $7,8,11,12$

Wherever $\operatorname{Tr}[\cdot]$ occurs, it should be replaced by $d\left(U_{s}\right) \operatorname{Tr}[\cdot]$.

p. 35 lines 7,8

Kronecker's theorem is not needed since

$$
\begin{aligned}
\left\|_{a} f-f\right\|_{\infty} & =\left\|\alpha\left(\exp \left(-i n_{s} a\right)-1\right) e_{n_{s}}+\beta(\exp (-i m a)-1) e_{m}\right\|_{\infty} \\
& =\| \alpha\left(\exp \left(-i n_{s} a\right)-1\right) e_{n_{s}-m}+\beta\left(\exp (-i m a)-1 \|_{\infty}\right.
\end{aligned}
$$

and, as $n_{s}-m \neq 0$, this last quantity is

$$
|\alpha|\left|\exp \left(-i n_{s} \alpha\right)-1\right|+|\beta||\exp (-i m \alpha)-1| \text {. }
$$

Similarly for p. 36 lines $9^{-}, 8^{-}$. 
p. 49

The proof of Lemma 3.1.1 follows immediately from Hewitt and Ross [1], (24.38) and the fact that $\Lambda$ is the adjoint of $\rho$.

p. 59 line $9^{-}$

Replace $(5.40)(d)$ by $(5.11)$.

p. 80 lines 7-15

Since the ball $\left\{g \in L^{p}(G):\|g\|_{p} \leq m\right\}$ is strongly closed and convex, it is weakly closed.

p. 81 line $8^{-}$

$f_{1}$ is not needed. Choose $\alpha \neq 0$ so that $\|\alpha k\|_{q}<\delta$. Then $f_{0}$, $f_{0}+\alpha k$ belong to $V$ and so there exist $g_{1}, g_{2} \in L^{p}(G)$ such that $\hat{f}_{0}-\hat{g}_{1}$ and $\left(f_{0}+\alpha k\right) \hat{-} \hat{g}_{2}$ vanish on $\Omega_{n_{0}}$. Now proceed as on $p .82$ 


\section{ABSTRACT}

An extended form of a famous inequality of $S . N$. Bernstein states that for a trigonometric polynomial $T_{n}$ of degree at most $n$

$$
\left\|T_{n}^{\prime}\right\|_{p} \leq n\left\|T_{n}\right\|_{p}
$$

where $T^{\prime}$ denotes the derivative of $T_{n}$ and $I \leq p \leq \infty$. There is also a corresponding statement for functions on the real line which are extendible into the complex plane to functions of exponential type.

This thesis is concerned with versions of Bernstein's inequality for pth-power integrable functions on locally compact Hausdorff Abelian and compact Hausdorff groups. The inequalities obtained are of the form

$$
\|f-f\|_{p} \leq \omega(\alpha)\|f\|_{p}
$$

where $f \in L^{P}(G)$ has spectrum contained in a given relatively compact set $T, a f$ denotes the left $a$-translate of $f$, and $\lim _{a \rightarrow 0} \omega(a)=0$. Chapter 1 deals with this problem for various choices of $G$ and $T$. In Chapter 2 it is shown that if $S$ is a translation-invariant subspace of $L^{p}(G)$ with every $f \in S$ satisfying (1), then $U\{\Sigma(f): f \in S\}$ is relatively compact. This result can be considered as a converse to that dealt with in the first chapter. A more classical version of the inequality is examined in Chapter 3, namely

$$
\left\|D_{\rho}^{p} f\right\|_{p} \leq k\|f\|_{p}
$$

here $\rho$ is a continuous homomorphism of $R$ into $G$ and

$$
D_{\rho}^{p} f=\lim _{r \rightarrow 0} r^{-1}(-\rho(r) f-f)
$$

(where the limit is taken in the $L^{p}$-sense) is the $L^{p}$-derivative of $f$ along $\rho$. Initially it is proved that if $f \in L^{p}(G)$ and $\Sigma(f) \subset T$ (where $\Upsilon$ is relatively compact) then $f$ is $L^{p}$-differentiable along $\rho$ for any $\rho$. Inequality (2) then follows readily with $k=\kappa(\rho, T)$. There is also a converse result. 


\section{CONTENTS}

STATEMENT

ACKNOWLEDGEMENTS

ABSTRACT

CONTENTS

CHAPTER 0 INTRODUCTION AND PRELIMINARIES

0.0 Introduction and background to Bernstein's inequality

0.1 General references

0.2 Definitions and notation

CHAPTER I BERNSTEIN'S INEQUALITY FOR LOCALLY COMPACT GROUPS

1.0 Introduction

1.1 Estimates for $\beta_{\Upsilon}^{L}(\alpha)$ on locally compact Abelian groups

1.2 Estimates for $\beta_{T}^{L}(\alpha)$ when $L$ is a *-module over a weighted $L^{1}$-space

1.3 Estimates for $\beta_{T}^{p}(\alpha)$ on locally compact Abelian groups

1.4 Further estimates for $\beta_{\Upsilon}^{\infty}(\alpha)$ on locally compact Abelian groups

1.5 The case where $\Gamma$ contains a compactly generated open subgroup

1.6 An estimate for $\beta_{T}^{p}(\alpha)$ on compact groups

1.7 An application

CHAPTER 2 A CONVERSE OF BERNSTEIN'S INEQUALITY FOR LOCALLY COMPACT GROUPS

2.0 Introduction

2.1 The case where $G$ is Abelian

2.2 A more refined result for $L^{I}(G)$ and $C_{u}(G)$ 
CHAPTER 3 A DERIVATIVE FORM OF BERNSTEIN'S INEQUALITY AND ITS

CONVERSE FOR LOCALLY COMPACT ABELIAN GROUPS

3.0 Introduction

3.I Bernstein's inequality for differentiation along a one-parameter subgroup

3.2 A converse result

3.3 A necessary and sufficient condition for certain $\left\{\rho_{c}\right\}_{\iota \in I}$ to be jointly-proper

APPENDIX A SOME RESULTS ON THE FOURIER TRANSFORM AND SPECTRUM OF

$$
\text { AN } \quad L^{p}-\text { FUNCTION }
$$

A.1 Some properties of the Fourier transform

A.2 The equivalence of two definitions of spectrum

A.3 Strict local inclusion results between spaces of Fourier transforms

APPENDIX B COMMENTS ON A THEOREM OF GODEMENT

APPENDIX C ON CERTAIN CLOSED SUBSETS OF A LOCALLY COMPACT ABELIAN GROUP

C.l The definition and some properties of $S_{p}-$ and

$$
C_{p} \text {-sets }
$$

C.2 Examples of $S_{p^{-}}$and $C_{p^{- \text {sets }}}$

C.3 The failure of certain closed sets to be $S_{p}$-sets 


\section{CHAPTER 0}

\section{INTRODUCTION AND PRELIMINARIES}

\subsection{Introduction and background to Bernstein's inequality}

In 1887 at St Petersburg the well-known chemist D.I. Mendeleev published a monograph on his investigations of the specific gravity of aqueous solutions. Aside from the contribution to the study of chemistry this work (see Mendeleev [1], Chapter 4) gave rise to an interesting mathematical problem with many applications in approximation theory and the study of singular integrals. Mendeleev proved that if a rational polynomial $P(x)$ of degree at most 2 is such that $|P(x)| \leq 1$ on $[-1,1]$ then $\left|P^{\prime}(x)\right| \leq 4$ on the same interval. Two years later Markov ([1]) proved the corresponding problem with polynomials of degree $n$; in this case the upper bound on the modulus of the derivative is $n^{2}$, equality holding if and only if $P(x)= \pm T_{n}(x)$ (where $T_{n}(x)=\cos (n \operatorname{arcos}(x))$ is the Chebyshev polynomial of the first kind of degree $n$.)

An extension of the above result into a Fourier analytic setting was given by S.N. Bernstein. In his paper on the approximation of continuous functions by polynomials (see Bernstein [1]) he proved the following inequality: if the trigonometric polynomial

$$
T_{n}(\theta)=\sum_{m=0}^{n}\left(a_{m} \cos (m \theta)+b_{m} \sin (m \theta)\right) \quad\left(a_{m}, b_{m} \in \mathrm{R}\right)
$$

does not exceed $I$ in modulus over $[-\pi, \pi]$, then

$$
\left|T_{n}^{\prime}(\theta)\right| \leq 2 n
$$

over the same interval. In the following twenty years this result was improved upon and generalised to give:

Bernstein's inequality. Let $T_{n}$ be a trigonometric polynomial, with 
complex coefficients, of degree at most $n$. Then for any $p \in[1, \infty]$

$$
\left\|T_{n}^{\prime}\right\|_{p} \leq n\left\|T_{n}\right\|_{p}
$$

There are various proofs of Bernstein's inequality in the case $p=\infty$. Perhaps the nicest and, from our point of view, the most useful was that of F. Riesz [1] who observed that for an appropriate choice of trigonometric polynomial $k_{n}$ of degree $n$ (independent of the choice of $T_{n}$ )

$$
T_{n}^{\prime}=k_{n} * T_{n}
$$

He thus reduced the problem to that of estimating the $L^{l}$-norm of $k_{n}$. By choosing

$$
k_{n}(x)=-2 n K_{n-1}(x) \sin (n x)
$$

Riesz obtained the inequality

$$
\left\|T_{n}^{\prime}\right\|_{\infty} \leq 2 n\|T\|_{\infty}
$$

here $K_{n-1}$ is the Féjer kernel of order $n-1$. To prove the more refined version of Bernstein's inequality he noted that (without affecting (0.0.1)) $\sin (n x)$ could be replaced by

$$
g(n x)=\sin (n x)-r^{2} \sin (3 n x)+r^{4} \sin (5 n x)-\ldots .
$$

The result then followed readily. Another proof was given by M. Riesz [1] who expressed the derivative in terms of the values of the polynomial at $2 n$ equidistant points. Zygmund [1] made use of both these proofs to extend the inequality to the case with $1 \leq p \leq \infty$.

The extension (in the case $p=\infty$ ) of Bernstein's inequality to the real line follows from a result of Bernstein [2] who proved that if $F(z)$ is an entire function of exponential type satisfying

$$
|F(z)|=O(\exp (\alpha x)) \text { and }|F(x)| \leq 1
$$

for all $x \in R$, where $\alpha \in R^{+}$, then

$$
\left|F^{\prime}(x)\right| \leq \alpha
$$


on $R$. All that is required now is the observation that a bounded function $f$ on $R$ is the restriction of an entire function of exponential type $\alpha \geq 0$ if and only if

$$
\Sigma(f) \subset[-\alpha, \alpha],
$$

$\Sigma(f)$ denoting the spectrum of $f$; this is just an extension of one of the Paley-Wiener theorems, see Kahane [1], p. 30. The case $1 \leq p<\infty$ was dealt with by Gosselin [1].

Other formulations of Bernstein's inequality (still involving functions of a real or complex variable) have been investigated over the past fifty years (for reasonably comprehensive surveys of these results, see Schaeffer [1] and Boas [1]). However in this thesis we take a different course and consider analogues of this inequality for pth-power integrable functions on arbitrary locally compact Hausdorff Abelian and compact Hausdorff groups. In view of the above results it seems natural to use (in place of trigonometric polynomials) the set of pth-power integrable functions with spectra contained in a given relatively compact set. Since, for a general group, the usual concept of the derivative is lacking we shall be examining inequalities of the form

$$
\|\|_{a} f-f\left\|_{p} \leq \omega(a)\right\| f \|_{p},
$$

where $\lim \omega(a)=0$ and $f \in L^{p}(G)$ with $\Sigma(f) \subset T$ (where $T$ is $a \rightarrow 0$

relatively compact). Thus the problem is effectively that of finding a joint mean modulus of continuity with exponent $p$ for

$$
\left\{f \in L^{p}(G): \Sigma(f) \subset T\right\} .
$$

In Chapter 1 we shall look at inequalities of the form $(0.0 .2)$ for various sets of functions; Chapter 2 will deal with a converse of this result; and in Chapter 3 we shall examine a version of Bemstein's inequality and its converse in a more classical setting with the left-hand side of $(0.0 .2)$ replaced by the derivative of $f$ along a one-parameter 
subgroup of $G$.

Most of the material in Chapters 1 and 2 has appeared in Bloom [1], [2] respectively; [1] also contains part of the "direct" result appearing in Chapter 3 .

\subsection{General references}

All of the analysis in this thesis will be carried out over locally compact Hausdorff topological groups; with few exceptions the groups will be Abelian and otherwise they will be compact.

We shall take Hewitt and Ross [1] to be our standard reference on topological groups. The symbols $R, C, T$ and $Z$ will be reserved for the additive groups of the real and complex numbers, the circle group, and the group of integers respectively.

For harmonic analysis our references will be any one of the books: Hewitt and Ross [1], Reiter [1] and Rudin [1]. We shall follow Hewitt and Ross [1] for integration theory.

Invariably our references for functional analysis and topology will be R.E. Edwards [1] and Bourbaki [1] respectively.

\subsection{Definitions and notation}

In the following list we collect together some of the frequently used basic definitions and notation; these will be standard throughout.

\section{2 .1 LOCALLY COMPACT GROUPS}

The symbol $G$ will always denote a locally compact Hausdorff group, and will possess typical elements $a, x, y, \ldots$. Unless stated otherwise $G$ will be assumed to be Abelian, its identity element being denoted by 0 . The dual group of $G$ will be denoted by $\Gamma$, and will possesstypical 
elements $\gamma, x, \ldots$.

\section{2 .2 HAAR MEASURE}

We shall denote by $\lambda, \theta$ Haar measures on $G, \Gamma$ respectively. These Haar measures will be assumed to be normalised so that Plancherel's theorem is valid. In the further case that one of $G, \Gamma$ is compact then its respective Haar measure will have total variation equal to 1 . Where no confusion will arise we abbreviate $\int_{G} f(x) d \lambda(x)$ by $\int_{G} f(x) d x$ or $\int_{G} f d \lambda$

\subsubsection{SPACES OF FUNCTIONS AND MEASURES}

We shall denote by $C(G)$ the space of bounded continuous functions on $G$, and write $C_{u}(G), C_{0}(G)$ and $C_{00}(G)$ for those subspaces of $C(G)$ comprising of functions which are uniformly continuous, which vanish at infinity, and which have compact support respectively. Each of these spaces will be equipped with the uniform norm.

$M_{b}(G)$ will denote the space of bounded complex Radon measures on $G$, and will be equipped with its usual Banach space topology (as the dual of $\left.C_{0}(G)\right)$; the norm of $\mu \in M_{b}(G)$ will be denoted by $\|\mu\|_{M}$. For the Dirac measure placed at $0 \in G$ we shall write $\varepsilon$.

When $p \in[1, \infty], L^{p}(G)$ will denote the usual normed Lebesgue space of equivalence classes of functions with norm $\|\cdot\|_{p}$. On occasions a function will be confounded with the equivalence class to which it belongs. We shall write " $f=g$ " to mean either "the class $f=$ the class $g$ " or "f $=g$ almost everywhere (or locally almost everywhere when $f, g \in L^{\infty}(G)$ )". It will be clear from the context what is meant in these cases. 
that is, $I^{\prime}=\infty, \infty^{\prime}=1$ and $p^{-1}+p^{\prime-1}=1$ for $p \in(1, \infty)$.

The characteristic function of a set $E$ will be written $\xi_{E}$.

\subsubsection{TRANSLATIONS AND REFLECTIONS}

For $a \in G$ we define the left $a$-translate of $f \in c^{G}$ by

$$
a^{f: x+f(-a+x) \quad(x \in G),}
$$

and of $\mu \in M_{b}(G)$ by

$$
a^{\mu(g)}=\mu\left(a^{g}\right)
$$

where $\mu(g)=\int_{G} g d \mu$. Right translates are defined similarly.

We also define the reflection of $f \in C^{G}$ by

$$
f_{v}: x \rightarrow f(-x)
$$

and of $\mu \in M_{b}(G)$ by

$$
\mu_{v}(g)=\mu\left(g_{v}\right)
$$

Finally we abbreviate $\bar{f}_{\vee}$ by $f^{\sim}$.

0.2.5 THE FOURIER TRANSFORM OF A FUNCTION IN $L^{p}(G)$

If $f \in L^{l}(G)$ we write $\hat{f}$ for the Fourier transform of $f$, defined by

$$
\hat{f}(\gamma)=\int_{G} f \bar{\gamma} d \lambda
$$

The subspace of $C_{0}(\Gamma)$ consisting of those functions which are the Fourier transforms of functions in $L^{l}(G)$ will be denoted by $A(\Gamma)$.

We shall give ' $\wedge$ ' a dual role; if $g \in C_{0}(\Gamma)$ is a Fourier transform then $\hat{g}$ will denote that function (or measure) on $G$ whose transform is $g$, in other words, the "inverse" transform of $g$. 
For $p \in(1, \infty]$ and $G$ Abelian we follow Gaudry [2] and define the Fourier transform of $f \in L^{p}(G)$ as the continuous linear form on $D(\Gamma)$ given by

$$
\hat{f}(g)=f\left(\hat{g}_{v}\right)
$$

(for the definition of $D(\Gamma) \subset C_{00} \cap A(\Gamma)$ see Gaudry [1], Section 2). It appears that when $p \in(1,2]$ the Fourier transform defined as above coincides with the usual Fourier transform; for the latter see Hewitt and Ross [1], Section 31. Note also that when $p=\infty, \hat{f}$ is actually a pseudomeasure on $\Gamma$.

Some properties of '^ ' can be found in Appendix A.

\subsubsection{THE SPECTRUM OF A FUNCTION IN $L^{p}(G), G$ ABELIAN}

Given $f \in L^{\infty}(G)$ we define its spectrum to be

$$
\Sigma(f)=\left\{\gamma \in \Gamma: g \in L^{l}(G) \text { and } g^{\sim} * f=0 \text { implies that } \hat{g}(\gamma)=0\right\} \text {. }
$$

This definition of the spectrum (due to R. Godement) can be extended to $f \in L^{P}(G) \quad(p \in[1, \infty])$ by defining

$$
\Sigma(f)=\underset{\phi \in C_{00}(G)}{U} \Sigma(\phi * f) .
$$

Alternatively, if $p \in(1, \infty], f \in L^{P}(G)$ and $\Omega \subset \Gamma$ is open, we say that $\hat{f}$ vanishes on $\Omega$ if

$$
\hat{f}(h)=0
$$

for all $h \in D(\Gamma)$ with $\operatorname{supp}(h) \subset \Omega$. The support of $\hat{f}$ (see Gaudry [1]) can then be defined as the complement (relative to $\Gamma$ ) of the largest open set on which $\hat{f}$ vanishes; and is written $[\hat{f}]$.

It is shown in Appendix A that if $p \in(1, \infty]$ and $f \in L^{p}(G)$ then

$$
\Sigma(f)=[\hat{f}] \text {, }
$$

and if $f \in L^{l}(G)$ then

$$
\Sigma(f)=\operatorname{supp}(\hat{f}) .
$$




\section{CHAPTER 1}

\section{BERNSTEIN'S INEQUALITY FOR LOCALLY COMPACT GROUPS}

\subsection{Introduction}

The basis of many of the results used in this chapter and indeed, throughout the thesis, is the exploitation of an idea successfully used by F. Riesz [l] who observed that for any trigonometric polynomial $f_{n}$ of degree at most $n$,

$$
f_{n}^{\prime}=k_{n} * f_{n}
$$

for some suitable trigonometric polynomial $k_{n}$ (depending only on $n$ ). Following this line we find that the problem of estimating the mean modulus of continuity with exponent $p$ of $f \in L^{p}(G)$ with $T=\Sigma(f)$ relatively compact is made easier by writing

$$
a^{f}-f=\left(a^{k}-k\right) * f
$$

for some suitable $k \in L^{l}(G)$ (independent of the choice of $f \in L^{p}(G)$ with $\Sigma(f) \subset T)$ and estimating the mean modulus of continuity with exponent $I$ of $k$.

To achieve this we shall frequently have recourse to a restricted version of Theorem 2.6.1 of Rudin [1], as stated in Appendix B.

\subsection{Estimates for $B_{\mathrm{T}}^{L}(a)$ on locally compact Abelian groups}

In general we shall be interested in some translation-invariant linear space $L(G) \subset L^{p}(G) \quad(p \in[1, \infty])$ with the property

$$
L^{\perp} * L(G) \subset L(G) \text {, }
$$

and having a norm $\|\cdot\|_{L}$ such that 


$$
\|g * f\|_{L} \leq\|g\|_{1}\|f\|_{L}
$$

for all $g \in L^{l}(G), f \in L(G)$.

The set of functions in $L(G)$ for which a Bernstein-type inequality will be obtained is the translation-invariant linear subspace $L_{T}(G)$, defined by

$$
L_{\Upsilon}(G)=\{f \in L(G): \Sigma(f) \subset \Upsilon\},
$$

where $T \subset \Gamma$ is relatively compact. For each $a \in G$ we put

$$
\beta_{T}^{L}(a)=\sup \left\{\left\|_{\alpha} f-f\right\|_{L}: f \in L_{T}(G),\|f\|_{L} \leq I\right\}
$$

and

$$
\omega_{\Upsilon}(a)=\sup \{|\gamma(a)-1|: \gamma \in \Upsilon\},
$$

where $\omega_{\Upsilon}(a)$ is defined to be zero if $T$ is empty. It is easily shown that for $\mathrm{T}, \mathrm{T}_{1}, \mathrm{~T}_{2} \subset \Gamma$

$$
\begin{gathered}
\omega_{-T}=\omega_{T}, \\
\omega_{T_{1}+T_{2}} \leq \omega_{T_{1}}+\omega_{T_{2}},
\end{gathered}
$$

and

$$
\omega_{T_{1} \cup T_{2}} \leq \max \left\{\omega_{T_{1}}, \omega_{T_{2}}\right\}
$$

Furthermore, if $T \subset \Gamma$ is relatively compact, then the definition of the topology of $\Gamma$ (as the dual of $G$ ) gives immediately

$$
\lim _{a \rightarrow 0} \omega_{\mathrm{T}}(a)=0 \text {. }
$$

Given a relatively compact set $T \subset \Gamma$ we shall (because of (1.1.3)) examine estimates for $\beta_{\mathrm{T}}^{L}(\alpha)$ of the form

$$
\beta_{T}^{L}(a)=O\left(\omega_{T},(a)\right)
$$

where $T^{\prime}$ is some relatively compact subset of $\Gamma$. As a preliminary result we require: 
1.1.1 LEMMA. Let $T$ be a relatively compact subset of $\Gamma$ and suppose $k, l \in L^{l}(G)$ are such that $\hat{k}=1$ and $\hat{\imath}=0$ on a neighbourhood of $T$. Then

$$
\beta_{T}^{L}(a) \leq\left\|a^{k}-k-z\right\|_{1}
$$

Proof. We show initially that if $k, l$ satisfy the hypotheses of the lemma then, for $f \in L_{\gamma}(G)$,

$$
\tau * f=0 \text { and } k * f=f \text {. }
$$

For this it suffices to show that $(1.1 .4)$ holds pointwise locally almost everywhere (since a function in $L^{P}(G) \quad(p \neq \infty)$ which vanishes locally almost everywhere vanishes almost everywhere).

By A.1.2 and A.2.I

$$
\Sigma(2 * f)=\square,
$$

whence it follows (see Reiter [1], Chapter 7, 1.3 (i)) that

$$
\phi * \tau * f=0
$$

for every $\phi \in C_{00}(G)$, and hence $Z * f=0$ locally almost everywhere. Now, if $\phi \in C_{00}(G)$ then $(k * \phi-\phi)^{\wedge}$ vanishes on a neighbourhood of $\mathrm{T}$ and, by what has already been established,

$$
(k * \phi-\phi) * f=0 \quad \text { l.a.e. . }
$$

Since $\phi \in C_{00}(G)$ was chosen arbitrarily

$$
k * f=f \text { l.a.e., }
$$

proving (1.1.4).

Using (1.1.4) and the fact that $\Sigma\left({ }_{a} f\right)=\Sigma(f)$ we have

$$
\begin{aligned}
a^{f-f} & =k *\left(a_{a} f-f\right)-z * f \\
& =\left(a^{k}-k-2\right) * f
\end{aligned}
$$

and, by (1.1.1) and (1.1.2),

$$
\left\|a_{a}^{f}-f\right\|_{L} \leq\left\|a_{a}-k-\tau\right\|_{I}\|f\|_{L},
$$

from which the result follows. 
With a suitable choice of $k, l \in L^{l}(G)$ satisfying the conditions of Lemma 1.1.1 we can obtain an estimate for $\beta_{T}^{L}(a)$.

1.1.2 THEOREM. Let $T, \nabla$ be relatively compact subsets of $\Gamma$, where $\nabla$ is non-void and open. Then

$$
\beta_{T}^{L}(\alpha) \leq\left(\frac{\theta(T+\nabla-\nabla)}{\theta(\nabla)}\right)^{\frac{1}{2}}\left(\omega_{T+\nabla-\nabla}(\alpha)+\omega_{\nabla}(\alpha)\right)
$$

Proof. Using the method of the proof of Theorem B.I.I we choose $g, h \in L^{2}(G)$ such that $\hat{g}=\xi_{\nabla}, \hat{h}=\xi_{\Upsilon+\nabla-\nabla}$ and put

$$
k=\theta(\nabla)^{-1} g h \text {. }
$$

Then $k \in L^{l}(G), \hat{k}=1$ on $T+\nabla$ and

$$
\begin{aligned}
\left\|_{a} k-k\right\|_{1} & =\theta(\nabla)^{-1}\left\|\left(a_{a} h-h\right) g+(a g-g)\left(a_{a} h\right)\right\|_{1} \\
& \leq \theta(\nabla)^{-1}\left(\left\|{ }_{a} h-h\right\|_{2}\|g\|_{2}+\left\|a_{a} g-g\right\|_{2}\|h\|_{2}\right) \\
& \leq \theta(\nabla)^{-1}\left(\omega_{1}+\nabla-\nabla\right. \\
& \left.\leq(a)\|h\|_{2}\left\|_{2}\right\|_{2}+\omega_{\nabla}(a)\|g\|_{2}\|h\|_{2}\right) \\
& \leq\left(\frac{\theta(T+\nabla-\nabla)}{\theta(\nabla)}\right)^{\frac{1}{2}}\left(\omega_{T+\nabla-\nabla}(a)+\omega_{\nabla}(a)\right),
\end{aligned}
$$

the final two steps using Plancherel's theorem. Now apply Lemma 1.1.1. //

1.1.3 COROLLARY. Suppose the hypotheses of Theorem 1.1 .2 are satisfied and $0 \in \nabla$. Then

$$
\beta_{\Upsilon}^{L}(a) \leq 3\left(\frac{\theta(T+\nabla-\nabla)}{\theta(\nabla)}\right)^{\frac{1}{2}} \omega_{T+\nabla-\nabla}(a)
$$

Proof. We can assume that $T$ is non-empty. Then, choosing $\gamma \in T$, we have

$$
\begin{aligned}
\omega_{\nabla}(a) & \leq \omega_{-\gamma+\Upsilon+\nabla-\nabla}(a) \\
& \leq \omega_{-\gamma}(a)+\omega_{\Upsilon+\nabla-\nabla}(a) \\
& \leq 2 \omega_{\Upsilon+\nabla-\nabla}(a),
\end{aligned}
$$


For certain relatively compact sets $T \subset \Gamma$ we can obtain estimates of the form

$$
\beta_{T}^{L}(\alpha)=O\left(\omega_{T}(\alpha)\right)
$$

As would be expected $(1.1 .5)$ holds for relatively compact sets $T$ which, in a certain sense, generate open sets. In fact we have:

1.1.4 THEOREM. Let $T \subset \Gamma$ be relatively compact and suppose there exists a positive integer $n$ for which $n T$ has non-void interior. Then

$$
\beta_{T}^{L}(a) \leq \kappa \omega_{T}(a)
$$

where $k=k(T)$.

Proof. Suppose $T, n$ satisfy the hypothesis of the theorem and choose any $\gamma \in \operatorname{int}(n T)$. Then

$$
T \subset T-\gamma+\operatorname{int}(n T)
$$

We can find a relatively compact open neighbourhood $\nabla$ of zero in $\Gamma$ such that

$$
T+\nabla-\nabla \subset T-\gamma+\operatorname{int}(n T),
$$

and we have

$$
\begin{aligned}
\omega_{\Upsilon+\nabla-\nabla}(\alpha) & \leq \omega_{\Upsilon}(\alpha)+\omega_{-\gamma}(\alpha)+\omega_{\operatorname{int}(n T)}(\alpha) \\
& \leq(2 n+1) \omega_{\Upsilon}(\alpha) .
\end{aligned}
$$

The result now follows from corollary 1.1.3. //

1.1.5 REMARK. The hypothesis of Theorem 1.1 .4 is satisfied whenever $\theta(T)>0 \quad($ see Hewitt and Ross [1], (20.17))

1.1.6 REMARK. All of the results of Section 1.1 are slightly simplified when $T$ is a set of spectral synthesis ( $S$-set). In this case we just replace "on a neighbourhood of $T$ " and " $T+\nabla$ " by "on $T$ " and "T" respectively. 
1.2 Estimates for $\beta_{\mathrm{T}}^{L}(a)$ when $L$ is a *-module over a weighted $L^{1}$-space

It may happen that the translation-invariant linear subspace

$L(G) \subset L^{p}(G) \quad(p \in[1, \infty])$ satisfies only the possibly weaker condition:

$$
L_{w}^{\perp} * L(G) \subset L(G)
$$

and there is a norm. $\|\cdot\|_{L}^{\prime}$ on $L$ such that for all $g \in L_{w}^{I}(G)$ and $f \in L(G)$,

$$
\|g * f\|_{L}^{\prime} \leq\|g\|_{1, \omega}\|f\|_{L}^{\prime}
$$

$w$ is a weight function, that is, a non-negative locally bounded measurable function satisfying

$$
w(x) \geq 1 ; \quad w(x+y) \leq w(x) w(y)
$$

for all $x, y \in G$, and

$$
L_{\omega}^{1}(G)=\left\{g \in L^{I}(G):\|g\|_{1, \omega}=\int_{G}|g| w d \lambda<\infty\right\} .
$$

Under these conditions the proof of Lemma 1.1.1 carries over quite easily to give:

\subsubsection{LEMMA. Let $T$ be a relatively compact subset of $\Gamma$ and} suppose $k, l \in L_{w}^{l}(G)$ are such that $\hat{k}=1$ and $\hat{\imath}=0$ on a neighbourhood of $T$. Then

$$
\beta_{\mathrm{T}}^{L}(\alpha) \leq\left\|a^{k}-k-\tau\right\|_{1, \omega} \cdot
$$

When we attempt to follow the proof of Theorem 1.1.2 to obtain an analogous result, we run into the problem of determining whether gw and $h w$ belong to $L^{2}(G)$. This problem can be handled if we assume that the weight function $w$ satisfies the so-called condition of Beurling-Domar, namely 


$$
\sum_{n=1}^{\infty} n^{-2} \log (w(n x))<\infty
$$

for all $x \in G$; see Domar [1], Theorem 2.11. This theorem of Domar (using his terminology) then states that $L_{w}^{l}(G)^{\wedge}$ is an algebra of type $F$ and hence, see Domar [1], Lemma 1.24, $L_{w}^{1}(G)^{\wedge}$ is a regular normed algebra (in the sense of G. Šilov; see Godement [1], p. 123 (i)). We have:

1.2.2 THEOREM. Choose $T, \nabla \subset \Gamma$ to be relatively compact sets, each containing zero, and such that $\nabla$ is symmetric and open. If $w$ is a weight function satisfying $(1.2 .1)$ and $V$ is any relatively compact neighbourhood of zero in $G$ then

$$
\beta_{T}^{L}(a) \leq \kappa \omega_{T+3 \nabla^{(a)}}
$$

where $k=k(T, \nabla, V, w)$.

Proof. It is clear that $w^{2}$ is a weight function satisfying $(1.2 .1)$. Hence we can appeal to Domar [1] (Theorem 2.11 and Lemma 1.24) to deduce the existence of $g, h \in L_{w^{2}}^{1} \cap C_{0}(G)$ such that

$$
\xi_{\{0\}} \leq \hat{g} \leq \xi_{\nabla}
$$

and

$$
\xi_{\Upsilon+2 \nabla} \leq \hat{h} \leq \xi_{\Upsilon+3 \nabla}
$$

Now both $g w$ and $h w$ are in $L^{2}(G)$. Indeed, since $g \in C_{0}(G)$, we have the existence of a compact set $C \subset G$ such that $|g(x)| \leq 1$ for all $x \in G \backslash C$ and, using the property of $w^{2}$ being locally bounded, it follows that

$$
\begin{aligned}
\int_{G}|g|^{2} w^{2} d \lambda & =\int_{C}|g|^{2} w^{2} d \lambda+\int_{G \backslash C}|g|^{2} \omega^{2} d \lambda \\
& \leq \int_{C}|g|^{2} w^{2} d \lambda+\int_{G \backslash C}|g|^{2} d \lambda \\
& <\infty
\end{aligned}
$$


(and similarly for hw).

Putting

$$
k=\alpha^{-1} g h \text {, }
$$

where

$$
\alpha=\int_{\nabla} \hat{g} d \theta
$$

we see that $k \in L_{w}^{l}(G)$ and, for any $\gamma \in \Gamma$,

$$
\begin{aligned}
\hat{k}(\gamma) & =\alpha^{-1} \int_{\Gamma} \hat{g}(x) \hat{h}(\gamma-x) d x \\
& =\left\{\int_{\nabla} \hat{g} d \theta\right)^{-1} \int_{\nabla} \hat{g}(x) \hat{h}(\gamma-x) d x
\end{aligned}
$$

If $\gamma \in T+\nabla$ then $\gamma-x \in T+2 \nabla$ and, from $(1.2 .3), \hat{h}(\gamma-\chi)=1$. Consequently $\hat{k}=1$ on $\Upsilon+\nabla$. Furthermore

$$
\begin{aligned}
& \left\|a^{k}-k\right\|_{1, w} \leq \alpha^{-1}\left(\left\|\left(a^{g}-g\right)\left(a^{h}\right)\right\|_{1, w}+\left\|\left(a^{h}-h\right) g\right\|_{1, w}\right) \\
& \leq \alpha^{-1}\left(\left\|_{\alpha^{g}} g-g\right\|_{2}\left\|\left(a^{h}\right) w\right\|_{2}+\left\|\alpha_{a} h-h\right\|_{2}\|g w\|_{2}\right) \\
& \left.\leq \alpha^{-1}\left(\| C_{a} h\right) w\left\|_{2} \omega_{\nabla}(a)\right\| g\left\|_{2}+\right\| g \omega\left\|_{2} \omega_{\mathrm{T}+3 \nabla}(\alpha)\right\| h \|_{2}\right) \\
& \leq \kappa^{\prime} \omega_{\mathrm{T}+3 \nabla}(\alpha)
\end{aligned}
$$

where (using $(1.2 .2)$ and $(1.2 .3)$ )

$$
\kappa^{\prime}=\alpha^{-1}\left[w(\alpha)\|h w\|_{2} \theta(\nabla)^{\frac{1}{2}}+\|g w\|_{2} \theta(\Upsilon+3 \nabla)^{\frac{1}{2}}\right\} \text {. }
$$

Finally, since $w$ is locally bounded and $V$ is relatively compact, there is a constant $K^{\prime \prime}$ such that for all $a \in V$

$$
w(a) \leq \kappa^{\prime \prime}
$$

The result now follows from Lemma 1.2.1. //

1.2.3 COROLLARY. Let $T \subset \Gamma$ be a relatively compact set containing zero and suppose there exists a positive integer $n$ for which $n T$ has nonvoid interior. Then, if $w, V$ satisfy the conditions of Theorem 1.2.2, we have 


$$
\beta_{T}^{L}(\alpha) \leq k \omega_{T}(\alpha)
$$

where $k=k(T, w, V)$.

Proof. See the proof of Theorem 1.1.4. $1 /$

\subsection{Estimates for $\beta_{T}^{p}(a)$ on locally compact Abelian groups}

We know from Lemma 1.1.1 and Remark 1.1 .6 that if $T \subset \Gamma$ is a relatively compact $S$-set then

$\beta_{\mathrm{T}}^{L}(a) \leq \inf \left\{\|\|_{a} k-k-\tau \|_{1}: k, \tau \in L^{1}(G), \hat{k}=1, \hat{\imath}=0\right.$ on $\left.\mathrm{T}\right\}$.

It is natural to ask when equality occurs in (1.3.1). Section 1.3 is concerned with this and related questions when $L(G)=L^{P}(G) \quad(p \in[1, \infty])$; in this case we shall abbreviate $\beta_{T}^{L^{p}}(a)$ to $\beta_{T}^{p}(\alpha)$.

1.3.1 THEOREM. Suppose $T \subset \Gamma$ is a compact $S$-set such that

$$
T=\left(U\left\{\Sigma(f): f \in C_{0, T}(G)\right\}\right\}^{-} \text {. }
$$

Then

$\beta_{T}^{\infty}(a)=\inf \left\{\left\|_{a} k-k-l\right\|_{1}: k, l \in L^{l}(G), \hat{k}=1, \hat{\imath}=0\right.$ on $\left.T\right\} \quad(a \in G)$.

Proof. It is clear that $C_{0, T}(G)$ is non-void. Consider the complexvalued map

$$
T: C_{0, T}(G) \rightarrow C,
$$

defined by

$$
T(f)=f(-a)-f(0)
$$

Since $T$ is linear and bounded the Hahn-Banach theorem ensures that it can be extended to a bounded linear functional $T^{\prime}$ on $C_{0}(G)$ such that

$$
\left\|T^{\prime}\right\| \leq\|T\|
$$

Using the Riesz representation theorem we can assert the existence of $\mu \in M_{b}(G)$ such that for all $f \in C_{0}(G)$ 


$$
\begin{aligned}
T^{\prime}(f) & =\int_{G} f_{v} d \mu \\
& =\mu * f(0) .
\end{aligned}
$$

Clearly

$$
\begin{aligned}
\|\mu\|_{M} & =\left\|T^{\prime}\right\| \\
& \leq\|T\| \\
& =\sup \left\{|f(-a)-f(0)|: f \in C_{0, T}(G),\|f\|_{\infty} \leq 1\right\} \\
& \leq \sup \left\{\left\|_{a} f-f\right\|_{\infty}: f \in C_{0, T}(G),\|f\|_{\infty} \leq 1\right\} \\
& \leq \sup _{a}\left\{\left\|_{\alpha} f-f\right\|_{\infty}: f \in L_{T}^{\infty}(G),\|f\|_{\infty} \leq 1\right\}, \\
& =\beta_{T}^{\infty}(a) .
\end{aligned}
$$

Given $f \in C_{0, T}(G)$ and $x \in G$ we have, using (1.3.3) and the fact that $\Sigma\left({ }_{-x} f\right)=\Sigma(f)$,

$$
\begin{aligned}
\left(\int_{-x} f\right)(-a)-\left(_{-x} f\right)(0) & =T\left(_{-x} f\right) \\
& =\mu *\left(\int_{-x} f\right)(0) \\
& =(-x(\mu * f))(0),
\end{aligned}
$$

or equivalently,

$$
f(x-a)-f(x)=\mu * f(x) .
$$

Hence, for every $f \in C_{0, T}(G)$,

$$
a^{f}-f=\mu * f
$$

Now choose any $\varepsilon>0$. There exists $g \in L^{l}(G)$ such that $\hat{g}=1$ on $\mathrm{T}$ and $\|g\|_{1}<1+\varepsilon$ (see Hewitt and Ross [1], (31.37)). Putting $h=\mu * g$ we see that $\hat{h}=\hat{\mu}$ on $T$ and, since $T$ is assumed to be an $S$-set, it follows that for all $f \in C_{0, T}(G)$

$$
\begin{aligned}
h * f & =\mu * f \\
& =a f-f .
\end{aligned}
$$


Furthermore, by $(1.3 .4)$ and the choice of $g$,

$$
\|h\|_{1} \leq(1+\varepsilon) \beta_{T}^{\infty}(a)
$$

Let $k \in L^{l}(G)$ be such that $\hat{k}=1$ on $T$. We want to show that $\left(h-a^{k}+k\right)^{\wedge}$ vanishes on $T$. For any $f \in C_{0, T}(G)$ we have, using (1.3.5) and again the fact that $T$ is an $S$-set,

$$
\begin{aligned}
\left(h-a^{k+k}\right) * f & =a^{f}-f-a^{k * f+k * f} \\
& =0 .
\end{aligned}
$$

Hence $\left(h-a^{k+k}\right)^{\wedge}$ vanishes on $\Sigma(f)$ and, from (1.3.2) and the continuity of $\left(h-a^{k+k}\right)^{\wedge}$, it must vanish on $T$.

Put $-l=h-a^{k+k}$. Then $l \in L^{l}(G)$ and $\hat{\imath}=0$ on $T$. Furthermore, by $(1.3 .6)$,

$$
\begin{aligned}
\| a_{a}^{k-k-z \|_{I}} & =\|h\|_{I} \\
& \leq(1+\varepsilon) B_{T}^{\infty}(a)
\end{aligned}
$$

and thus

$$
\inf \left\{\|\|_{\alpha}-k-\tau \|_{1}: k, \tau \in L^{l}(G), \hat{k}=1, \hat{\imath}=0 \text { on } T\right\} \leq(1+\varepsilon) \beta_{T}^{\infty}(\alpha) \text {. }
$$

Since $\varepsilon>0$ was chosen arbitrarily

$$
\inf \left\{\|\|_{a} k-k-z \|_{1}: k, z \in L^{I}(G), \hat{k}=1, \hat{\imath}=0 \text { on } T\right\} \leq \beta_{T}^{\infty}(a)
$$

which, combined with (1.3.1), gives the desired result.

$$
\text { /1 }
$$

1.3.2 COROLLARY. Suppose $T \subset \Gamma$ is a compact $S$-set which is the closure of its interior. Then

$$
\beta_{T}^{\infty}(\alpha)=\inf \left\{\|\|_{\alpha} k-k-l \|_{1}: k, l \in L^{l}(G), \hat{k}=1, \hat{\imath}=0 \text { on } \Upsilon\right\} \quad(a \in G) \text {. }
$$

Proof. In view of Theorem 1.3 .1 all we have to prove is that $T$ satisfies (1.3.2). Furthermore we can assume that $T$ is non-void.

Let $\gamma \in \operatorname{int}(T)$. Then (see B.I.I) we can find $f_{\gamma} \in L^{I} \cap C_{0, \operatorname{int}(T)}(G)$ 
such that $\hat{f}_{\gamma}(\gamma)=1$. Hence $\gamma \in \Sigma\left(f_{\gamma}\right)$, whence it follows that

$$
\operatorname{int}(T) \subset U\left\{\Sigma(f): f \in C_{0, T}(G)\right\}
$$

Since $T=\operatorname{int}(T)^{-},(1.3 .2)$ must hold for $T . \quad / /$

Results analogous to Theorem 1.3.I for the quantity $\beta_{T}^{p}(a)$

$(p \in[l, \infty))$ take a slightly different form. If we define

$$
B_{T}^{p^{\prime}}(G)=\left\{z \in L^{p^{\prime}}(G): \tau * f=0 \text { for all } f \in L_{T}^{p}(G)\right\} \text {, }
$$

then we have (note that $\Upsilon$ need not be relatively compact):

1.3.3 THEOREM. Let $T \subset \Gamma$. Then, for $p \in[1, \infty)$,

$$
\beta_{T}^{p}(a)=\sup _{\|\phi\|_{p^{\prime}} \leq 1} \inf _{z \in B_{T}^{p^{\prime}}(G)} \| a_{a^{\phi}-\phi-z \|_{p^{\prime}}}
$$

Proof. For any $f \in L_{T}^{p}(G), \phi \in L^{p^{\prime}}(G)$ and $l \in B_{T}^{p^{\prime}}(G)$ we have

$$
\begin{aligned}
\phi *\left(a^{f}-f\right) & =\phi * \alpha^{f}-\phi * f-Z * f \\
& =\left(a^{\phi}-\phi-l\right) * f,
\end{aligned}
$$

and hence

$$
\left|\phi *\left({ }_{a} f-f\right)(0)\right| \leq\|\|_{a}^{\phi}-\phi-z\left\|_{p},\right\| f \|_{p} .
$$

Since this holds for every $\phi \in L^{p^{\prime}}(G)$ and $l \in B_{T}^{p^{\prime}}(G)$ it follows that

$$
\left|\phi *\left({ }_{\alpha} f-f\right)(0)\right| \leq \inf _{z \in B_{T}^{p^{\prime}}(G)}\left\|_{\alpha} \phi-\phi-z\right\|_{p},\|f\|_{p}
$$

and, by the converse to Hölder's inequality,

$$
\begin{aligned}
\|f-f\|_{p} & =\sup _{\|\phi\|_{p^{\prime}} \leq I}\left|\phi *\left(a_{a} f-f\right)(0)\right| \\
& \leq \sup _{\|\phi\|_{p^{\prime}} \leq I} \inf _{\in B_{T}^{p^{\prime}}(G)}\|\|_{\alpha} \phi-\phi-\tau\left\|_{p^{\prime}},\right\| f \|_{p}
\end{aligned}
$$

or, equivalently,

$$
\beta_{T}^{p}(a) \leq \sup _{\|\phi\|_{p^{\prime}} \leq 1} \inf _{Z \in B_{T}^{p^{\prime}}(G)}\left\|_{a^{\phi}} \phi-\phi-Z\right\|_{p^{\prime}} \cdot
$$

To prove the reverse inequality we follow the line of the proof of Theorem 1.3.1 and consider the complex-valued map 


$$
T_{\phi}: L_{T}^{p}(G) \rightarrow C,
$$

defined by

$$
T_{\phi}(f)=\phi *\left({ }_{\alpha} f-f\right)(0),
$$

where $\phi \in L^{p^{\prime}}(G)$ with. $\|\phi\|_{p^{\prime}} \leq 1$. It is clear that $T_{\phi}$ is linear and, since

$$
\begin{aligned}
\left\|T_{\phi}\right\| & =\sup \left\{\left|\phi *\left({ }_{a} f-f\right)(0)\right|: f \in L_{T}^{p}(G),\|f\|_{p} \leq 1\right\} \\
& \leq \sup \left\{\left|f *\left({ }_{a} \phi-\phi\right)(0)\right|: f \in L^{p}(G),\|f\|_{p} \leq 1\right\} \\
& =\|\|_{a} \phi-\phi \|_{p^{\prime}} \\
& \leq 2
\end{aligned}
$$

we see that $T_{\phi}$ is bounded. By the Hahn-Banach theorem $T_{\phi}$ can be extended to a bounded linear functional $T_{\phi}^{\prime}$ on $L^{p}(G)$ such that

$$
\left\|T_{\phi}^{\prime}\right\| \leq\left\|T_{\phi}\right\| \text {. }
$$

It follows from Hewitt and Ross [1], (12.18) that there exists $h \in L^{p^{\prime}}(G)$ such that for all $f \in L^{p}(G)$,

$$
T_{\phi}^{\prime}(f)=\int_{G} f_{\sim} h d \lambda .
$$

Furthermore

$$
\begin{aligned}
\|h\|_{p^{\prime}} & =\left\|T_{\phi}^{\prime}\right\| \\
& \leq\left\|T_{\phi}\right\| \\
& \leq \sup \left\{\left\|_{a} f-f\right\|_{p}: f \in L_{T}^{p}(G),\|f\|_{p} \leq 1\right\} \\
& =\beta_{T}^{p}(a) .
\end{aligned}
$$

Now let $f \in L_{T}^{p}(G)$. Then, for any $x \in G$, we must have ${ }_{-2}^{f} f \in L_{\Upsilon}^{p}(G)$ 


$$
\begin{aligned}
\phi *\left(a_{a} f-f\right)(x) & =\phi *\left(_{a}(-x f)-x_{-x} f\right)(0) \\
& =T_{\phi}(-x f) \\
& =\int_{G}(-x f)_{\checkmark} h d \lambda \\
& =h * f(x),
\end{aligned}
$$

that is,

$$
\left(h-a^{\phi}+\phi\right) * f=0 \text {. }
$$

Since $f \in L_{T}^{p}(G)$ was chosen arbitrarily

$$
\tau=h-a^{\phi}+\phi \in B_{T}^{p^{\prime}}(G)
$$

and, from (1.3.8),

$$
\begin{aligned}
\left\|a^{\phi}-\phi-\tau\right\|_{p^{\prime}} & =\|h\|_{p^{\prime}} \\
& \leq \beta_{T}^{p}(a) .
\end{aligned}
$$

Hence

$$
\inf _{Z \in B_{\Upsilon}^{p^{\prime}}(G)}\|\|_{a^{\phi}}-\phi-z \|_{p^{\prime}} \leq \beta_{T}^{p}(a)
$$

and, since $\phi \in L^{p^{\prime}}(G)$ with. $\|\phi\|_{p^{\prime}} \leq 1$ was chosen arbitrarily,

$$
\sup _{\|\phi\|_{p^{\prime}} \leq 1} \inf _{\tau \in B_{T}^{p^{\prime}}}\left\|_{\alpha^{\phi}} \phi-\phi-Z\right\|_{p^{\prime}} \leq \beta_{T}^{p}(\alpha) .
$$

The result now follows using (1.3.7). //

With slight modifications we can obtain results similar to those in $1.3 .1-1.3 .3$ for the quantity

$$
\alpha \frac{p}{T}(a)=\sup \left\{\left\|_{\alpha} f-f\right\|_{p}: f \in L^{I} \cap L_{T}^{p}(G),\|f\|_{p} \leq I\right\} \text {. }
$$

Under the conditions of Theorem 1.3.1 (with $C_{0, T}(G)$ replaced by $\left.L^{I} \cap C_{0, T}(G)\right)$ it is easy to prove that 


$$
\alpha_{T}^{\infty}(\alpha)=\beta_{T}^{\infty}(\alpha)
$$

it is clear from the proof of Corollary 1.3 .2 that this is true when $T$ is a compact $S$-set which is the closure of its interior. Furthermore Theorem 1.3.3 holds with $\beta_{T}^{p}(a)$ replaced by $\alpha_{T}^{p}(a) \quad(p \in[1, \infty))$ and $B_{T}^{p^{\prime}}(G)$ replaced by

$$
A_{\Upsilon}^{p^{\prime}}(G)=\left\{l \in L^{p^{\prime}}(G): l * f=0 \text { for all } f \in L^{I} \cap L_{T}^{p}(G)\right\} \text {. }
$$

In general it seems difficult to relate the sets $A_{T}^{p^{\prime}}(G)$ and $B_{T}^{p^{\prime}}(G)$ to sets of the form $L_{\Delta}^{p^{\prime}}(G)$, where $\Delta \subset \Gamma$. However when $\operatorname{int}(T)^{c}$ is an $S_{p}$-set (see Definition C.I.I) we do have the following result:

1.3.4 THEOREM. Let $T \subset \Gamma$ and $p \in[1, \infty)$. Then the following conditions are equivalent:

(a) $\operatorname{int}(T)^{c}$ is an $S_{p}$-set; $^{\text {set }}$

(b) $A_{T}^{p^{\prime}}(G)=\left\{\tau \in L^{p^{\prime}}(G): \hat{\imath}=0\right.$ on $\left.\operatorname{int}(T)\right\}$.

Proof. $(a) \Rightarrow(b)$. Suppose that int $(T)^{c}$ is an $S_{p}$-set and choose $l \in L^{P^{\prime}}(G)$ with $\hat{\imath}=0$ on $\operatorname{int}(T)$. For any $f \in L^{I} \cap L_{T}^{p}(G)$ we see that $\hat{f}=0$ on $\operatorname{int}(T)^{c}$ and hence, given $\varepsilon>0$, there exists $g \in L^{\perp} \cap L^{p}(G)$ such that $\hat{g}=0$ on a neighbourhood of $\operatorname{int}(T)^{c}$ and

$$
\|Z\|_{p},\|f-g\|_{p}<\varepsilon \text {. }
$$

Now $\operatorname{supp}(\hat{g}) \subset \operatorname{int}(\Upsilon)$, and so

$$
z * g=0 \text {. }
$$

Thus

$$
\begin{aligned}
\|Z * f\|_{\infty} & =\|Z *(f-g)\|_{\infty} \\
& \leq\|Z\|_{p},\|f-g\|_{p} \\
& <\varepsilon
\end{aligned}
$$


and, since $\varepsilon>0$ was chosen arbitrarily,

$$
\tau * f=0 \text {, }
$$

that is, $\quad l \in A_{T}^{p^{\prime}}(G)$.

To show the reverse inclusion suppose $l \in L^{P^{\prime}}(G)$ and $z * f=0$ for every $f \in L^{I} \cap L_{T}^{p}(G)$. If $\operatorname{int}(T)=\square$ there is nothing to prove. Otherwise choose $\gamma \in \operatorname{int}(T)$ and $\Omega_{\gamma}$, a relatively compact open neighbourhood of $\gamma$, such that

$$
\Omega_{\gamma} \subset \Omega_{\gamma}^{-} \subset \operatorname{int}(T)
$$

We can find $f_{\gamma} \in L^{I} \cap C_{0, \operatorname{int}(T)}(G)$ such that $\hat{f}_{\gamma}=1$ on $\Omega_{\gamma}^{-}$. Then, since $\hat{\imath} \hat{f}=0, \hat{\imath}$ must vanish on $\Omega_{\gamma}$ and hence (see Gaudry [1], Lemma 2.13) on

$$
\underset{\gamma \in \operatorname{int}(T)}{U}{ }^{\Omega} \gamma=\operatorname{int}(T)
$$

$(b) \Rightarrow(a)$. Suppose to the contrary that $\operatorname{int}(T)^{C}$ is not an $S_{p}$-set. Then there exists $f \in L^{I} \cap L^{P}(G)$ such that $\hat{f}=0$ on $\operatorname{int}(T)^{c}$ and $f k w$, the $L^{p}$-closure of

$\left\{g \in L^{1} \cap L^{p}(G): \hat{g}\right.$ vanishes on a neighbourhood of $\left.\operatorname{int}(T)^{c}\right\}$. Using the Hahn-Banach theorem we can find a bounded linear functional $T$ on $L^{p}(G)$ such that $T(W)=\{0\}$ and $T(f)=1$. By Hewitt and Ross [1], (12.18), there exists $l \in L^{p^{\prime}}(G)$ such that for all $g \in L^{p}(G)$

$$
\begin{aligned}
T(g) & =\int_{G} g Z d \lambda \\
& =Z_{\checkmark} * g(0) .
\end{aligned}
$$

Since $T(W)=\{0\}$ it follows that $\hat{\imath}$ vanishes on $\operatorname{int}(T)$ and, by $(b)$,

$$
\text { Z*f }=0 \text {, }
$$

contrary to assumption. // 
1.3.5 COROLLARY. Let $T \subset \Gamma$ and $p \in[2, \infty)$. Then

$$
A_{T}^{p^{\prime}}(G)=\left\{l \in L^{p^{\prime}}(G): \hat{\imath}=0 \text { on } \operatorname{int}(T)\right\} \text {. }
$$

Proof. This follows from Theorem 1.3 .4 and the fact that for $p \geq 2$ every closed subset of $\Gamma$ is an $S_{p}$-set; see Theorem C.2.1. //

\subsection{Further estimates for $\beta_{\Upsilon}^{\infty}(a)$ on locally compact Abelian groups}

Initially we give a result which shows that any estimate for $\beta_{T}^{\infty}(a)$

will apply equally well to $\beta_{\Upsilon}^{L}(a)$ when $L$ is a member of a certain family of function spaces (which includes the $L^{p}$-spaces).

1.4.1 THEOREM. Let $T \subset \Gamma$ and suppose $L(G)$ satisfies (1.1.1), (1.1.2) and has the additional property that there exists $Q \subset \mathcal{C}_{00}(G)$ such that for all $f \in L(G)$,

$$
\|f\|_{L}=\sup \left\{\|\phi * f\|_{\infty}: \phi \in 2\right\} .
$$

Then

$$
\beta_{\Upsilon}^{L}(a) \leq \beta_{\Upsilon}^{\infty}(a)
$$

Proof. Let $\phi \in 2$ and $f \in L_{\Upsilon}(G)$. Then $\phi * f \in L_{\Upsilon}^{\infty}(G)$ and, using $(1.4 .1)$,

$$
\begin{aligned}
\left.\| \phi *()_{a} f-f\right) \|_{\infty} & =\left\|_{a}(\phi * f)-\phi * f\right\|_{\infty} \\
& \leq \beta_{\Upsilon}^{\infty}(a)\|\phi * f\|_{\infty} \\
& \leq \beta_{\Upsilon}^{\infty}(a)\left\|_{f}\right\|_{L} .
\end{aligned}
$$

Thus

$$
\begin{aligned}
\left\|_{a} f-f\right\|_{L} & =\sup _{\phi \in Q}\|\phi *(\alpha f-f)\|_{\infty} \\
& \leq \beta_{\uparrow}^{\infty}(a)\|f\|_{L},
\end{aligned}
$$

which gives the required result. //

We shall now examine estimates for $\beta_{\mathrm{T}}^{\infty}(\alpha)$ in two special cases. Our 
first estimate is of the form

$$
\beta_{T}^{\infty}(\alpha)=O\left(\omega_{T}(\alpha)\right\}
$$

(cf. Theorem 1.1.4).

1.4.2 THEOREM. If $T \subset \Gamma$ supports no true pseudomeasure (that is, every pseudomeasure on $\Gamma$ which is supported by $T$ is actually a measure) then

$$
\beta_{T}^{\infty}(a) \leq \kappa \omega_{T}(\alpha)
$$

where $k=k(T)$.

Proof. Let $f \in L_{\Upsilon}^{\infty}(G)$. It follows from Theorem A.2.I and the assumption that $T$ supports no true pseudomeasure that there exists $\mu \in M_{b}(\Gamma)$, supported by $T$, such that

$$
\hat{f}=\mu \text {. }
$$

Consider $g \in C(G)$ defined by

$$
g(x)=\int_{\Gamma} \gamma(x) d \mu(\gamma)
$$

We shall prove that $g=f$ locally almost everywhere.

By Hewitt and Ross [1], (14.12) there exists a $\mu$-measurable function $u$ such that

$$
u d|\mu|=d \mu
$$

and

$$
|u(\gamma)|=1
$$

Let $h \in D(\Gamma)$. Then

$$
\begin{aligned}
\hat{g}(h) & =g\left(\hat{h}_{v}\right) \\
& =\int_{G} g(x) \hat{h}_{v}(x) d \lambda(x) \\
& =\int_{G}\left\{\int_{\Gamma} \gamma(x) u(\gamma) d|\mu|(\gamma)\right\} \hat{h}_{v}(x) d \lambda(x) .
\end{aligned}
$$

Now $\lambda,|\mu|$ are positive measures, the function $\psi$ on $G \times \Gamma$ defined by

$$
\psi:(x, \gamma) \rightarrow \gamma(x) u(\gamma) \hat{h}_{v}(x)
$$


is $\lambda \times|\mu|$-measurable (note that $(x, \gamma) \rightarrow \gamma(x)$ is continuous) and $\psi$ vanishes off a $\lambda \times|\mu|-\sigma$-finite set. Furthermore

$$
\begin{aligned}
\int_{\Gamma}\left\{\int_{G}\left|\gamma(x) u(\gamma) \hat{h}_{v}(x)\right| d \lambda(x)\right\} d|\mu|(\gamma) & =\left\|\hat{h}_{v}\right\|_{I}\|\mu\|_{M} \\
& <\infty .
\end{aligned}
$$

Hence, by Hewitt and Ross [1], (13.10),

$$
\begin{aligned}
\hat{g}(h) & =\int_{\Gamma}\left\{\int_{G} \gamma(x) \hat{h}_{v}(x) d \lambda(x)\right\} d \mu(\gamma) \\
& =\int_{\Gamma} h d \mu(\gamma) \\
& =\hat{f}(h) .
\end{aligned}
$$

As $h \in D(\Gamma)$ was chosen arbitrarily and the Fourier transform is one-toone, $g=f$ locally almost everywhere.

Since $\mu$ is supported by $T$ we see that

$$
\begin{aligned}
|\alpha(x)-f(x)| & =\left|\int_{\Upsilon}(\gamma(-a)-1) \gamma(x) d \mu(\gamma)\right| \quad x-1 . \text { a.e. } \\
& \leq \omega_{\Upsilon}(\alpha) i \mu \|_{M} .
\end{aligned}
$$

But $T$ supports no true pseudomeagare, whence it follows (see R.E. Edwards [3], (3.2)) that $T$ is a Helson set and so (see Hewitt and Ross [1], (41.12)) there exists $k>0$ such that

$$
\|\mu\|_{M} \leq \kappa\|f\|_{\infty}
$$

As $k=k(T)$ is independent of the choice of $f \in L_{T}^{\infty}(G)$ the result follows. //

In the second case we assume that $G$ is totally disconnected; the estimate for $\beta_{\Upsilon}^{\infty}(\alpha)$ now takes a very simple form.

1.4.3 THEOREM. Let $G$ be totally disconnected and suppose $T$ is a relatively compact subset of $\Gamma$. Then there exists a compact open subgroup $H$ of $G$ such that

$$
\beta_{\Upsilon}^{\infty}(\alpha)=0
$$

for azz $a \in H$. 
Proof. Let

$$
V=\{x \in G:|\gamma(x)-1|<\sqrt{3} \text { for all } \gamma \in \Upsilon\} \text {. }
$$

Then $V$ is a neighbourhood of zero in $G$ and hence, since $G$ is assumed to be totally disconnected, there exists a compact open subgroup $H \subset V$. It is clear that

$$
T \subset A(\Gamma, H)
$$

indeed, if $\gamma \in T$ then for all $x \in V$

$$
|\gamma(x)-1|<\sqrt{3}
$$

that is, for all $x \in H$ and $n \in Z$

$$
\left|\gamma(x)^{n}-1\right|<\sqrt{3}
$$

this can only hold if $\gamma(x)=1$.

Now $\nabla=A(\Gamma, H)$ is a compact open subgroup of $\Gamma$ and so we can appeal to Corollary 1.1.3 to deduce that

$$
\begin{aligned}
\beta_{T}^{\infty}(\alpha) & \leq 3\left(\frac{\theta(3 \nabla)}{\theta(\nabla)}\right)^{\frac{1}{2}} \omega_{3 \nabla}(\alpha) \\
& =0
\end{aligned}
$$

for all $a \in H$, thus proving the theorem. /1

\subsection{The case where $\Gamma$ contains a compactly generated open subgroup}

In the preceding sections we have been concerned with obtaining estimates for $\beta_{T}^{L}(\alpha)$ in terms of $\omega_{T},(\alpha)$ for suitable relatively compact sets $T^{\prime} \subset \Gamma$. We can simplify the quantity $\omega_{T},(a)$ whenever $\Gamma$ contains a compactly generated open subgroup.

1.5.1 THEOREM. Let $T, \Omega \subset \Gamma$, where $T$ is compact and $\Omega$ is a compactly generated open subgroup. Then there exists a compact set $\Upsilon_{0} \subset \Gamma$ and a finite set $\Delta \subset T \backslash \Omega$ such that

$$
\omega_{\mathrm{T}}(\alpha) \leq \kappa \omega_{\Upsilon_{0}}(\alpha)+\omega_{\Delta}(\alpha)
$$

where $k=k\left(T, T_{0}, \Omega\right)$. 
Proof. We can assume without loss of generality that $0 \in T$. Since $\{\gamma+\Omega: \gamma \in T\}$ is an open cover of the compact set $T$ there exist $\gamma_{1}, \gamma_{2}, \ldots, \gamma_{n} \in T$. such that

$$
T \subset \bigcup_{s=1}^{n}\left(r_{s}+\Omega\right)
$$

where, without loss of generality, we can assume that $\gamma_{I}=0$ and $\gamma_{s} \notin \Omega$ for $s>1$.

Now

$$
T_{s}=T \cap\left(\gamma_{s}+\Omega\right)
$$

is compact (recall that $\Omega$ is open, hence closed). Furthermore, as $\Omega$ is assumed to be compactly generated, there is (see Hewitt and Ross [1], (5.13)) a relatively compact open neighbourhood $\nabla$ of $0 \in \Gamma$ such that

$$
\Omega=\bigcup_{m=1}^{\infty} m \nabla
$$

Since, for each $s \in\{1,2, \ldots, n\}$,

$$
\Upsilon_{s} \subset \gamma_{s}+\Omega
$$

and $-\gamma_{s}+T_{s}$ is compact, it follows that there is an integer $m_{s}$ such that

$$
\begin{aligned}
-\gamma_{s}+T_{s} & \subset \bigcup_{m=1}^{m_{s}} m \nabla \\
& =m_{s} \nabla .
\end{aligned}
$$

Hence

$$
\omega_{\Upsilon_{s}}(a) \leq\left|\gamma_{s}(a)-1\right|+m_{s} \omega_{\nabla}(a)
$$

and, since $T=\bigcup_{s=1}^{n} T_{s}$ and $\gamma_{I}=0$, it follows that

$$
\begin{aligned}
\omega_{T}(\alpha) & \leq \max _{2 \leq s \leq n}\left|\gamma_{s}(\alpha)-1\right|+\max _{1 \leq s \leq n} m_{s} \omega_{\nabla}(a) \\
& =\omega_{\nabla}(\alpha)+k \omega_{\Upsilon_{0}}(\alpha),
\end{aligned}
$$


where $\Delta=\left\{\gamma_{2}, \gamma_{3}, \ldots, \gamma_{n}\right\}, k=\max _{1 \leq s \leq n} m_{s}$ and $\tau_{0}=\nabla^{-}$.

1.5.2 COROLLARY. If $\Gamma$ is compactly generated and $T \subset \Gamma$ is compact then there exists a compact set $T_{0} \subset \Gamma$ and $k=k\left(T, T_{0}\right)$ such that

$$
\omega_{\mathrm{T}}(\alpha) \leq k \omega_{\mathrm{T}_{0}}(\alpha)
$$

\subsection{An estimate for $\beta_{\mathrm{T}}^{p}(a)$ on compact groups}

In this section we assume that the underlying group $G$ is compact; it need not be Abelian. The notation followed will be that of R.E. Edwards [4].

Given a finite dimensional continuous irreducible unitary representation $U \in \hat{G}$, with representation space $H_{U}, d(U)$ will denote its dimension and $I_{U}$ the identity endomorphism of $H_{U}$. The trace function on $H_{U}$ will be denoted by $\mathrm{Tr}$.

1.6.1 THEOREM. Let $G$ be compact. If $f \in L^{p}(G)(p \in[1, \infty])$ and $\operatorname{supp}(\hat{f})=\left\{U_{1}, U_{2}, \ldots, U_{n}\right\}$ (is finite) then

$$
\left\|_{a} f-f\right\|_{p} \leq n \max _{1 \leq s \leq n} \operatorname{Tr}\left[\left|U_{s}(a)-I_{U_{s}}\right|\right]\|f\|_{p} \quad(a \in G) .
$$

Proof. Since $\operatorname{supp}(\hat{f})=\left\{U_{1}, U_{2}, \ldots, U_{n}\right\}$ we must have

$$
f(x)=\sum_{s=1}^{n} \alpha_{s} \operatorname{Tr}\left[A_{s} U_{s}(x) *\right]
$$

where $\alpha_{s} \in C, A_{s} \in \operatorname{End}\left(H_{U_{s}}\right)$ and $U_{s}(x)^{*}$ denotes the adjoint of $U_{s}(x)$. Let $k \in C(G)$ be defined by

$$
k(x)=\sum_{s=1}^{n} \operatorname{Tr}\left[U_{s}(x) *\right]
$$

Then

$$
\begin{aligned}
& \hat{k}\left(U_{s}\right)=I_{U_{s}}, \quad s \in\{1,2, \ldots, n\} ; \\
& \hat{k}(U)=0, U \in \hat{G}, \quad U \neq U_{s} \text { for each } s \in\{1,2, \ldots, n\} .
\end{aligned}
$$


Hence

$$
f=k * f
$$

and

$$
a^{f}-f=\left(a_{a}-k\right) * f
$$

Now, using the fact that each $U_{S}(x)$ * is unitary (see R.E. Edwards [4], $(A .2 .11))$

$$
\begin{aligned}
\mid a^{k(x)-k(x) \mid} & =\left|\sum_{s=1}^{n} \operatorname{Tr}\left[U_{s}\left(a^{-1} x\right) *-U_{s}(x) *\right]\right| \\
& \leq \sum_{s=1}^{n} \operatorname{Tr}\left[\left|U_{s}\left(a^{-1}\right) *-I_{U_{s}}\right|\right]
\end{aligned}
$$

and so, by $(1.6 .1)$,

$$
\begin{aligned}
& \|f-f\|_{p} \leq\left\|{ }_{a}^{k}-k\right\|_{1}\|f\|_{p} \\
& \leq n \max _{1 \leq s \leq n} \operatorname{Tr}\left[\left|U_{s}\left(a^{-1}\right) *-I_{U_{s}}\right|\right]\|f\|_{p} \\
& =n \max _{1 \leq s \leq n} \operatorname{Tr}\left[\left|U_{s}(a)-I_{U_{s}}\right|\right]\|f\|_{p}
\end{aligned}
$$

Note that we may replace $a^{f}$ by $f_{\alpha}$ in Theorem 1.6.1.

\subsection{An application}

We shall consider estimates for $\beta_{T}^{\infty}(\alpha)$ when $G$ is taken to be the circle group (note that similar results could be formulated for the real line). For the sake of presentation the quantities $\beta_{\mathrm{T}}^{\infty}(\exp (i a))$, $\omega_{T}(\exp (i a))$ and $\exp (i a)^{f}$ will be abbreviated by $\beta_{T}^{\infty}(\alpha), \omega_{T}^{\infty}(\alpha)$ and $a^{f}$ respectively, where $a \in[0,2 \pi)$.

1.7.1 THEOREM. Let $T=\{-n,-n+1, \ldots, n-1, n\}$, where $n \in Z^{+}$. Then, for all $a \in[0,2 \pi)$,

$$
\beta_{\Upsilon}(\alpha) \leq 3 \sqrt{2} \omega_{\Upsilon}(\alpha)
$$


Proof. Since $T$ is an S-set Theorem 1.1 .2 and Remark 1.1 .6 combine to yield

$$
\beta_{\Upsilon}^{\infty}(\alpha) \leq\left\{\frac{\theta(\Upsilon-\nabla)}{\theta(\nabla)}\right)^{\frac{1}{2}}\left(\omega_{\Upsilon-\nabla}(\alpha)+\omega_{\nabla}(\alpha)\right),
$$

where $\nabla$ is any finite subset of $Z$. Taking $\nabla=T$ the result follows. $\quad / /$

It is easily shown that if we use $(1.7 .2)$ then there is no possibility of replacing $3 \sqrt{2}$ by 1 in $(1.7 .1)$. In fact we have:

1.7.2 LEMMA. Let $\uparrow=\{-n,-n+1, \ldots, n-1, n\}$, where $n \in Z^{+}$. Then for any finite set $\nabla \subset Z$ there exists $\delta>0$ such that

$$
\left(\frac{\theta(\Upsilon-\nabla)}{\theta(\nabla)}\right)^{\frac{1}{2}}\left(\omega_{\Upsilon-\nabla}(\alpha)+\omega_{\nabla}(\alpha)\right) \geq \sqrt{2} \omega_{\Gamma}(\alpha) \quad(\alpha \in[0, \delta)) .
$$

Proof. Choose any set $\nabla \subset Z$ with $m$ elements and element of greatest modulus having modulus $p$ say. Clearly $\theta(\nabla)=m$, $\theta(T-\nabla) \geq 2 n+m$ and $m \leq 2 p+1$. Also, since $\sin (x)$ increases with $x$ on $[0, \pi / 2]$, we have that for all $a \in\left[0, \frac{\pi}{p+n}\right]$

$$
\omega_{T-\nabla}(a) \geq \omega_{\Upsilon}(a) \text {. }
$$

There are two cases to consider; firstly, for $m<2 n+1$,

$$
\begin{aligned}
\left(\frac{\theta(\Upsilon-\nabla)}{\theta(\nabla)}\right)^{\frac{1}{2}}\left(\omega_{\Upsilon-\nabla}(\alpha)+\omega_{\nabla}(\alpha)\right) & \geq\left(\frac{2 n+m}{m}\right)^{\frac{1}{2}} \omega_{\Upsilon}(\alpha) \\
& \geq \sqrt{2} \omega_{\Upsilon}(\alpha) \quad\left(\alpha \in\left[0, \frac{\pi}{p+n}\right)\right) .
\end{aligned}
$$

Otherwise, for $m \geq 2 n+1$, we make use of the inequality

$$
|\sin (\alpha)| \geq \frac{2}{\pi}|\alpha|
$$

to obtain

$$
\begin{aligned}
\omega_{\nabla}(\alpha) & =\max _{s \in \nabla}\left|\sin \left(\frac{s a}{2}\right)\right| \\
& \geq \frac{2}{\pi} \quad \max _{s \in \nabla}\left|\frac{s a}{2}\right| \\
& =\frac{p}{\pi}|a|
\end{aligned}
$$$$
\left\{\left|\frac{p a}{2}\right| \leq \pi / 2\right\}
$$ 
and similarly

$$
\omega_{T-\nabla}(a) \geq \frac{p+n}{\pi}|\alpha| \quad \quad\left\{\left|\frac{(p+n) a}{2}\right| \leq \pi / 2\right\} .
$$

Hence, for $a \in\left[0, \frac{\pi}{p+n}\right]$,

$$
\begin{aligned}
\left(\frac{\theta(T-\nabla)}{\theta(\nabla)}\right)^{\frac{1}{2}}\left(\omega_{T-\nabla}(\alpha)+\omega_{\nabla}(\alpha)\right) & \geq \frac{1}{\pi}\left(1+\frac{2 n}{m}\right)^{\frac{1}{2}}(2 p+n)|\alpha| \\
& \geq \frac{2}{\pi}\left(1+\frac{2 n}{m}\right)^{\frac{1}{2}} \frac{m+n-1}{n}\left|\frac{n a}{2}\right| \\
& \geq \frac{2}{\pi}\left\{1+\frac{2 n}{m}\right)^{\frac{1}{2}} \frac{m+n-1}{n} \sin \left(\left|\frac{n \alpha}{2}\right|\right) \\
& =\frac{2}{\pi}\left(1+\frac{2 n}{m}\right)^{\frac{1}{2}} \frac{m+n-1}{n} \omega_{\Upsilon}(\alpha) \\
& \geq \frac{6}{\pi} \omega_{T}(\alpha) \\
& \geq \sqrt{2} \omega_{\Upsilon}(a),
\end{aligned}
$$

and the result follows with $\delta=\frac{\pi}{p+n} \cdot \quad / /$

It might be conjectured that some more refined argument would yield the constant 1 in (1.7.1). However we shall demonstrate that this is, in general, not possible by exhibiting finite sets $\uparrow$ (each containing zero) such that

$$
\beta_{T}^{\infty}(\alpha) \leq \alpha w_{T}(\alpha)
$$

implies that $\alpha>1$. We require some preliminary results.

1.7.3 THEOREM. Let $m, n \in Z$, where $|m|>|n|>0$. Then there exist non-zero $\alpha, \beta \in Z$ such that

$$
\left\|1+\alpha e_{n}+\beta e_{m}\right\|_{\infty}<|\alpha|+|\beta|,
$$

where $e_{n}: x \rightarrow \exp (i n x)$.

Proof. It will be sufficient to consider the case $m>n>0$. We put $\alpha=m s, \beta=-n s$, and show that $s \in Z$ can be chosen so that (1.7.3) holds. 


$$
f=1+m s e_{n}-n s e_{m} \text {, }
$$

where $s \in Z \backslash\{0\}$, and define $h$ on $R$ by

$$
\begin{aligned}
h(x) & =|f(x)|^{2} \\
& =1+m^{2} s^{2}+n^{2} s^{2}+2 m s \cos (n x)-2 n s \cos (m x)-2 m n s^{2} \cos ((m-n) x) .
\end{aligned}
$$

We see that $h$ is continuous and periodic with period at most $2 \pi$. Hence $h$ must attain its maximum on $[0,2 \pi)$ and thus, in order to verify (1.7.3), all we have to show is that if $h\left(x_{0}\right)=\|h\|_{\infty}$ then, for a suitable choice of $s$,

$$
h\left(x_{0}\right)<s^{2}(m+n)^{2}
$$

(recall that $h \geq 0$ ). It appears that there are two cases to consider:

$$
\begin{aligned}
& \cos \left((m-n) x_{0}\right) \neq-1 ; \\
& \cos \left((m-n) x_{0}\right)=-1 .
\end{aligned}
$$

If $(1.7 .5)$ holds then

$$
\begin{aligned}
h\left(x_{0}\right) & =s^{2}\left(m^{2}+n^{2}-2 m n \cos \left((m-n) x_{0}\right)\right)+s\left(2 m \cos \left(n x_{0}\right)-2 n \cos \left(m x_{0}\right)\right)+1 \\
& <s^{2}(m+n)^{2}
\end{aligned}
$$

provided $|s|$ is taken sufficiently large, say $|s| \geq s_{1}$.

Otherwise, if $(1.7 .6)$ holds, consider

$$
\begin{aligned}
0 & =h^{\prime}\left(x_{0}\right) \\
& =-2 m n s \sin \left(n x_{0}\right)+2 m n s \sin \left(m x_{0}\right)+2 m n(m-n) s^{2} \sin \left((m-n) x_{0}\right) \\
& =2 m n s\left(\sin \left(m x_{0}\right)-\sin \left(n x_{0}\right)+s(m-n) \sin \left((m-n) x_{0}\right)\right) \\
& =4 m n s \sin \left(\frac{m-n}{2} x_{0}\right)\left(\cos \left(\frac{m+n}{2} x_{0}\right)+s(m-n) \cos \left(\frac{m-n}{2} x_{0}\right)\right) .
\end{aligned}
$$

Thus either

$$
\sin \left(\frac{m-n}{2} x_{0}\right)=0
$$

or 


$$
\cos \left(\frac{m+n}{2} x_{0}\right)+s(m-n) \cos \left(\frac{m-n}{2} x_{0}\right)=0 .
$$

Now it is impossible for both $(1.7 .6)$ and (1.7.7) to be true; consequently $(1.7 .8)$ holds and since, by $(1.7 .6)$,

$$
(m-n) x_{0}=(2 t+1) \pi
$$

for some $t \in Z$, we have that

$$
\cos \left(\frac{m+n}{2} x_{0}\right)=0
$$

that is,

$$
(m+n) x_{0}=\left(2 t^{\prime}+1\right) \pi
$$

for some $t^{\prime} \in Z$. It follows that for any $s \in Z$ such that

$$
\operatorname{sign}(s)=(-1)^{t+t^{\prime}+1} \text {, }
$$

we have

$$
\begin{aligned}
h\left(x_{0}\right) & =s^{2}(m+n)^{2}+2 s\left(m(-1)^{t^{\prime}-t}+n(-1)^{t+t^{\prime}}\right)+1 \\
& =s^{2}(m+n)^{2}-2|s|(m+n)+1 \\
& <s^{2}(m+n)^{2} .
\end{aligned}
$$

Now put

$$
s=\left|s_{1}\right| \operatorname{sign}\left((-1)^{t+t^{\prime}+1}\right)
$$

Then (1.7.3) is satisfied with $\alpha=m s$ and $\beta=-n s . \quad / /$

\subsubsection{COROLLARY. Let}

$$
T=\left\{0, n_{1}, \ldots, n_{s}, m\right\},
$$

where $0<\left|n_{1}\right|<\ldots<\left|n_{s}\right|<m$. Suppose $s$ is a transtation-invariant subspace of $L^{\infty}(T)$ such that for all $f \in S$ and $a \in(\pi / m, 2 \pi / m)$

$$
\|\|_{a} f-f\left\|_{\infty} \leq \omega_{T}(a)\right\| f \|_{\infty}
$$

Then $1, e_{m} \in S$ implies that $e_{n_{s}} \notin S$.

Proof. Suppose to the contrary that $1, e_{n_{s}}, e_{m} \in S$. By Theorem 
1.7.3 we can find $\alpha, \beta \in Z$ such that

$$
\left\|1+\alpha e_{n_{s}}+\beta e_{m}\right\|_{\infty}<|\alpha|+|\beta| \text {. }
$$

Furthermore

$$
f=1+\alpha e_{n_{s}}+\beta e_{m} \in S \text {. }
$$

Hence, for all $a \in(\pi / m, 2 \pi / m)$,

$$
\left\|_{\alpha} f-f\right\|_{\infty} \leq \omega_{\mathrm{T}}(\alpha)\|f\|_{\infty},
$$

that is, using Kronecker's theorem (see Katznelson [1], Chapter VI, (9.2)),

$$
|\alpha|\left|\exp \left(-i n_{s} \alpha\right)-1\right|+|\beta||\exp (-i m a)-1|<\omega_{\Upsilon}(\alpha)(|\alpha|+|\beta|)
$$

for all $a \in(\pi / m, 2 \pi / m)$ (in which case note that $\omega_{T}(\alpha) \neq 0$ ).

$$
\begin{aligned}
& \text { Now, since } 0<\left|n_{s}\right|<m, \\
& \qquad\left|\exp \left(-i m \frac{2 \pi}{m}\right)-1\right|=0,\left|\exp \left(-i n_{S} \frac{2 \pi}{m}\right)-1\right|>0 \\
& \left|\exp \left(-i m \frac{\pi}{m}\right)-1\right|=2,\left|\exp \left(-i n_{s} \frac{\pi}{m}\right)-1\right|<2
\end{aligned}
$$

and so we infer (using the Intermediate Value theorem) the existence of $a_{0} \in(\pi / m, 2 \pi / m)$ such that

$$
\left|\exp \left(-i m a_{0}\right)-1\right|=\left|\exp \left(-i n_{s} a_{0}\right)-1\right|
$$

or, equivalently,

$$
\left|\sin \left(\frac{m a}{2}\right)\right|=\left|\sin \left(\frac{n_{s} a_{0}}{2}\right)\right| \text {. }
$$

As $\frac{m a_{0}}{2} \in(\pi / 2, \pi)$ and $\frac{\left|n_{s}\right| a_{0}}{2} \in(0, \pi)$ we must have

$$
\frac{\left|n_{s}\right| \alpha_{0}}{2}=\pi-\frac{m a_{0}}{2} \in(0, \pi / 2),
$$

and it follows that

$$
\omega_{T}\left(a_{0}\right)=\left|\exp \left(-i m a_{0}\right)-1\right|
$$

Thus (1.7.9) and (1.7.10) imply that

$$
|\alpha|+|\beta|<|\alpha|+|\beta|
$$


(recall that $\omega_{\Gamma}\left(a_{0}\right) \neq 0$ ) and we have a contradiction. //

We can now characterise those finite sets $T \subset Z$ containing zero for which the constant $3 \sqrt{2}$ in $(1.7 .1)$ can be replaced by 1 .

1.7.5 THEOREM. Srppose $T \subset Z$ is finite and $0 \in T$. Then

$$
\beta_{\Upsilon}^{\infty}(\alpha) \leq \omega_{\Upsilon}(\alpha)
$$

for azz $a \in[0,2 \pi)$ if and only if

$$
\Upsilon \subset\{0,-n, n\}
$$

for some $n \in Z^{+}$.

Proof. Suppose that $T \subset\{0,-n, n\}$, where $n \in Z^{+}$. We can assume that $T=\{0,-n, n\}$. Put

$$
f=1+\alpha e_{-n}+\beta e_{n},
$$

where $\alpha, \beta \in C$. Then

$$
f^{\prime}=-\alpha i n e_{-n}+\text { Bine }_{n}
$$

and, using Kronecker's theorem,

$$
\left\|f^{\prime}\right\|_{\infty}=n(|\alpha|+|\beta|) .
$$

But Bernstein's inequality gives

$$
\left\|f^{\prime}\right\|_{\infty} \leq n\|f\|_{\infty},
$$

whence ( ince $n \neq 0$ ) it follows (once more using Kronecker's theorem) that

$$
\begin{aligned}
\left\|_{a^{f}}-f\right\|_{\infty} & =|\alpha||\exp (i n a)-1|+|\beta||\exp (-i n \alpha)-1| \\
& \leq \omega_{\Upsilon}(a)\|f\|_{\infty} .
\end{aligned}
$$

Conversely, if $T \notin\{0,-n, n\}$ for any $n \in Z^{+}$, then (taking $-T$ in place of $T$ if necessary) we have

$$
T^{\prime}=\left\{0, n_{1}, \ldots, n_{s}, m\right\} \subset T \subset\left\{\theta_{2} n_{1}, \ldots, n_{s}, m_{s}-m\right\}
$$

for some $m, n_{1}, \ldots, n_{s} \in Z$, where $m>\left|n_{s}\right|>\ldots>\left|n_{1}\right|>0$. Now $L_{T}^{\infty},(T)$ is a translation-invariant subspace of $L^{\infty}(T)$ satisfying (1.7.11). Furthermore $I, e_{n_{s}}, e_{m} \in L_{T}^{\infty}(T)$, contradicting corollary 1.7.4. 


\section{CHAPTER 2}

\section{A CONVERSE OF BERNSTEIN'S INEQUALITY FOR LOCALLY COMPACT GROUPS}

\subsection{Introduction}

In Chapter 1 we have been investigating inequalities of the form

$$
\beta_{T}^{L}(\alpha) \leq k \omega_{T},(a)
$$

where $a \in G, T, T^{\prime} \subset \Gamma$ are relatively compact, $L$ is a translationinvariant subspace of some $L^{p}(G)$, which satisfies both (1.1.1) and (1.1.2), and $k \geq 0$ depends on $T$ and $T^{\prime}$. The space of functions for which inequalities of the type $(2.0 .1)$ have been found is $L_{T}(G)$, and all of the proofs given depend to some extent on the fact that

$$
U\left\{\Sigma(f): f \in L_{T}(G)\right\}
$$

is relatively compact.

It is the aim of this chapter to show that the above dependence is necessary to obtain inequalities of the form (2.0.1); more precisely, we shall prove that if $S$ is a translation-invariant subspace of $L^{p}(G)$ $(p \in[1, \infty])$ and

$$
\omega(a)=\sup \left\{\|\|_{a} f-f\left\|_{p}: f \in S,\right\| f \|_{p} \leq 1\right\}
$$

is such that $\lim \omega(a)=0$, then $U\{\Sigma(f): f \in S\}$ is relatively compact. $a \rightarrow 0$

\subsection{The case where $G$ is Abelian}

We shall require two technical lemmas before embarking upon the proof of the result stated immediately above. The first lemma is a very special case of the Wiener-Lévy theorem.

2.1.1 LEMMA. Suppose we are given $\gamma \in \Gamma$ and $k \in L^{1}(G)$ such that 
$\hat{k}(\gamma)=1$. Then for any $\varepsilon>0$ we can find $l \in L^{I}(G)$ such that $\hat{k} \hat{l}=1$ on a neighbourhood of $\gamma$ and. $\|z\|_{1}<1+\varepsilon$.

Proof. Choose $\delta \in(0,1)$ satisfying

$$
\delta(1-\delta)^{-1}<\varepsilon / 2 \text {. }
$$

Since $(\bar{\gamma} k)^{\wedge}(0)=1$, Reiter [1], Chapter 5, 2.3 (5) asserts the existence of $h \in L^{l}(G)$ such that $\|h\|_{1}<1+\varepsilon / 2, \hat{h}=1$ on a neighbourhood of zero, and

$$
\|(\bar{\gamma} k) * h-h\|_{1}<\delta .
$$

Putting $h_{\gamma}=\gamma h,(2.1 .2)$ yields

$$
\left\|k * h_{\gamma}-h_{\gamma}\right\|_{1}<\delta
$$

and clearly $\hat{h}_{\gamma}=1$ on a neighbourhood $\Omega_{\gamma}$ of $\gamma$ and $\left\|h_{\gamma}\right\|_{1}<1+\varepsilon / 2$. As $\delta<1$ it appears from (2.1.3) that the series

$$
h_{\gamma}+\sum_{n \geq 1}(-1)^{n}\left(k * h_{\gamma}-h_{\gamma}\right)^{* n}
$$

converges in $L^{I}(G)$ to $\mathcal{l}$ say. For $x \in \Omega_{\gamma}$ we have

$$
\begin{aligned}
\hat{k}(x) \hat{\imath}(x) & =\hat{k}(x)\left(1+\sum_{n \geq 1}(-1)^{n}(\hat{k}(x)-1)^{n}\right) \\
& =1 .
\end{aligned}
$$

A combination of $(2.1 .1),(2.1 .3)$ and $(2.1 .4)$ gives us

$$
\begin{aligned}
\|Z\|_{1} & \leq\left\|h_{\gamma}\right\|_{I}+\sum_{n \geq 1} \delta^{n} \\
& <1+\varepsilon / 2+\delta(1-\delta)^{-1} \\
& <1+\varepsilon . \quad / /
\end{aligned}
$$

2.1.2 LEMMA. Let $\delta \in(0,1)$. Suppose that $\gamma \in \Gamma$ and $a \in G$ satisfy

$$
|\gamma(\alpha)-1|>1-\delta \text {. }
$$


Then we can find $\phi, \psi$ in $L^{1}(G)$ such that $\hat{\phi}=1$ on a neighbourhood of $\gamma$,

$$
\phi=a^{\psi-\psi}
$$

and

$$
\|\psi\|_{1}<(1-\delta)^{-1}(1+\delta)
$$

Proof. By Theorem B.I.I we can find $k \in L^{l}(G)$ such that $\hat{k}(\gamma)=1$ and $\|k\|_{1}=1$. Since

$$
(\bar{\gamma}(a)-1)^{-1}\left({ }_{a} k-k\right) \wedge(\gamma)=1
$$

we can appeal to Lemma 2.1.1 to deduce the existence of $l \in L^{l}(G)$ such that $\|Z\|_{I}<I+\delta$ and

$$
(\bar{\gamma}(a)-1)^{-1}\left(a_{a}-k\right)^{\wedge} \hat{\imath}=1
$$

on a neighbourhood of $\gamma$. Now put

$$
\psi=(\bar{\gamma}(\alpha)-1)^{-I_{k} * z}
$$

and

$$
\phi=a^{\psi-\psi}
$$

Then (2.1.5) shows that $\hat{\phi}=1$ on a neighbourhood of $\gamma$, and clearly

$$
\begin{aligned}
\|\psi\|_{1} & \leq|\gamma(\alpha)-I|^{-1}\|k\|_{1}\|z\|_{1} \\
& <(1-\delta)^{-1}(1+\delta) .
\end{aligned}
$$

We can now prove:

2.1.3 THEOREM. Suppose that $S$ is a translation-invariant subspace of $L^{p}(G) \quad(p \in[1, \infty])$, that for alz $a \in G$

$$
\omega(a)=\sup \left\{\|\|_{a} f-f\left\|_{p}: f \in S,\right\| f \|_{p} \leq 1\right\} \text {, }
$$

and that $\lim _{a \rightarrow 0} \omega(\alpha)=0$. Then $\Delta=U\{\Sigma(f): f \in S\}$ is relatively compact. 
can assume that $S$ is closed.

Suppose that $\Delta$ is not relatively compact. Then, if $V$ is any neighbourhood of zero in $G$ and $\delta>0$ is given, we can find $a_{V} \in V$, $f_{V} \in S$ and $\gamma_{V} \in \Sigma\left(f_{V}\right\}$ such that

$$
\left|\gamma_{V}\left(a_{V}\right)-1\right|>1-\delta
$$

(for if $|\gamma(a)-l| \leq 1-\delta$ for all $a \in V$ and all $\gamma \in \Delta$, we could appeal to (23.16) of Hewitt and Ross [1] to deduce that $\Delta^{-}$is compact, contrary to assumption). Taking $\delta=1 / 4$, and recalling (2.1.7), Lemma 2.1.2 implies the existence of an open neighbourhood $\Omega_{V}$ of $\gamma_{V}$, and $\phi_{V}$, $\psi_{V}$ in $L^{1}(G)$ such that $\hat{\phi}_{V}=1$ on $\Omega_{V}$,

$$
\phi_{V}=a_{V} \psi_{V}-\psi_{V}
$$

and $\left\|\psi_{V}\right\|_{I}<2$

$$
\text { Choose any } k_{V} \in L_{\Omega_{V}}^{I}(G) \text { such that } \hat{k}_{V}\left(\gamma_{V}\right)=1 \text {. Using the definitions of }
$$

of $\phi_{V}$ and $\psi_{V}$ we have

$$
\begin{aligned}
k_{V} * f_{V} & =\phi_{V} * k_{V} * f_{V} \\
& =\left(a_{V} \psi_{V}-\psi_{V}\right) * k_{V} * f_{V} \\
& =\psi_{V} *\left(a_{V} k_{V}-k_{V}\right) * f_{V} .
\end{aligned}
$$

Since $S$ is assumed to be a closed translation-invariant subspace of $L^{p}(G)$ the proof of Reiter [1], Chapter 3, 5.8 can be used to show that

$$
h * f_{V} \in S
$$

for all $h \in L^{1}(G)$ (note that for $p=\infty$ it follows from (2.1.6), the assumption that $\lim _{a \rightarrow 0} w(\alpha)=0$ and the main result of D.A. Edwards [1] that $f_{V}$ is equal locally almost everywhere to a uniformly continuous function). combining $(2.1 .6),(2.1 .8)$ and $(2.1 .9)$, 


$$
\begin{aligned}
\left\|k_{V} * f_{V}\right\|_{p} & \leq\left\|\psi_{V}\right\|_{1}\left\|_{a_{V}} k_{V} * f_{V}-k_{V} * f_{V}\right\|_{p} \\
& \leq 2 \omega\left\{a_{V}\right\}\left\|k_{V} * f_{V}\right\|_{p} .
\end{aligned}
$$

As $\gamma_{V} \in \sum\left\{f_{V}\right)$ and $\hat{k}_{V}\left(\gamma_{V}\right\} \neq 0$ we see that $k_{V} * f_{V} \neq 0$ and so, by $(2.1 .10)$,

$$
\omega\left(a_{V}\right) \geq 1 / 2
$$

Now consider the net $\left(\alpha_{V}\right)$, where $V$ ranges over the set of neighbourhoods of zero, partially ordered by

$$
V \prec V^{\prime} \text { if and only if } V \supset V^{\prime} \text {. }
$$

Clearly we have that $\left(a_{V}\right)$ converges to 0 ; but (2.1.11) holds for all $V$, contradicting the assumption that $\lim _{a \rightarrow 0} \omega(\alpha)=0$. Hence our assumption that $\Delta$ is not relatively compact was false. //

We easily obtain the following analogous result for $M_{b}(G)$.

2.1.4 COROLLARY. Suppose that $S$ is a translation-invariant subspace of $M_{b}(G)$, that

$$
\omega(\alpha)=\sup \left\{\left\|_{\alpha} \mu-\mu\right\|_{M}: \mu \in S,\|\mu\|_{M} \leq 1\right\}
$$

and that $\lim _{\alpha \rightarrow 0} \omega(\alpha)=0$. Then $U\{\operatorname{supp}(\hat{\mu}): \mu \in S\}$ is relatively compact.

$$
\text { Proof. It follows from the assumption that } \underset{\alpha \rightarrow 0}{\lim } \omega(\alpha)=0 \text { and R.E. }
$$

Edwards [1], Corollary 3 that any $\mu \in S$ is generated by an $L^{l}$-function. Let

$$
S^{\prime}=\left\{f \in L^{l}(G): f \text { generates a measure in } S\right\} \text {. }
$$

Then $S^{\prime}$ is a translation-invariant subspace of $L^{l}(G)$ satisfying the conditions of Theorem 2.1.3, from which we deduce that $U\left\{\Sigma(f): f \in S^{\prime}\right\}$ is relatively compact. Since $\hat{f}=\hat{\mu}_{f}$, where $\mu_{f}$ is the measure generated by $f$, and any $\mu \in S$ is $\mu_{f}$ for some $f \in S^{\prime}$, we can conclude (note that 
for $f \in L^{l}(G)$ we have $\Sigma(f)=\operatorname{supp}(\hat{f}) ;$ see Theorem A.2.1) that $U\{\operatorname{supp}(\hat{\mu}): \mu \in S\}$ is relatively compact. //

\subsection{A more refined result for $L^{\top}(G)$ and $C_{u}(G)$}

It appears that for $L^{l}(G)$ and $C_{u}(G)$ there is a result similar to that of Theorem 2.1.3 which only requires that $w$ should satisfy

$$
\omega(\alpha)<\alpha<1
$$

for all $\alpha \in C$, where $C \subset G$ is a compact set with $\lambda(C)>0$ and $\alpha \in(0,1)$. For $L^{l}(G)$ we need the following preliminary result:

2.2.1 LEMMA. Let $S$ be an ideal of $L^{l}(G)$ and suppose $\gamma \in \Gamma$ and $f \in S$ are such that $\hat{f}(\gamma) \neq 0$. Then there exists $k \in S$ such that $\hat{k}(\gamma)=1$ and $\|k\|_{1}=1$.

Proof. We put

$$
\Omega=\left(x \in \Gamma:|\hat{f}(x)|>\frac{1}{2}|\hat{f}(\gamma)|\right) \text {. }
$$

Since $\hat{f}$ is continuous we must have that $\Omega$ is open. Now we can find a relatively compact open set $\Omega^{\prime}$ such that

$$
\gamma \in \Omega^{\prime} \subset \Omega^{\prime^{-}} \subset \Omega,
$$

and an open symmetric neighbourhood $\nabla$ of zero such that

$$
\gamma+2 \nabla \subset \Omega^{\prime}
$$

As $\hat{f}$ is bounded away from zero on $\Omega$ we can use the Wiener-Lévy theorem to deduce the existence of $g \in L^{l}(G)$ such that $\hat{f g}=1$ on $\Omega^{1^{-}}$. Furthermore, by Theorem B.I.I, we can find $k \in L^{l}(G)$ such that $\hat{k}(\gamma)=1$, $\operatorname{supp}(\hat{k}) \subset \gamma+2 \nabla$ and $\|k\|_{I}=1$. Hence

$$
k=k * f * g
$$

and, since $S$ is an ideal, it must contain $k$. // 
2.2.2 THEOREM. Suppose that $S$ is a translation-invariant subspace of $L^{1}(G)$ and that

$$
\omega(a)=\sup \left\{\left\|_{a} f-f\right\|_{1}: f \in S,\|f\|_{1} \leq 1\right\}
$$

satisfies (2.2.1) for some $\alpha \in(0,1)$ and compact set $C \subset G$ with $\lambda(C)>0$. Then $U\{\operatorname{supp}(\hat{f}): f \in S\}$ is relatively compact.

Proof. Since $\omega(\alpha)$ is unchanged if we replace $S$ by $S^{-}$we can assume that $S$ is closed.

Put

$$
\Omega=\{x \in \Gamma: \hat{f}(x) \neq 0 \text { for some } f \in S\}
$$

and choose $\gamma \in \Omega$. Since $S$ is a closed translation-invariant subspace of $L^{I}(G)$ it must be a closed ideal (see Reiter [1], Chapter 3, 5.8) and hence we can appeal to Lemma 2.2.1 to deduce the existence of $k \in S$ such that $\hat{k}(\gamma)=1$ and $\|k\|_{1}=1$. Then

$$
\begin{aligned}
\left|\left(a^{k}-k\right)^{\wedge}(\gamma)\right| & \leq\left\|a_{a}^{k}-k\right\|_{I} \\
& \leq \omega(a)\|k\|_{1},
\end{aligned}
$$

that is, for all $\gamma \in \Omega$ and $a \in G$

$$
|\gamma(\alpha)-1| \leq \omega(a) \text {. }
$$

Now let

$$
\Pi(C, \alpha)=\{\chi \in \Gamma:|\chi(\alpha)-1|<\alpha \text { for all } \alpha \in C\} .
$$

Clearly, using (2.2.2) and the fact that $\omega$ satisfies (2.2.1),

$$
\Omega \subset \Pi(C, \alpha)
$$

and since (see Hewitt and Ross $[1],(23.16)$ ) $\Pi(C, \alpha)$ is relatively compact, so is $\Omega$. //

For $\mathcal{C}_{u}(G)$ the proof takes a different form.

2.2.3 THEOREM. Suppose that $S$ is a transtation-invariant subspace. of $C_{u}(G)$ and that

$$
\omega(\alpha)=\sup \left\{\left\|_{a} f-f\right\|_{\infty}: f \in S,\|f\|_{\infty} \leq 1\right\}
$$


satisfies (2.2.1) for some $\alpha \in(0,1)$ and compact set $C \subset G$ with $\lambda(C)>0$. Then $\Delta=U\{\Sigma(f): f \in S\}$ is relatively compact.

Proof. Choose any $f \in S$ and $\gamma \in \Sigma(f)$. Since $f$ is uniformly

continuous we can use Domar [1], 4.42 to deduce that $\gamma$ is the narrow limit of finite linear combinations of translates of $f$; in particular, given $\varepsilon>0$ and $a \in G$, we can find $\alpha_{n} \in G, \alpha_{n} \in C \quad(n=1,2, \ldots, m)$ such that

$$
\left|\gamma(x)-\sum_{n=1}^{m} \alpha_{n} a_{n} f(x)\right|<\varepsilon \quad(x \in\{0, a\})
$$

and

$$
\left\|\sum_{n=1}^{m} a_{n} a_{n} f\right\|_{\infty}<1+\varepsilon
$$

Then

$$
\begin{aligned}
|\gamma(\alpha)-1| \leq\left|\gamma(\alpha)-\sum_{n=1}^{m} \alpha_{n} a_{n} f(\alpha)\right|+\left|\sum_{n=1}^{m} \alpha_{n} a_{n} f(a)-\sum_{n=1}^{m} \alpha_{n a_{n}} f(0)\right| & +\left|\sum_{n=1}^{m} \alpha_{n a_{n}} f(0)-1\right| \\
& <2 \varepsilon+\left\|\left(\sum_{-a}^{m} \alpha_{n=1}^{m} a_{n} f\right)-\sum_{n=1}^{m} \alpha_{n} a_{n} f\right\|_{\infty}
\end{aligned}
$$

and, as $\sum_{n=1}^{m} \alpha_{n} \alpha_{n} f \in S$, we have

$$
|\gamma(\alpha)-1|<2 \varepsilon+\omega(\alpha)(1+\varepsilon) \text {. }
$$

Since $\varepsilon>0$ was chosen arbitrarily

$$
|\gamma(a)-1| \leq \omega(a) ;
$$

this inequality holds for all $a \in G$ and $\gamma \in \Delta$.

The result now follows using the argument in the last paragraph of the proof of Theorem 2.2.2. 


\subsection{The case where $G$ is compact}

In this section we follow the notation in R.E. Edwards [4] (some of which has already been introduced in 1.6$)$. In addition we let $(E(G),\|\cdot\|)$ denote any of the spaces $L^{P}(G)(p \in[1, \infty))$ or $C(G)$, each taken with its usual norm. The following two results are stated for left translates although, clearly, they hold equally well for right translates.

2.3.1 THEOREM. Suppose that $S$ is a left translation-invariant subspace of $E(G)$, that

$$
\omega(a)=\sup \left\{\left\|_{a} f-f\right\|: f \in S,\|f\| \leq 1\right\}
$$

and that $\lim _{a \rightarrow 0} \omega(a)=0$. Then $U\{\operatorname{supp}(\hat{f}): f \in S\}$ is finite.

Proof. As $\omega$ is unchanged if we replace $S$ by $S^{-}$we can assume that $S$ is closed.

Consider the unit disc in $S$;

$$
Z=\{f \in S:\|f\| \leq I\} \text {. }
$$

It follows immediately from the Weil criterion or, when $E(G)=C(G)$, from Ascoli's theorem that $Z$ is compact in $E(G)$. We can now use the Riesz theorem (R.E. Edwards [2], P. 65) to deduce that $S$ is finite dimensional. Let $\left\{f_{1}, f_{2}, \ldots, f_{n}\right\}$ be a basis for $S$. Since, for every $f \in S$,

$$
\operatorname{supp}(\hat{f}) \subset \bigcup_{m=1}^{n} \operatorname{supp}\left(\hat{f}_{m}\right)
$$

it will suffice to show that $\operatorname{supp}\left(\hat{f}_{m}\right)$ is finite for all $m \in\{1,2, \ldots, n\}$.

However, if this were false, there would exist $m \in\{1,2, \ldots, n\}$ and an infinite sequence $\left\{U_{S}\right\}_{S=1}^{\infty}$ of distinct elements of $\hat{G}$ such that $\hat{f}_{m}\left(U_{s}\right) \neq 0$ for every $s \in\{I, 2, \ldots\}$. Define $h_{s} \in C(G)$ by

$$
h_{S}(x)=d\left(U_{S}\right) \operatorname{Tr}\left[U_{S}(x) *\right]
$$


Since $S$ is assumed to be a closed left translation-invariant subspace of $E(G)$ it is a left ideal (in $E(G)\}$; hence $h_{S} * f_{m} \in S$ for every $s \in\{1,2, \ldots\}$. Also

$$
\begin{aligned}
\left(h_{s} * f_{m}\right)^{\wedge}\left(U_{t}\right) & =\hat{h}_{s}\left(U_{t}\right) \hat{f}_{m}\left(U_{t}\right) \\
& =\delta_{s t} \hat{f}_{m}\left(U_{t}\right),
\end{aligned}
$$

where

$$
\delta_{s t}= \begin{cases}I_{U} & (s=t) \\ 0 & (s \neq t) .\end{cases}
$$

We see that $\left\{h_{s} * f_{m}\right\}_{s=1}^{\infty}$ is linearly independent in $S$; for suppose there exist $\alpha_{s} \in C$ such that

$$
\sum_{s=1}^{s^{\prime}} \alpha_{s}\left(h_{s} * f_{m}\right)=0 .
$$

Then for all $t$

$$
\sum_{s=1}^{s^{\prime}} \alpha_{s}\left(h_{s} * f_{m}\right)^{\wedge}\left(U_{t}\right)=0
$$

and, by (2.3.1),

$$
\sum_{s=1}^{s^{\prime}} \alpha_{s} \delta_{s t} \hat{f}_{m}\left(U_{t}\right)=0,
$$

that is,

$$
\alpha_{t} I_{U_{t}} \hat{f}_{m}\left(U_{t}\right)=0
$$

Since $\hat{f}_{m}\left\{U_{t}\right\} \neq 0$ it follows that $\alpha_{t}=0$ for all $t$. Hence $\left\{h_{s} * f_{m}\right\}_{s=1}^{\infty}$ is linearly independent in $S$, contradicting the fact that $S$ is finite dimensional.

Consequently $\operatorname{supp}\left(\hat{f}_{m}\right\}$ is finite for all $m \in\{1,2, \ldots, n\}$, and the theorem is proved.

2.3.2 COROLLARY. Suppose that $S$ is a left translation-invamiant 
47

subspace of $L^{\infty}(G)$, that for all $a \in G$

$$
\omega(a)=\sup \left\{\left\|_{a} f-f\right\|_{\infty}: f \in S,\|f\|_{\infty} \leq 1\right\},
$$

and that $\lim _{a \rightarrow 0} \omega(a)=0$. Then $U\{\operatorname{supp}(\hat{f}): f \in S\}$ is finite.

Proof. It follows from (2.3.2) and the proof of the main result of $D$.

A. Edwards [I] that every $f \in S$ is equal almost everywhere to a (uniformly)

continuous function. The problem is then reducible to that covered by the case $E(G)=C(G)$ of Theorem 2.3.1. // 
CHAPTER 3

\section{A DERIVATIVE FORM OF BERNSTEIN'S INEQUALITY AND ITS CONVERSE FOR LOCALLY COMPACT ABELIAN GROUPS}

\subsection{Introduction}

The type of estimate obtained for $\beta_{\Upsilon}^{L}(\alpha)$ in Chapter 1 can be linked with the "classical" Bemstein inequality by considering differentiation along a one-parameter subgroup of $G$.

Let $H$ be a one-parameter subgroup of $G$, that is $H=\rho(R)$, where $\rho$ is a continuous homomorphism of $R$ into $G$. We define $f \in L(G)$ (with $L(G)$ as in 1.1 ) to be L-differentiable along $\rho$ if and only if

$$
D_{\rho}^{L} f=\lim _{r \rightarrow 0} r^{-1}(-\rho(r) f-f)
$$

exists in $L(G)$. The subspace of $L(G)$ of all those functions which are L-differentiable along $\rho$ will be denoted by $D_{\rho}^{L}(G)$. We shall also abbreviate $D_{\rho}^{L^{p}} \quad\left\{\right.$ respectively $D_{\rho}^{L^{p}}(G)$ ) to $D_{\rho}^{p^{p}}$ (respectively $D_{\rho}^{p}(G)$ ).

Using (1.1.1) and $(1.1 .2)$ it is easily shown that if $k \in D_{\rho}^{l}(G)$ and $f \in D_{\rho}^{L}(G)$ then $k * f \in D_{\rho}^{L}(G)$ and

$$
D_{\rho}^{L}(k * f)=D_{\rho}^{\perp} k * f=k * D_{\rho}^{L} f
$$

Similarly for $p, q, r \in[1, \infty]$ satisfying $p^{-1}+q^{-1}-r^{-1}=1$ (with the usual convention in case any of $p, q$ or $r$ equals $\infty$ ) we have that $f * g \in D_{\rho}^{P}(G)$ whenever $f \in D_{\rho}^{p}(G)$ and $g \in D_{\rho}^{q}(G)$, and

$$
D_{\rho}^{r}(f * g)=D_{\rho}^{p} f * g=f * D_{\rho}^{q} g ;
$$

cf. Hewitt and Ross [1], (20.18).

It will appear in Theorem 3.1.3 that for any $L$ and any $\rho$ (as above) 


$$
L_{T}(G) \subset D_{\rho}^{L}(G)
$$

where $T$ is relatively compact; cf. Katznelson [1], Chapter VI, Section 4, Exercise 6. With this in mind we shall examine a derivative analogue of Bernstein's inequality for the space $L_{\Upsilon}(G)$.

\subsection{Bernstein's inequality for differentiation along a one-parameter subgroup}

Let $\rho$ be a continuous homomorphism of $R$ into $G$. To each $\gamma \in \Gamma$ there corresponds a unique $\zeta_{\gamma} \in R$, defined by

$$
\gamma(\rho(r))=\exp \left(i \zeta \gamma^{r}\right)
$$

(note that $\gamma \circ \rho$ is a continuous character of $R$ ). We then have:

3.1.1 LEMMA. The real-valued function $\Lambda$ on $\Gamma$, defined by

$$
\Lambda(\gamma)=\zeta_{\gamma},
$$

is continuous.

Proof. As $\Lambda$ is a homomorphism of $\Gamma$ into $R$ it suffices to prove that it is continuous at zero. In view of 1.2 .6 of Rudin [1] all we have to show is that given a compact set $C \subset R$ and $\varepsilon>0$,

$$
\sup _{r \in C}|\exp (i \Lambda(\gamma) r)-I|<\varepsilon
$$

for all $\gamma$ in some neighbourhood of zero.

Now (3.1.1) is equivalent to

$$
\sup _{r \in C}|\gamma(\rho(r))-1|<\varepsilon
$$

which is implied by

$$
\sup _{x \in \rho(C)}|\gamma(x)-1|<\varepsilon
$$

Since $\rho$ is continuous and $C \subset R$ is compact $\rho(C)$ must be compact in G; hence, by Rudin [1], 1.2 .6 again,

$$
\Omega=\left\{\gamma \in \Gamma: \sup _{x \in \rho(C)}|\gamma(x)-1|<\varepsilon\right\}
$$


is a neighbourhood of zero. Using (3.1.2) we see that (3.1.1) holds for all $\gamma \in \Omega$

As a preliminary result we require:

3.1.2 LEMMA. Let $\mathrm{T} \subset \mathrm{I}$ be relatively compact. Then we can find $k \in L^{I} \cap C_{0}(G)$ such that $\hat{k}=1$ on a neighbourhood of $T, \hat{k} \in C_{00}(\Gamma)$ and $k \in D_{\rho}^{I}(G)$. Furthermore $D_{\rho}^{I} k \in L^{I} \cap C_{0}(G)$ and $\left(D_{\rho}^{I} k\right)^{\wedge} \in C_{00}(\Gamma)$.

Proof. Let $\Omega$ be a relatively compact neighbourhood of $T$. Then, for any relatively compact non-void open set $\nabla, \Omega+\nabla-\nabla$ is relatively compact. Let $g, h$ be those elements of $L^{2}(G)$ having Fourier transforms $\xi_{\nabla}, \xi_{\Omega-\nabla}$ respectively and put $k=\theta(\nabla)^{-1} g h$. Furthermore define

$$
j=i \theta(\nabla)^{-1}(\phi \hbar+\psi g) \text {, }
$$

where $\phi, \psi \in L^{2}(G)$ are chosen having Fourier transforms $\Lambda \xi_{\nabla}, \Lambda \xi_{\Omega-\nabla}$ respectively (recall that by Lemma $3.1 .1, \Lambda$ is continuous). Then (cf. the proof of Theorem B.I.I) $k, j \in L^{I} \cap C_{0}(G), \hat{k}, \hat{j} \in C_{00}(\Gamma)$ and $\hat{k}=I$ on a neighbourhood of $T$.

$$
\begin{aligned}
& \text { Now (for } r \neq 0 \text { ) consider } \\
& \| r^{-1}\left(-p(r)^{k-k)}-j \|_{I}\right.
\end{aligned}
$$

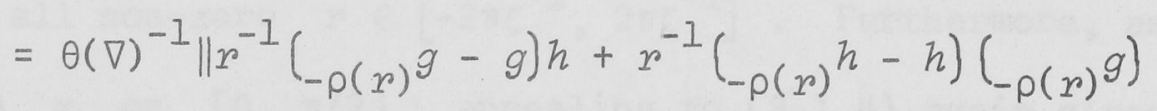

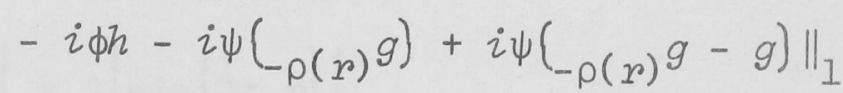

$$
\begin{aligned}
& \leq \theta(\nabla)^{-1}\left\{\left\|r^{-1}\left(_{-\rho(r)} g-g\right)-i \phi\right\|_{2}\|h\|_{2}\right. \\
& +\| r^{-1}\left(-\rho(r)^{h-h)}-i \psi\left\|_{2}\right\| g\left\|_{2}+\right\| \psi\left\|_{2}\right\|_{-\rho(x)^{g}}-g \|_{2}\right) \text {. }
\end{aligned}
$$

We shall prove that each of the terms in (3.1.3) tends to 0 in the limit as $r$ tends to 0 .

By Plancherel's theorem we have 


$$
\begin{aligned}
\left\|r^{-1}\left(-\rho(r)^{g}-g\right)-i \phi\right\|_{2} & =\|\left(r^{-1}\left(-\rho(r)^{g}-g\right)-i \phi \|_{2}\right. \\
& \left.=\iint_{\nabla}|\hat{g}(\gamma)|^{2}\left|r^{-1}(\gamma(\rho(r))-1)-i \hat{\phi}(\gamma)\right|^{2} d \gamma\right)^{\frac{1}{2}} \\
& \leq\|g\|_{2} \sup _{\gamma \in \nabla}\left|r^{-1}(\gamma(\rho(r))-1)-i \hat{\phi}(\gamma)\right| \\
& \leq\|g\|_{2} \sup _{2}\left\{\left|r^{-1}\left(\exp \left(i \zeta_{\gamma} r\right)-1\right)-i \zeta_{\gamma}\right|: \zeta_{\gamma} \in \Lambda\left(\nabla^{-}\right)\right\}
\end{aligned}
$$

If $\zeta_{\gamma} \neq 0$ then

$$
\begin{aligned}
& \left|r^{-1}\left(\exp \left(i \zeta_{\gamma} r\right)-1\right)-i \zeta_{\gamma}\right| \\
& =\left|r^{-1} \exp \left(i \frac{1}{2} \zeta \gamma^{r}\right)\left(\exp \left(i \frac{1}{2} \zeta \gamma^{r}\right\}-\exp \left(-i \frac{1}{2} \zeta^{r}\right)\right\}-i \zeta_{\gamma}\right| \\
& =\left|r^{-1} 2 \sin \left(\frac{1}{2} \zeta_{\gamma} r\right)-\zeta_{\gamma} \exp \left(-i \frac{1}{2} \zeta_{\gamma} r\right)\right| \\
& \leq\left|\zeta_{\gamma}\right|\left(\left|\left(\frac{1}{2} \zeta \gamma^{r}\right)^{-1} \sin \left(\frac{1}{2} \zeta_{\gamma} r\right)-1\right|+\left|1-\exp \left(-i \frac{1}{2} \zeta_{\gamma} r\right)\right|\right) \text {. }
\end{aligned}
$$

Now $\Lambda\left(\nabla^{-}\right)$is compact and hence we can find $\zeta>0$ such that

$$
\Lambda\left(\nabla^{-}\right) \subset[-\zeta, \zeta]
$$

Since $1-x^{-1} \sin (x)$ increases with $x$ on $(0, \pi]$, reference to $(3.1 .4)$ yields

$$
\left|\left(\frac{1}{2} \zeta \gamma^{r}\right)^{-1} \sin \left(\frac{1}{2} \zeta \gamma^{r}\right)-1\right| \leq\left|\left(\frac{1}{2} \zeta r\right)^{-1} \sin \left(\frac{1}{2} \zeta r\right)-1\right|
$$

for all non-zero $r \in\left[-2 \pi \zeta^{-1}, 2 \pi \zeta^{-1}\right]$. Furthermore, as $\sin (x)$ increases with $x$ on $[0, \pi / 2]$, appealing to (3.1.4) again gives

$$
\begin{aligned}
\left|1-\exp \left(-i \frac{1}{2} \zeta \gamma^{r}\right)\right| & =2\left|\sin \left(\frac{1}{4} \zeta \gamma^{r}\right)\right| \\
& \leq 2\left|\sin \left(\frac{1}{4} \zeta r\right)\right|
\end{aligned}
$$

for all $r \in\left[-2 \pi \zeta^{-1}, 2 \pi \zeta^{-I}\right]$. Hence

$$
\begin{aligned}
\sup \left\{\left|r^{-1}\left(\exp \left(i \zeta \gamma^{r}\right)-1\right)-i \zeta_{\gamma}\right|: \zeta_{\gamma}\right. & \left.\in \Lambda\left(\nabla^{-}\right)\right\} \\
& \leq|\zeta|\left\{\left|\left(\frac{1}{2} \zeta r\right)^{-1} \sin \left(\frac{1}{2} \zeta r\right)-1\right|+2\left|\sin \left(\frac{1}{4} \zeta r\right)\right|\right\}
\end{aligned}
$$


for all non-zero $r \in\left[-2 \pi \zeta^{-1}, 2 \pi \zeta^{-1}\right]$, and it follows that

$$
\lim _{r \rightarrow 0}\left\{\sup \left\{\left|r^{-1}\left(\exp \left(i \zeta_{\gamma} r\right)-1\right)-i \zeta_{\gamma}\right|: \zeta_{\gamma} \in \Lambda\left(\nabla^{-}\right)\right\}\right)=0 \text {. }
$$

Thus the first term in (3.1.3) tends to 0 as $r$ tends to 0 . The second term is treated similarly. For the third term see Hewitt and Ross [1], (20.4) and use the continuity of $\rho$.

It follows that $k \in D_{\rho}^{\perp}(G)$, and $D_{\rho}^{I_{k}}=j$ satisfies the hypotheses of the lemma. //

Using the notation of the proof of Lemma 3.1 .2 we obtain:

3.1.3 THEOREM. Let $T$ be a relatively compact subset of $\Gamma$ and suppose $f \in L_{T}(G)$. Then $D_{\rho}^{L} f$ exists and $D_{\rho}^{L}=j * f$.

Proof. For any $r \neq 0$ we have

$$
\begin{aligned}
& \left\|r^{-1}(-\rho(r) f-f)-j * f\right\|_{L}=\| r^{-1}\left(-\rho(r)^{k-k) * f-j * f \|_{L}}\right. \\
& \leq \| r^{-1}\left(-\rho(r)^{k-k)}-j\left\|_{I}\right\| f \|_{L}\right.
\end{aligned}
$$

which, by Lemma 3.1.2, tends to 0 as $r$ tends to 0 . Hence the result follows. //

3.1.4 COROLLARY. Suppose the hypotheses of Theorem 3.1 .3 are satisfied. Then

$$
\left\|D_{\rho}^{L} f\right\|_{L} \leq \kappa\|f\|_{L}
$$

where $k=\kappa(T, \Omega, \nabla)$ is independent of the choice of $f \in L_{T}(G)$.

3.1.5 REMARK. It is easily seen that once $\Omega$ and $\nabla$ (and hence $j$ ) have been chosen as in the proof of Lemma 3.1 .2 then Theorem 3.1 .3 holds for any (relatively compact) set $T \subset \Omega$.

3.1.6 REMARK. The constant $k$ in Corollary 3.1 .4 is given by

$$
\begin{aligned}
\kappa & =\|j\|_{1} \\
& \leq\left\{\frac{\theta(\Omega-\nabla)}{\theta(\nabla)}\right)^{\frac{1}{2}}\left\{\sup _{\gamma \in \Omega-\nabla}|\Lambda(\gamma)|+\sup _{\gamma \in \nabla}|\Lambda(\gamma)|\right\}
\end{aligned}
$$


(although this is not necessarily the best possible). Alternatively, once we know that $f \in L_{T}(G)$ is L-differentiable along $\rho$, then the definition of $D_{\rho}^{L} f$ gives us

$$
\left\|D_{\rho}^{L} f\right\|_{L} \leq \lim \inf _{r \rightarrow 0}\left(|r|^{-1} B_{T}^{L}(\rho(r))\right)\|f\|_{L}
$$

Corollary 3.1 .4 with $k$ as in $(3.1 .5)$ now follows readily from Theorem 1.1 .2$.

\subsection{A converse result}

In this section we consider a converse result for the derivative form of Bernstein's inequality obtained in 3.1. As a preliminary result we require:

3.2.1 LEMMA. Let $\gamma \in \Gamma$ and $\varepsilon>0$ be given. Then we can find $k \in L^{I} \cap D_{\rho}^{1}(G)$ such that $\|k\|_{1}=1, \hat{k}(\gamma)=1$ and

$$
\left\|D_{\rho}^{1} k-i \zeta_{\gamma} k\right\|_{1}<\varepsilon
$$

Proof. Choose $\nabla$ to be a relatively compact open symmetric neighbourhood of zero in $\Gamma$ such that for all $x \in \nabla$

$$
\left|\zeta_{\chi}\right|<\varepsilon / 2
$$

We now follow the proof of Lemma 3.1 .2 with $\nabla$ as above and $\Omega$ replaced by $\gamma$ to select $k \in L^{I} \cap C_{0}(G)$ such that $\|k\|_{1}=1, \hat{k}(\gamma)=1$ and $k \in D_{\rho}^{I}(G)$

To prove that $(3.2 .1)$ holds for this choice of $k$ we observe that

$$
\begin{aligned}
\left\|D_{\rho}^{I} k-i \zeta_{\gamma} k\right\|_{1} & =\theta(\nabla)^{-1}\left\|\phi h+g\left(\psi-\zeta_{\gamma} h\right)\right\|_{1} \\
& \leq \theta(\nabla)^{-1}\left(\|\phi\|_{2}\|h\|_{2}+\|g\|_{2}\left\|\psi-\zeta_{\gamma} h\right\|_{2}\right) .
\end{aligned}
$$


Now $\|g\|_{2}=\|h\|_{2}=\theta(\nabla)^{\frac{1}{2}}$. Furthermore, using $(3.2 .2)$,

$$
\begin{aligned}
\|\phi\|_{2} & \left.=\iint_{\nabla}\left|\zeta_{x}\right|^{2} d x\right)^{\frac{1}{2}} \\
& \leq \varepsilon / 2 \theta(\nabla)^{\frac{1}{2}}
\end{aligned}
$$

and

$$
\begin{aligned}
\left\|\psi-\zeta_{\gamma} h\right\|_{2} & \left.=\iint_{\gamma+\nabla}\left|\zeta_{x}-\zeta_{\gamma}\right|^{2} d x\right)^{\frac{1}{2}} \\
& \left.=\iint_{\gamma+\nabla}\left|\zeta_{x-\gamma}\right|^{2} d x\right)^{\frac{1}{2}} \\
& \leq \varepsilon / 2 \theta(\nabla)^{\frac{1}{2}} .
\end{aligned}
$$

These estimates combine with $(3.2 .3)$ to give

$$
\left\|D_{\rho}^{I} k-i \zeta_{\gamma} k\right\|_{1} \leq \varepsilon
$$

as required. //

The converse result we obtain will be in terms of a family $\left\{\rho_{\iota}\right\}_{\iota \in I}$ of continuous homomorphisms of $\mathrm{R}$ into $G$. It is to be expected that any result in this direction would, to a certain extent, depend on how "large" in $G$ is the subgroup generated by $\underset{\imath \in I}{U} \rho_{\ell}(R)$. We describe this

"largeness" as follows:

To each $\iota \in I$ will correspond a map $\Lambda_{\iota}$ (as in Lemma 3.1.1) where

$$
\Lambda_{\tau}(\gamma)=\zeta_{\gamma}^{\imath} \quad(\gamma \in \Gamma)
$$

We shall say that (the family) $\left\{\rho_{\ell}\right\}_{\iota \in I}$ is jointly-proper if $T \subset \Gamma$ is relatively compact whenever $\Lambda_{\iota}(T)$ is relatively compact in $R$ for all $\iota \in I$; $е f$. Bourbaki [1], I, Section 10.3, Proposition 7. Consider the following statements:

(a) $\left\{\rho_{\iota}\right\}_{\iota \in I}$ is jointly-proper; 
55

(b) $\Xi \subset \Gamma$ has the property that for every $\imath \in I$ there exists $\kappa_{l}>0$ such that if $\gamma \in \Xi$ and $\Omega$ is a relatively compact open neighbourhood of $\gamma$, then there exists $f_{\gamma} \in L_{\Omega} \cap D_{\rho_{\ell}}^{L}(G)$ with $\gamma \in \Sigma(f)$ and

$$
\left\|D_{\rho_{\imath}}^{L} f_{\gamma}\right\|_{L} \leq \kappa_{\imath}\left\|f_{\gamma}\right\|_{L}
$$

(c) $\Xi$ is relatively compact.

Our converse result is then:

3.2.2 THEOREM. If (a) holds then (b) $\Rightarrow$ (c) for all $\Xi \subset \Gamma$.

Proof. Suppose $\Xi$ is a non-void subset of $\Gamma$ with the property stated in (b). Choose $\gamma \in \Xi$ and $i \in I$, and take $k_{\imath}$ as in Lemma 3.2.1 satisfying $(3.2 .1)$ with $\rho=\rho_{\imath}$ and $\varepsilon=1 / 2$. Since $\hat{k}_{\imath}(\gamma)=1$ we can appeal to Lemma 2.I.I to deduce the existence of $l_{\imath} \in L^{I}(G)$ such that $\hat{k}_{\imath} \hat{\imath}_{\imath}=1$ on some relatively compact open neighbourhood $\Omega_{\imath}$ of $\gamma$ and $\left\|I_{l}\right\|_{I}<2$.

Now, by assumption, there exists $f_{\gamma} \in L_{\Omega_{\tau}} \cap D_{\rho_{\tau}}^{L}(G)$ with $\gamma \in \Sigma\left(f_{\gamma}\right)$ and

$$
\left\|D_{\rho_{\imath}}^{L} f_{\gamma}\right\|_{L} \leq \kappa_{\iota}\left\|f_{\gamma}\right\|_{L}
$$

By the choice of $k_{\imath}$ we have that

$$
\left\|\left(D_{\rho_{L}}^{I} k_{\iota}-i \zeta_{\gamma}^{c^{k}}\right) * f_{\gamma}\right\|_{L} \leq \frac{1}{2}\left\|f_{\gamma}\right\|_{L}
$$

and (using $(3.0 .1)$ )

$$
\begin{aligned}
\left|\zeta_{\gamma}^{\iota}\right|\left\|k_{\tau} * f_{\gamma}\right\|_{L} & \leq\left\|k_{\tau} * D_{\rho_{\tau}}^{L} f_{\gamma}\right\|_{L}+\frac{1}{2}\left\|f_{\gamma}\right\|_{L} \\
& \leq\left(k_{\tau}+1 / 2\right)\left\|f_{\gamma}\right\|_{L},
\end{aligned}
$$

the latter step using (b). But

$$
f_{\gamma}=k_{\iota} * z_{\iota} * f_{\gamma}
$$


and so

$$
\begin{aligned}
\left\|f_{\gamma}\right\|_{L} & \leq\left\|z_{\iota}\right\|_{I}\left\|k_{\tau} * f_{\gamma}\right\|_{L} \\
& \leq 2\left\|k_{L} * f_{\gamma}\right\|_{L} .
\end{aligned}
$$

This, combined with $(3.2 .4)$ and the fact that $k_{\ell} * f_{\gamma} \neq 0$, gives

$$
\left|\zeta_{\gamma}^{\iota}\right| \leq 2 \kappa_{\iota}+1
$$

This means that $\Lambda_{\iota}(\Xi)$ is relatively compact in $R$ for each $\imath \in I$ and hence, as $\left\{\rho_{\imath}\right\}_{\iota \in I}$ is assumed to be jointly-proper, $\Xi$ must be relatively compact. //

As a converse to Theorem 3.2 .2 we also have:

3.2.3 THEOREM. If there exists $p \in[1, \infty]$ such that (b) $\Rightarrow$ (c) (with $L(G)=L^{p}(G)$ ) for all $\Xi \subset \Gamma$ then (a) holds.

Proof. Suppose that $T$ is a non-relatively compact subset of $\Gamma$ for which each $\Lambda_{\ell}(T)$ is relatively compact in $R$. Without loss of generality we can assume that $T$ is closed.

For every $\imath \in I$ choose a corresponding positive $k_{\imath} \in R$ such that

$$
\Lambda_{\imath}(T) \subset\left(-\frac{1}{2} K_{\imath}, \frac{1}{2} K_{\iota}\right)
$$

and let $\nabla_{i}$ be any symmetric relatively compact open neighbourhood of zero in $\Gamma$ satisfying

$$
\Lambda_{\imath}\left(2 \nabla_{\imath}\right) \subset\left(-\frac{1}{2} K_{\imath}, \frac{1}{2} K_{\imath}\right)
$$

Now, given $\gamma \in T$, we can find a relatively compact open meighbourhood $\Omega_{\imath}(\gamma)$ of $\gamma$ such that $\Omega_{\imath}(\gamma) \subset \gamma+\nabla_{\imath}$ and

$$
\theta\left(\Omega_{\imath}(\gamma)-\nabla_{\imath}\right) \leq 2 \theta\left(\nabla_{\imath}\right)
$$

Put

$$
j_{\imath}=i \theta\left(\nabla_{\imath}\right)^{-1}\left(\phi_{\imath} h_{\imath}+\psi_{\imath} g_{\imath}\right)
$$


where $g_{\imath}, h_{\imath}, \phi_{\imath}, \psi_{\imath}$ in $L^{2}(G)$ are chosen as in the proof of Lemma 3.1 .2 (with $\Omega=\Omega_{\imath}(\gamma)$ and $\left.\nabla=\nabla_{\imath}\right)$. Then, using $(3.2 .5),(3.2 .6)$ and the definition of $\Omega_{\imath}(\gamma)$, we have

$$
\begin{aligned}
\left\|j_{\imath}\right\|_{I} & \leq \theta\left(\nabla_{\imath}\right)^{-1}\left(\left\|\phi_{\iota}\right\|_{2}\left\|h_{\imath}\right\|_{2}+\left\|\psi_{\imath}\right\|_{2}\left\|g_{\imath}\right\|_{2}\right) \\
& \leq \theta\left(\nabla_{\iota}\right)^{-1} 2 \kappa_{\imath} \theta\left(\nabla_{\imath}\right)^{\frac{1}{2}} \theta\left(\Omega_{\iota}(\gamma)-\nabla_{\imath}\right)^{\frac{1}{2}} \\
& \leq 2 \sqrt{2} \kappa_{\imath} .
\end{aligned}
$$

Now, if $\Omega$ is any relatively compact open neighbourhood of $\gamma$, we can

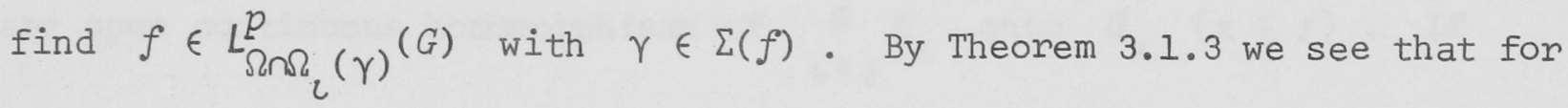
all $\imath \in I, f \in D_{\rho_{\imath}}^{p}(G)$ and (making use of Remark 3.1.5) $D_{\rho_{\imath}}^{p} f=j_{\imath} * f$. By $(3.2 .7)$

$$
\begin{aligned}
\left\|D_{\rho_{\imath}}^{p} f\right\|_{p} & =\left\|j j_{\imath} * f\right\|_{p} \\
& \leq 2 \sqrt{2} \kappa_{\imath}\|f\|_{p} .
\end{aligned}
$$

Thus $T$ has the property stated in (b) but is not relatively compact, and the result follows.

\subsection{A necessary and sufficient condition for certain $\left\{\rho_{2}\right\}_{\imath \in I}$ to be jointly-proper}

The remainder of this chapter is spent considering the significance of certain $\left\{\rho_{\iota}\right\}_{\iota \in I}$ being jointly-proper. If we assume that $\left\{\rho_{\iota}\right\}_{\iota \in I}$ is "independent" in the sense of (3.3.2) and that for any finite set $J \subset I$, $\sum_{\imath \in J} \rho_{\imath}(R)^{-}$is closed in $G$ then it appears that $\left\{\rho_{\imath}(R)\right\}_{\imath \in I}$ is jointlyproper if and only if the smallest closed subgroup of $G$ containing

$\cup \rho_{\iota}(R)$ has a decomposition into a product of the form $\mathrm{R}^{m} \times \mathrm{T}^{n} \times D$ 
where $m, n$ are non-negative integers and $D$ is a discrete group. We shall require some preliminary results on Cartesian products of locally compact groups.

Let $\left\{G_{\imath}\right\}_{\iota \in I}$ be a family of topological groups. Then $\underset{\imath \in I}{P} G_{\imath}$ (respectively $\underset{\imath \in I}{P *} G_{\imath}$ ) will denote the direct product (respectively the weak direct product) of the $G_{\imath}$. The projections (see Hewitt and Ross [1], $(6.8))$

$$
\pi_{x}: \underset{\imath \in I}{P} G_{\imath} \rightarrow G_{x}
$$

are open continuous homomorphisms of $\underset{\imath \in I}{P} G_{\ell}$ onto $G_{x}(x \in I)$. If $x \in G_{x}$ then $\left.{ }^{(}\right)_{x}$ will denote that element of $\underset{\imath \in \in_{I}}{P} G_{\mathcal{C}}$ such that $\pi_{x}\left((x)_{x}\right)=x$ and $\pi_{\imath}\left((x)_{x}\right)=0$ for $\imath \neq x$.

For the topological groups $G, G^{\prime}$ we shall write $G \stackrel{\text { tep }}{=} G^{\prime}$ if there is a mapping (called a topological isomorphism) of $G$ onto $G^{\prime}$ which is both a group isomorphism and a homeomorphism. We shall be making frequent use of the following result:

3.3.1 THEOREM. Let $G$ be a compact Hausdorff group and suppose $\left\{G_{\imath}\right\}_{\imath \in I}$ is a family of closed subgroups of $G$ satisfying:

The smallest closed subgroup of $G$ containing all the $G_{C}$ is $G$ itself;

$$
\bigcap_{\imath \in I} G^{(\imath)}=\{0\},
$$

where $G^{(\imath)}$ denotes the smallest closed subgroup of $G$ containing $\underset{\substack{x \in I \\ x \neq \imath}}{U} G_{x}$. Then $\underset{\imath \in I}{P} G_{l} \stackrel{\text { top }}{=} G$. Furthermore there is a topological isomorphism $n$ of $\underset{\imath \in I}{P} G_{\imath}$ onto $G$ such that 


$$
n\left((x)_{2}\right)=x
$$

for alt $\iota \in I$ and $x \in G_{\imath}$.

Proof. This is proved in Pontrjagin [1], Section 21.

Taking $G_{l}=\rho_{l}(R)^{-}$in Theorem 3.3 .1 we obtain:

3.3.2 COROLLARY. Let $H$ denote the smaZlest closed subgroup of $G$ containing $U_{L \in I} \rho_{\mathcal{L}}(\mathrm{R})$. Suppose that $\left\{\rho_{\mathcal{L}}(\mathrm{R})\right\}_{L \in I}$ satisfies $(3.3 .2)$ and that for each finite set $J \subset I, \sum_{l \in J} \rho_{l}(R)^{-}$is closed in $G$. Then

(a) $\rho_{\imath}$ is a topological isomorphism of $R$ onto $\rho_{\imath}(R)$ for finitely mony $i \in I$, and otherwise $\rho_{i}(R)^{-}$is compact;

(b) there is a topological isomorphism $n$ of $\mathrm{P}_{\imath \in I} \rho_{\imath}\left(\mathrm{R}^{-}\right.$onto $H$ such that for each $\imath \in \mathbb{R}$ and $x \in \rho_{\imath}(R)^{-}$,

$$
\eta\left((x)_{2}\right)=x \text {. }
$$

Proof. (a) Suppose $\rho_{\tau_{n}}$ is a topological isomorphism of $R$ onto $\rho_{c_{n}}(R)$ for $n \in\{1,2, \ldots, m\}$. Then each $\rho_{c_{n}}(R)$ is closed in $G$ (see Hewitt and Ross [1], (5.40) (d)) and so, by assumption, $\sum_{n=1}^{m} \rho_{c_{n}}(\mathrm{R})$ is closed. Since $\left\{\rho_{\imath}(R)\right\}_{\imath \in I}$ satisfies (3.3.2) it follows from Hewitt and Ross [1], (6.12) that

$$
\sum_{n=1}^{m} \rho_{c_{n}}(\mathrm{R}) \stackrel{\text { top }}{\rightleftharpoons} \stackrel{P}{\mathrm{P}}_{n=1}^{m} \rho_{c_{n}}(\mathrm{R})
$$

The first part of (a) now follows from Hewitt and Ross [1], (9.14) and the remainder from Hewitt and Ross [1], (9.1).

(b) This follows from (a), the proof of Hewitt and Ross [1], (6.12) and Theorem 3.3.1.

Given $\iota \in I, \Gamma_{\imath}$ will denote the character group of the Hausdorff 
locally compact Abelian group $\rho_{\imath}(R)^{-}$(with the relative topology induced by $G$ ). To each $\Gamma_{\iota}$ will correspond a map $\Lambda_{\iota}^{\prime}$ (as in the preamble to Theorem 3.2.2). Suppose we have that $\left\{\rho_{\imath}\right\}_{\imath \in I}$ and $\eta$ satisfy the conditions of corollary 3.3.2. Since $\eta$ is a topological isomorphism of $\underbrace{}_{\iota \in I} \rho_{\iota}(R)^{-}$onto $H$ its adjoint $\eta^{\sim}$ is a topological isomorphism of $\Gamma_{H}$ onto $\Gamma^{\prime}\left(\Gamma_{H}, \Gamma^{\prime}\right.$ are the character groups of $H, \underset{\imath \in I}{P} \rho_{\ell}(R)^{-}$

respectively); see Hewitt and Ross [1], (24.37) and (24.41) (c). Furthermore it appears (using Hewitt and Ross [1], (23.18) and (23.21)) that

$$
\Gamma^{\prime} \stackrel{\text { top }}{\cong} \underset{\imath \in J}{\mathrm{P}} \Gamma_{\imath} \times \underset{\imath \in I \backslash J}{\mathrm{P} *} \Gamma_{\imath}
$$

where $J \subset I$ is the finite set of indices $\iota$ for which $R \stackrel{\text { top }}{=} \rho_{\imath}(R)$ and $\underset{\imath \in I \backslash J}{\mathrm{P} *} \Gamma_{\imath}$ is given the discrete topology. Every element of $\Gamma^{\prime}$ is of the form $\left[r_{\imath}\right]$, where

$$
\left[\gamma_{\imath}\right]\left(\left(x_{\imath}\right)\right)=\prod_{\imath \in I} \gamma_{\iota}\left(x_{\imath}\right)
$$

for any $\left(x_{\imath}\right) \in \underset{\imath \in I}{P} \rho_{\imath}(R)^{-}$(note that only a finite number of the terms in the product differ from 1 ). The projections $\pi_{x}^{*}$, defined by

$$
\pi_{x}^{*}\left(\left[\gamma_{\imath}\right]\right)=\gamma_{x}
$$

are continuous homomorphisms of $\Gamma^{\prime}$ onto $\Gamma_{X^{*}}$. We can relate the maps $\Lambda_{\zeta}$, $\Lambda_{\ell}^{\prime}$ as follows:

3.3.3 LEMMA. Suppose $\left\{p_{\imath}\right\}_{\imath \in I}, H$ and $n$ satisfy the conditions of Corolzary 3.3.2. Then, for any $T \subset \Gamma$ and $\tau \in I$, we have

$$
\Lambda_{\tau}(T)=\Lambda_{\imath}^{p}\left(\pi_{\imath}^{*}\left(\eta^{\sim}\left(\left.T\right|_{H}\right)\right\}\right) \text {, }
$$

where $\left.\mathrm{T}\right|_{H}$ denotes the restriction of $\mathrm{T}$ to $H$.

Proof. Choose $\gamma \in T$ and put 


$$
\eta^{\sim}\left(\left.\gamma\right|_{H}\right)=[\gamma]
$$

(note that $\left.\gamma\right|_{H} \in \Gamma_{H}$ ). Then, for any $\imath \in I$ and $r \in R$,

$$
\begin{aligned}
& \gamma\left(\rho_{\imath}(r)\right)=\left(\left.\gamma\right|_{H}\right)\left(\rho_{\imath}(r)\right) \\
& =\left\{\eta^{\sim-1}\left(\left[\gamma_{\imath}\right]\right)\right\}\left(\rho_{\imath}(r)\right) \\
& =\left[\gamma_{\iota}\right]\left\{\eta^{-1}\left(\rho_{\iota}(r)\right)\right\} \\
& =\left[\gamma_{\imath}\right]\left(\left(\rho_{\ell}(r)\right)_{\imath}\right) \\
& =\gamma_{\imath}\left(\rho_{\imath}(r)\right) \\
& =\pi_{\tau}^{*}\left(\eta^{\sim}\left(\left.\gamma\right|_{H}\right)\right)\left(\rho_{\imath}(x)\right) \text {, }
\end{aligned}
$$

that is,

$$
\Lambda_{\iota}(\gamma)=\Lambda_{\imath}^{\prime}\left(\pi *\left(n^{\sim}\left(\left.\gamma\right|_{H}\right)\right)\right) \cdot \quad / /
$$

Before proving the main result of this section we consider a condition on $\left\{\rho_{\imath}\right\}_{\imath \in I}$ which (see Lemma 3.3.5) turns out to be weaker than $\left\{\rho_{\ell}\right\}_{\imath \in I}$ being jointly-proper.

3.3.4 THEOREM. Suppose that $\left\{\rho_{\imath}\right\}_{\imath \in I}$ and $H$ satisfy the conditions of Corolzary 3.3.2. Then the folzowing are equivalent:

(a) if $U_{\imath \in I} \rho_{\imath}(R) \subset A(G, \Delta)$ for some $\Delta \subset \Gamma$ then $\Delta$ is relatively compact;

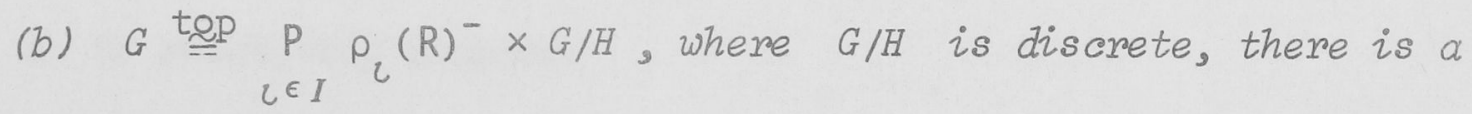
finite set $J \subset I$ such that for $\imath \in J$.

$$
R \stackrel{\text { top }}{\cong} \rho_{\imath}(R)=\rho_{\imath}(R)^{-}
$$

and otherwise $\rho_{\tau}(R)^{-}$is compact;

(c) $\lambda(H)>0$.

Proof. (a) $\Rightarrow(b)$ Since $H$ is a closed subgroup of $G$ we have that

$$
H=A(G,(A(\Gamma, H))\}
$$


and, by assumption, $A(\Gamma, H)$ must be relatively compact. But $A(\Gamma, H)$ is also a closed subgroup of $\Gamma$, whence it follows that $H$ is open. We also have, using Hewitt and Ross [1], (25.20) and (24.24), that $H$ is connected and divisible. Hence, by Corollary 3.3.2 (b) and Hewitt and Ross [1], $(6.22)(b)$,

$$
G \stackrel{t o p}{=} P_{\iota \in I} \rho_{\imath}(\mathrm{R})^{-} \times G / H
$$

The remainder of the conclusion follows from the fact that $H$ is open and from Corollary 3.3 .2 (a).

$(b) \Rightarrow(c)$. Since $G / H$ is a discrete group it follows that $H$ is open.

(c) $\Rightarrow(a)$. Suppose

$$
\bigcup_{\imath \in I} \rho_{l}(R) \subset A(G, \Delta)
$$

for some $\Delta \subset \Gamma$. Then

$$
H \subset \mathrm{A}(G, \Delta)
$$

and, since $\lambda(H)>0$, it follows (see Hewitt and Ross [1], (23.24)) that $\Delta$ is relatively compact, as required. //

3.3.5 LEMMA. Suppose $\left\{\rho_{\tau}\right\}_{\tau \epsilon_{I}}$ is jointly-proper. If $\Delta \subset \Gamma$ has the property that

$$
\underset{\imath \in I}{U} \rho_{\imath}(R) \subset A(G, \Delta)
$$

then $\Delta$ is relatively compact.

Proof. Suppose that

$$
\bigcup_{\imath \in I} \rho_{\ell}(R) \subset A(G, \Delta),
$$

where $\Delta \subset \Gamma$. Then, for any $\gamma \in \Delta, r \in R$ and $\iota \in I$,

$$
\gamma\left(\rho_{\imath}(r)\right)=2 \text {, }
$$

that is, $\underset{\iota \epsilon_{I}}{U} \Delta_{\iota}(\Delta)=\{0\}$. Since $\left\{\rho_{\iota}(R)\right\}_{\iota \epsilon_{I}}$ is assumed to be jointlyproper it follows that $\Delta$ is relatively compact. // 
Making use of Theorem 3.3 .4 and Lemma 3.3 .5 we have:

3.3.6 THEOREM. Suppose $\left\{\rho_{\imath}\right\}_{\iota \in_{I}}, \eta$ and $H$ satisfy the conditions of Corollary 3.3.2. Then the following are equivalent:

(a) $\left\{\rho_{\imath}\right\}_{\imath \in I}$ is jointly-proper;

(b) $G \stackrel{\text { top }}{\Rightarrow} \underset{l \in I}{P} \rho_{\imath}(R) \times G / H$, where $G / H$ is discrete, all but a finite number of the $\rho_{i}$ are trivial, and otherwise either $\rho_{i}(R) \stackrel{\operatorname{top}}{=} R$ or $\rho_{i}(R) \stackrel{\text { top }}{=} T$.

Proof. $(a) \Rightarrow(b)$. We know from Lemma 3.3 .5 and Theorem 3.3.4 that

$$
G \stackrel{t o p}{\cong} \underset{\imath \in I}{P} \rho_{\iota}(R)^{-} \times G / H
$$

where $G / H$ is discrete and $\rho_{\imath}(R)=\rho_{\imath}(R)^{-} \stackrel{t e p}{=} R$ for only finitely many $i \in I$. Suppose that $x \in I$ and $\{0\} \neq \rho_{x}(R)^{-}$is compact. Put

$$
\Upsilon_{n}^{\prime \prime}=\left\{[\gamma]_{x} \in \Gamma^{\prime}: \Lambda_{x}^{\prime}(\gamma) \subset[-n, n]\right\},
$$

where $n \in\{1,2, \ldots\}, \gamma \in \Gamma_{x}$ and $[\gamma]_{x}$ corresponds to $(\gamma)_{x} \in \underset{\iota \in I}{P *} \Gamma_{\imath}$ as in the comments preceding Lemma 3.3.3. Let

$$
\tau_{n}^{\prime}=n^{-1}\left(\Upsilon_{n}^{\prime \prime}\right)
$$

and choose any set $T_{n} \subset \Gamma$ such that

$$
\left.\mathrm{T}_{n}\right|_{H}=\mathrm{T}_{n}^{\prime}
$$

(the existence of such a set $T_{n}$ is guaranteed by Hewitt and Ross [1], (24.11)). For any $i \in I$ it follows from Lemma 3.3.3 that

$$
\begin{aligned}
\Lambda_{\iota}\left(T_{n}\right) & =\Lambda_{\iota}^{\prime}\left(\pi_{\imath}^{*}\left(\eta^{\sim}\left(\left.T_{n}\right|_{H}\right\}\right)\right) \\
& = \begin{cases}\{0\} & (\iota \neq x) \\
\Lambda_{x}^{\prime}\left(\Gamma_{x}\right) \cap[-n, n] & (\iota=x) .\end{cases}
\end{aligned}
$$

Thus $\underset{\imath \in I}{U} \Lambda_{\iota}\left(T_{n}\right)$ is relatively compact in $R$ and, by assumption, $T_{n}$ is 
relatively compact for each $n \in\{1,2, \ldots\}$. Now, by Hewitt and Ross [1], (24.5), the restriction map

$$
\sigma:\left.\Gamma \rightarrow \Gamma\right|_{H}
$$

is continuous (note that Lemma 3.3 .5 and Theorem 3.3 .4 combine with (a) to show that $H$ is open). From this, the continuity of $\eta^{\sim}$ and $\pi_{x}^{*}$, and the fact that $\Gamma_{x}$ is discrete we see that

$$
\pi_{x}^{*}\left(n^{\sim}\left\{\sigma\left(\Gamma_{n}\right) !\right)=\left\{\gamma \in \Gamma_{x}: \Lambda_{x}^{\prime}(\gamma) \subset[-n, n]\right\}\right.
$$

is finite for all $n \in\{1,2, \ldots\}$. But $\Lambda_{x}^{\prime}\left(\Gamma_{x}\right)$ is a subgroup of $R$, whence it follows that for some $r \in R$,

$$
\Lambda_{x}^{\prime}\left(\Gamma_{x}\right) \cong r z \text {. }
$$

As we have assumed that $\rho_{x}(R)^{-}$is non-zero we obtain

$$
\Lambda_{x}^{\prime}\left(\Gamma_{x}\right) \cong Z
$$

But clearly $\Lambda_{x}^{\prime}$ is one-to-one on $\Gamma_{x}$ and so

$$
\Gamma_{x} \cong z
$$

that is,

$$
\rho_{x}(R)^{- \text {top }} \stackrel{T}{=} T \text {. }
$$

Let $U$ denote a topological isomorphism of $\rho_{x}(R)^{-}$onto $T$. Since $U \circ \rho_{x}$ is a non-trivial continuous character of $R$ it follows that

$$
u\left(\rho_{x}(R)\right)=T
$$

$\left(U\left(\rho_{x}(R)\right)\right.$ is a non-zero connected subgroup of $\left.T\right)$ and hence

$$
\rho_{x}(R) \stackrel{\text { top }}{=} T
$$

Now put

$$
J=\left\{c \in I: \rho_{\imath}(R) \stackrel{\text { top }}{=} T\right\} .
$$

For each $\imath \in J$ choose $\gamma_{\imath} \in \Gamma_{\imath}$ and set

$$
T=\left\{\left[Y_{\imath}\right]_{\iota}: \iota \in J\right\}
$$


Then, using Lernma 3.3 .3 ,

$$
\Lambda_{\imath}(T)= \begin{cases}\Lambda_{\imath}^{\prime}\left(\gamma_{\iota}\right) & (\iota \in J) \\ \{0\} & (\iota \notin J) .\end{cases}
$$

Hence each $\Lambda_{\iota}(T)$ is relatively compact in $R$ and, by $(a)$, $T$ must be relatively compact. However $\underset{\imath \in J}{P *} \Gamma_{\imath}$ is discrete; so $T$ and thus $J$ must be finite.

$(b) \Rightarrow(a)$. Suppose $T$ is a non-relatively compact subset of $T$ for which each $\Lambda_{\imath}(T)$ is relatively compact in $R$. We shall derive a contradiction.

By Theorem 3.3 .4 and its proof it is clear that $H$ is an open divisible subgroup of $G$. Furthermore the proofs of (6.22) and (6.11) of Hewitt and Ross [1] show that we can choose a topological isomorphism $U$ of $G$ onto $H \times G / H$ such that for all $x \in H$,

$$
U(x)=(x, 0) \text {. }
$$

Now $U^{\sim}$ is a topological isomorphism of $\Gamma_{H \times G / H}$ onto $\Gamma$. Hence for any $\gamma \in \Gamma$ we have

$$
u^{\sim-1}(\gamma)=\left[\gamma_{1}, \gamma_{2}\right]
$$

where $\gamma_{I} \in \Gamma_{H}$ and $\gamma_{2} \in \Gamma_{G / H}$, and for $x \in H$

$$
\begin{aligned}
\gamma(x) & =u^{\sim}\left(\left[\gamma_{1}, \gamma_{2}\right]\right)(x) \\
& =\left[\gamma_{1}, \gamma_{2}\right](u(x)) \\
& =\gamma_{1}(x),
\end{aligned}
$$

that is,

$$
\left.\gamma\right|_{H}=\gamma_{1}
$$

Now, since $\Upsilon$ is assumed not to be relatively compact, neither is $U^{-1}(T)$. Using Tychonoff's theorem and the compactness of $\Gamma_{G / H}$ it 
follows that

$$
\left.T\right|_{H}=\pi_{1}\left(U^{\sim-1}(T)\right)
$$

is not relatively compact in $\Gamma_{H}$. Since, by assumption, $\rho_{\ell}(R)$ is only non-zero for finitely many $\iota \in I$ we can appeal once more to Tychonoff's theorem to deduce the existence of $x \in I$ for which $\rho_{x}(R)$ is non-zero and

$$
T_{x}=\pi_{x}^{*}\left(\eta^{\sim}\left(\left.T\right|_{H}\right)\right)
$$

is not relatively compact in $\Gamma_{x}$. We note however that by Lemma 3.3 .3 and our assumption on $\mathrm{T}, \Lambda_{x}^{\prime}\left(\mathrm{T}_{x}\right)$ is relatively compact in $R$.

Let $\lambda_{x}$ denote a Haar measure on $\rho_{x}(R)$. Given $\nabla$, a relatively compact open neighbourhood of zero in $\Gamma_{x}$, the non-relative compactness of $T_{x}$ ensures the existence of $\left\{\gamma_{m}\right\}_{m=1}^{\infty} \subset T_{x}$ such that

$$
\left(\gamma_{m}+\nabla\right) \cap\left(\gamma_{m^{\prime}}+\nabla\right)=\square \text { for } m \neq m^{\prime}
$$

However $\left\{\Lambda_{x}^{\prime}\left(\gamma_{m}\right)\right\}_{m=1}^{\infty}$ is relatively compact in $R$ (see the previous paragraph) and hence there exists a subsequence $\left\{\Lambda_{x}^{\prime}\left(\gamma_{m_{n}}\right)\right\}_{n=1}^{\infty}$ which is Cauchy in $R$. Choose any compact set $C \subset R$ such that $\lambda_{x}\left(\rho_{x}(C)\right)>0$. We can find $n_{0}$ such that for all $n \geq n_{0}$ and $r \in C$

$$
\left|\exp \left(i \Lambda_{x}^{\prime}\left(\gamma_{m_{n}}\right) r\right)-\exp \left(i \Lambda_{x}^{\prime}\left(\gamma_{m_{n_{0}}}\right) r\right)\right|<1 / 2
$$

that is, for all $n \geq n_{0}$ and $x \in \rho_{x}(C)$

$$
\left|\gamma_{m_{n}}(x)-\gamma_{m_{n_{0}}}(x)\right|<1 / 2
$$

Since $\lambda_{x}\left(\rho_{x}(C)\right)>0$ we can appeal to Hewitt and Ross [1], (23.16) to deduce that $\left\{\gamma_{m_{n}}\right\}_{n=n_{0}}^{\infty}$ (which, by (3.3.3), is closed) is compact. However this is impossible since (3.3.3) also shows that $\left\{\gamma_{m_{n}}+\nabla\right\}_{n=n_{0}}^{\infty}$ is an open cover which cannot be reduced to a finite subcover. Thus no such $T$ exists and the theorem is proved. 
APPENDIX A

\section{SOME RESULTS ON THE FOURIER TRANSFORM AND}

\section{SPECTRUM OF AN $L^{p}$-FUNCTION}

In this appendix we shall be concerned with some results on the Fourier transform of an $L^{p}$-function $(p \in[1, \infty])$, where the underlying group is assumed to be Abelian. These (see Theorem A.1.2) include some standard properties of the Fourier transform of an $L^{l}$-function. We shall also prove the equivalence of the notions of the spectrum of an $\quad L^{p}$-function and the support of its Fourier transform.

\section{A.1 Some properties of the Fourier transform}

Before proving one of the main properties of the Fourier transform we need to demonstrate the measurability of the frequently used function

$$
F:(x, y) \rightarrow f(x-y),
$$

where $f$ is $\lambda$-measurable. This problem is usually avoided by assuming $f$ to be Borel measurable ( $c f$. Hewitt and Ross [1], Volume I, p. 264).

A.1.1 LEMMA. Suppose $f$ is $\lambda$-measurable on $G$. Then $F$ defined as in (A.I.I) is $\lambda \times \lambda$-measurable.

Proof. Initially we prove the result for $f \in L^{l}(G)$, and then extend it to arbitrary measurable $f$.

Let $N$ be a negligible subset of $G$ and choose a sequence $\left(V_{n}\right)$ of open sets such that

$$
\lambda\left(V_{n}\right)<n^{-1} \text { and } N \subset V_{n} \quad(n=1,2, \ldots) .
$$

Let $\tau$ denote the map

$$
(x, y) \rightarrow x-y
$$

of $G \times G$ onto $G$, and put 


$$
W_{n}=\tau^{-1}\left(V_{n}\right)
$$

We shall prove that $\tau^{-1}(N)$ is locally negligible.

For any compact set $C \subset G$ consider the square $C \times C \subset G \times G$. Clearly, since $C \times C$ is compact, $V_{n}$ is open and $\tau$ is continuous, we have that $\xi_{C \times C}$ and $\xi_{W_{n}}$ are measurable and

$$
\xi_{W_{n}} \xi_{C \times C} \in L^{I}(G \times G)
$$

By Fubini's theorem (Hewitt and Ross [1], (13.8))

$$
\int_{G \times G} \xi_{W_{n}} \xi_{C \times C} d \lambda \times \lambda=\int_{G}\left\{\int_{G} \xi_{W_{n}}(x, y) \xi_{C \times C}(x, y) d \lambda(x)\right\} d \lambda(y)
$$

and, since

$$
W_{n}=\left\{(x, y): x \in y+V_{n}\right\},
$$

we have that

$$
\begin{aligned}
\int_{G \times G} \xi_{W_{n}} \xi_{C \times C} d \lambda \times \lambda & \leq \int_{G}\left\{\int_{G} \xi_{W_{n}}(x, y) d \lambda(x)\right\}_{C}(y) d \lambda(y) \\
& =\lambda\left(V_{n}\right) \lambda(C) \\
& \leq n^{-1} \lambda(C)
\end{aligned}
$$

that is,

$$
\lim _{n \rightarrow \infty} \lambda \times \lambda\left(W_{n} \cap(C \times C)\right)=0
$$

As the compact set $C$ was chosen arbitrarily it follows that $\tau^{-1}(N)$ $\left(\subset \tau^{-1}\left(V_{n}\right)=W_{n}\right)$ is locally negligible.

Now, by Hewitt and Ross [1], (11.41), there exists a Borel measurable function $f_{0}$ on $G$ such that $f=f_{0}$ almost everywhere. Clearly

$$
F_{0}:(x, y) \rightarrow f_{0}(x-y)
$$

is Borel measurable, hence measurable, and by what we proved in the previous paragraph

$$
F=F_{0} \quad \text { l.a.e. . }
$$


The measurability of $F$ follows from Hewitt and Ross [1], (11.30).

Suppose now that we just have that $f$ is measurable (and, without loss of generality, bounded). For any compact set $K \subset G \times G$ choose a compact set $C \subset G$ such that

$$
K \subset C \times C
$$

Then, using the fact that

$$
C \times C \subset \tau^{-1}(C-C),
$$

we see that for any non-negative $r \in R$,

$$
\begin{aligned}
\left\{(x, y):\left|F \xi_{k}(x, y)\right|>r\right\} & =\{(x, y):|F(x, y)|>r\} \cap K \cap \tau^{-1}(C-C) \\
& =\left\{(x, y):\left|f(x, y) \xi_{C-C}(x-y)\right|>r\right\} \cap K .
\end{aligned}
$$

Since $f \xi_{C-C} \in L^{l}(G)$ and $K$ is measurable it follows (using what was proved in the first part of the proof) that $F \xi_{K}$ is $\lambda \times \lambda$-measurable for every compact set $K \subset G \times G$ and, by Hewitt and Ross [1], (11.42) (iii), $F$ must be $\lambda \times \lambda$-measurable.

$$
\text { // }
$$

We now have (but see Gaudry [1], Definition 2.16 for the definition of $\hat{f} \hat{g})$ :

A.1.2 THEOREM. Let $f \in L^{1}(G)$ and $g \in L^{p}(G)(p \in(1, \infty])$. Then (a) $(f * g)^{\wedge}=\hat{f} \hat{g}$, and

(b) $\left[(f * g)^{\wedge}\right] \subset \operatorname{supp}(\hat{f}) \cap[\hat{g}]$.

Proof. Let $h \in D(\Gamma)$. Then

$$
\begin{aligned}
(f * g)^{\wedge}(h) & =(f * g)\left(\hat{h}_{v}\right) \\
& =\int_{G} f * g(x) \hat{h}_{v}(x) d x \\
& =\int_{G}\left\{\int_{G} f(x-y) g(y) d y\right\} \hat{h}_{v}(x) d x .
\end{aligned}
$$

Now, since $g \in L^{P}(G)$ and $\hat{h}_{v} \in L^{l}(G)$, there must exist sets $A_{n}, B_{n}$ $(n \in\{1,2, \ldots\})$ of finite measure such that $g, \hat{h}_{v}$ vanish off $\bigcup_{n=1}^{\infty} A_{n}$,

$\infty$

$\cup B_{n}$ respectively. Hence $n=1$ 


$$
\psi:(x, y) \rightarrow f(x-y) g(y) \hat{h}_{v}(x)
$$

vanishes off the $\lambda \times \lambda$ - $\sigma$-finite set $\bigcup_{m, n=1}^{\infty} A_{m} \times B_{n}$. Furthermore, using Lemma A.1.1 and Hewitt and Ross [1], (13.12), it is clear that $\psi$ is $\lambda \times \lambda$-measurable. Finally

$$
\begin{aligned}
\int_{G}\left\{\int_{G}\left|f(x-y)\|g(y)\| \hat{h}_{v}(x)\right| d y\right\} d x & =\int_{G}|f| *|g|(x)\left|\hat{h}_{v}(x)\right| d x \\
& \leq\||f| *|g|\|_{p}\left\|\hat{h}_{v}\right\|_{p^{\prime}} \\
& <\infty
\end{aligned}
$$

and we can apply Hewitt and Ross [1], (13.10) to obtain

$$
\begin{aligned}
(f * g)^{\wedge}(h) & =\int_{G}\left\{\int_{G} f(x-y) \hat{h}_{\vee}(x) d x\right\} g(y) d y \\
& =\int_{G} f * \hat{h}(-y) g(y) d y \\
& =g\left((f * \hat{h})_{v}\right\} \\
& =\hat{g}(\hat{f} h) \\
& =\hat{f} g(h) .
\end{aligned}
$$

Since $h \in D(\Gamma)$ was chosen arbitrarily

$$
(f * g)^{\wedge}=\hat{f g},
$$

proving (a).

To prove (b) we show that

$$
\operatorname{supp}(\hat{f})^{c} \cup[\hat{g}]^{c} \subset\left[(f * g)^{\wedge}\right]^{c}
$$

If $\hat{f}$ vanishes on $\Omega$ and $h \in D(\Gamma)$ with $\operatorname{supp}(h) \subset \Omega$, then $\hat{f} h=0$ and

$$
\begin{aligned}
(f * g)^{\wedge}(h) & =\hat{f} \hat{g}(h) \\
& =\hat{g}(\hat{f} h) \\
& =0 .
\end{aligned}
$$

If $\hat{g}$ vanishes on $\Omega$ then, since $\operatorname{supp}(\hat{f} h) \subset \Omega$ for all $h \in D(\Gamma)$ with $\operatorname{supp}(h) \subset \Omega$, we have 


$$
\begin{aligned}
(f * g)^{\wedge}(h) & =\hat{f} \hat{g}(h) \\
& =\hat{g}(\hat{f} h) \\
& =0 .
\end{aligned}
$$

In both cases $(f * g)^{\wedge}$ vanishes on $\Omega$, as required. //

\section{A.2 The equivalence of two definitions of the spectrum}

We show that the definition (see 0.2 .6 ) of the spectrum of an

$L^{p}$-function $f$ agrees with that of defining the spectrum of $f$ to be the support of its Fourier transform.

A.2.1 THEOREM. Let $f \in L^{p}(G) \quad(p \in(1, \infty])$. Then

$$
\Sigma(f)=[\hat{f}] \text {. }
$$

If $f \in L^{1}(G)$ then

$$
\Sigma(f)=\operatorname{supp}(\hat{f})
$$

Proof. Initially we show that if $f \in L^{\infty}(G)$ then

$$
\sum(f) \subset[\hat{f}] \text {. }
$$

Let $\gamma \in \Sigma(f)$ and suppose that there exists an open neighbourhood $\Omega$ of $\gamma$ such that $\hat{f}(h)=0$ for every $h \in D(\Gamma)$ with $\operatorname{supp}(h) \subset \Omega$. Choosing $h \in D(\Gamma)$ with $\operatorname{supp}(h) \subset \Omega$ and $h(\gamma) \neq 0$ we have that for any $x \in G$,

$$
\begin{aligned}
\hat{h} * f(x) & =f\left(\left(\hat{h}_{x}\right)_{v}\right) \\
& =\hat{f}\left(\left(\varepsilon_{x}\right)^{\wedge} h\right) \\
& =0,
\end{aligned}
$$

the last step using the fact that $\left(\varepsilon_{x}\right)^{\wedge} h \in D(\Gamma)$ and $\operatorname{supp}\left(\left(\varepsilon_{x}\right)^{\wedge} h\right) \subset \Omega$. Since $h(\gamma) \neq 0$ and $\gamma \in \Sigma(f)$ we have a contradiction of the definition of $\Sigma(f)$; thus $(A .2 . I)$ must hold.

Now if $p \in(I, \infty]$ and $f \in L^{p}(G)$ then for any $\phi \in C_{00}(G)$ it follows that $\phi * f \in L^{\infty}(G)$ and, using (A.2.1) and Theorem A.1.2, 


$$
\begin{aligned}
\Sigma(f) & =\underset{\phi \in \mathcal{C}_{00}(G)}{U} \Sigma(\phi * f) \\
& \subset \bigcup_{\phi \in C_{00}(G)}^{U}\left[(\phi * f)^{\wedge}\right] \\
& \subset[\hat{f}] .
\end{aligned}
$$

To show the reverse inclusion let $\gamma \in[\hat{f}]$ and choose $\phi \in C_{00}(G)$ such that $\hat{\phi}(\gamma) \neq 0$. If $\gamma k \sum(\phi * f)$ then we could find $g \in L^{I}(G)$ such that $\hat{g}(\gamma) \neq 0$ and

$$
g^{\sim} * \phi * f=0
$$

Choose $\Omega^{\prime}$ to be any relatively compact open neighbourhood of $\gamma$ such that $\overline{\hat{g}} \hat{\phi}$ is bounded away from zero on $\Omega^{\prime}$, and an open set $\Omega$ such that

$$
\gamma \in \Omega \subset \Omega^{-} \subset \Omega^{\prime} \text {. }
$$

Then, using the Wiener-Lévy theorem, we have the existence of $l \in L^{l}(G)$ such that $\overline{\hat{g}} \hat{\phi} \hat{Z}=1$ on $\Omega^{-}$. Thus for every $h \in D(\Gamma)$ with $\operatorname{supp}(h) \subset \Omega$

$$
\begin{aligned}
\hat{f}(h) & =f\left(\hat{h}_{v}\right) \\
& =\hat{h} * f(0) \\
& =g^{\sim} * \phi * Z * \hat{h} * f(0) \\
& =0,
\end{aligned}
$$

and we have a contradiction of the choice of $\gamma \in[\hat{f}]$. Hence

$$
\gamma \in \Sigma(\phi * f) \subset \Sigma(f) \text {, }
$$

that is,

$$
[\hat{f}] \subset \Sigma(f)
$$

which, combined with $(A .2 .2)$, proves the first part of the theorem.

To prove the theorem for $f \in L^{l}(G)$ we make use of Reiter [1], Remark I, p. 139 and elementary properties of the closure operator to obtain

$$
\begin{aligned}
\Sigma(f) & =\underset{\phi \in C_{00}(G)}{U} \sum(\phi * f) \\
& =\underset{\phi \in C_{00}(G)}{U} \operatorname{supp}\left\{(\phi * f)^{\wedge}\right\} \\
& =\operatorname{supp}(\hat{f}) .
\end{aligned}
$$




\section{A.3 Strict local inclusion results between spaces of Fourier transforms}

Throughout this section $G$ is assumed to be non-compact and Abelian. Let $p \in(1, \infty]$ and put

$$
L^{p}(G)^{\wedge}=\left\{\hat{f}: f \in L^{p}(G)\right\} .
$$

We shall be interested in some sort of inclusion (cf. Katznelson [1], chapter VI, 4.12) between $L^{p}(G)^{\wedge}$ and $L^{q}(G)^{\wedge}$, where $I<p<q \leq \infty$. It is easily shown that if $\Omega$ is a relatively compact open subset of $\Gamma$ then for any $f \in L^{p}(G)$ there exists $g \in L^{q}(G)$ such that the quasimeasure $\hat{f}-\hat{g}$ vanishes on $\Omega$. We abbreviate this by writing

$$
L^{p}(G)^{\wedge} \stackrel{\operatorname{loc}}{\subset} L^{q}(G)^{\wedge}
$$

It appears that the "local" inclusion in (A.3.I) is strict, and we shall give both existential and constructive proofs of this. An even better result (Theorem A.3.6) is obtained when $G$ is assumed to be second countable.

A.3.1 THEOREM. Let $1<q \leq \infty$ and suppose $h \in L^{I^{+}}(G)$ with $h \neq 0$. Then there exists $f \in L^{q^{+}}(G)$ such that $h * f \notin L^{p}(G)$ for any $p \in[1, q)$.

Proof. We shall consider two cases:

i) $q=\infty$.

Here we just take $f=1$, since for all $x \in G$

$$
I * h(x)=\int_{G} h d \lambda \neq 0,
$$

and the non-zero constant functions are not in $L^{p}(G)$ for any $p<\infty$. ii) $q<\infty$.

Fix $p \in[l, q)$ and assume that $h * f \in L^{p}(G)$ for all $f \in L^{q}(G)$. Consider the map $T$, defined on $L^{q}(G)$ by

$$
T: f \rightarrow h * f \text {. }
$$

By assumption $T$ maps $L^{q}(G)$ into $L^{p}(G)$. $T$ is obviously linear and, 
for any $a \in G$,

$$
\begin{aligned}
a^{(T(f))} & =a^{(h * f)} \\
& =h *{ }_{a}^{f} \\
& =T\left({ }_{a} f\right)
\end{aligned}
$$

that is, $T$ commutes with translations. We shall prove that $T$ is continuous.

Suppose $\left(f_{n}\right)$ is a sequence of functions in $L^{q}(G)$ such that

$$
\left\|f_{n}\right\|_{q} \rightarrow 0 \text { and }\left\|h * f_{n}-g\right\|_{p} \rightarrow 0
$$

for some $g \in L^{p}(G)$. Now $\left\|f_{n}\right\|_{q} \rightarrow 0$ implies that $\left\|h * f_{n}\right\|_{q} \rightarrow 0$ and hence we can find a subsequence $\left(f_{n_{s}}\right)$ such that

$$
h * f_{n_{s}} \rightarrow 0 \text { a.e. }
$$

But from (A.3.2) we can deduce the existence of a sub-subsequence $\left(f_{n_{s}}\right)$ such that

$$
h * f_{n_{s_{t}}}-g \rightarrow 0 \text { a.e. }
$$

Hence $g=0$ almost everywhere and we appeal to the Closed Graph theorem to deduce that $T$ is continuous.

It now follows from a generalisation of Hörmander's theorem (see Gaudry [1], Section 5, p. 475) that $T=0$. But clearly, since $h \neq 0$, we can find $f \in L^{q}(G)$ such that $h * f \neq 0$, and so our assumption leads to a contradiction. Hence there exists $f \in L^{q}(G)$ such that $h * f k L^{p}(G)$ and, since $h \geq 0, h *|f| k L^{P}(G)$.

Now let $\left(p_{n}\right), p_{n} \geq 1$, be any strictly increasing sequence of numbers converging to $q$. Choose a corresponding sequence $\left(f_{n}\right)$ of nonnegative functions in $L^{q}(G)$ such that for each $n \in\{1,2, \ldots\}$,

$$
h * f_{n} \vDash L^{p_{n}}(G) .
$$


We assert that

$$
f=\sum_{n=1}^{\infty} n^{-2}\left\|f_{n}\right\|_{q}^{-1} f_{n}
$$

satisfies the conditions of the theorem (for $q<\infty$ ); indeed, if there exists $p \in[1, q)$ such that

$$
h * f \in L^{p}(G)
$$

then, choosing $n_{0}$ such that $p_{n_{0}} \in[p, q$ ), we would have (recall that for each $\left.n, h * f_{n} \geq 0\right)$

$$
h * f_{n_{0}} \in L^{p} \cap L^{q}(G) \subset L^{p} n_{0}(G)
$$

a contradiction of $(\mathrm{A} .3 .3)$. //

A.3.2 THEOREM. Let $1<q \leq \infty$ and suppose $\Omega$ is a non-void open subset of $\Gamma$. Then there exists $f \in L^{q}(G)$ such that for any $p \in[1, q)$ and $g \in L^{p}(G), \hat{f}-\hat{g}$ does not vanish on $\Omega$.

Proof. Choose $\gamma \in \Omega$ and non-zero $h \in D(\Gamma)$ such that $\operatorname{supp}(h) \subset-\gamma+\Omega$ and $\hat{h} \geq 0$ (this is possible using Gaudry [1], Lemma 2.9). From Theorem A.3.I there exists $f_{0} \in t^{+}(G)$ such that $\hat{h} * f_{0} k L^{p}(G)$ for any $p \in[l, q)$. Now put $f=\gamma f_{0}$. It is clear that $f$ thus chosen satisfies the conditions of the theorem, for if there exists $p \in[1, q)$ and $g \in L^{P}(G)$ such that $\hat{f}-\hat{g}$ vanishes on $\Omega$ then, since $(\bar{\gamma} f-\bar{\gamma} g)^{\wedge}$ vanishes on $-\gamma+\Omega$, we would have

$$
x^{(\hat{h}) *(\bar{\gamma} f-\bar{\gamma} g)(0)=0}
$$

that is,

$$
\hat{h} *(\bar{\gamma} f)=\hat{h} *(\bar{\gamma} g)
$$

But $\hat{h} *(\bar{\gamma} g) \in L^{p}(G)$ and hence so is $\hat{h} * f_{0}$, a contradiction of our choice of $f_{0} \cdot \quad / /$

For completeness we state our desired result as: 
A.3.3 COROLLARY. Let $G$ be a non-compact locally compact Abelian group and suppose $1<p<q \leq \infty$. Then

$$
L^{p}(G) \stackrel{\perp q^{c}}{\ddagger} L^{q}(G) .
$$

We are also able to construct a function $f \in L^{q}(G)$ satisfying the conditions of Theorem A.3.2. We require a preliminary result.

A.3.4 THEOREM. Let $\Omega$ be a non-void open neighbourhood of zero in $\Gamma$. Then, for $q \in(1, \infty]$, we can find $f \in L^{q}(G)$ such that

(a) $f \geq 0$,

(b) $[\hat{f}] \subset \Omega$, and

(c) $f \notin L^{p}(G)$ for any $p \in[1, q)$.

Proof (by construction). Since, for $q=\infty, f=1$ trivially satisfies conditions $(a)-(c)$ we shall assume that $q<\infty$.

Choose $\nabla$ to be any relatively compact open symmetric neighbourhood of zero such that

$$
2 \nabla \subset \Omega
$$

Define $\phi$ on $\Gamma$ by

$$
\phi=\xi_{\nabla} * \xi_{\nabla}^{\sim},
$$

and put $u=\hat{\phi}$ (it is clear that $\phi \in A(\Gamma)$ ). Then $\phi \in C_{00}^{+}(\Gamma)$, $\operatorname{supp}(\phi) \subset \Omega$ and $u \in L^{I^{+}} \cap C_{0}(G)$.

Now we can find $\alpha>0$ and a non-void compact set $C \subset G$ for which $u \geq \alpha \xi_{C}$. Since $G$ is non-compact we can find a sequence $\left(x_{m}\right), x_{m} \in G$, such that for $m \neq n$

$$
\left(x_{m}+c\right) \cap\left(x_{n}+c\right)=\square
$$

and

$$
\left(x_{m}+C-c\right) \cap\left(x_{n}+C-C\right)=\square .
$$




$$
f_{1}=\sum_{m=1}^{\infty} \alpha_{m} \xi_{x_{m}}+C-C,
$$

where

$$
\alpha_{m}=n^{-2 / q_{2}-n / q}
$$

and

$$
S_{n}=\left[2^{n-1}, 2^{n}\right) \cap Z \quad(n=1,2, \ldots) .
$$

Then, using (A.3.5),

$$
\begin{aligned}
\left\|f_{1}\right\|_{q}^{q} & =\sum_{m=1}^{\infty} a_{m}^{q} \lambda\left(x_{m}+c-c\right) \\
& =\lambda(c-c) \sum_{n=1}^{\infty} n^{-2} 2^{-n} 2^{n-1} \\
& <\infty .
\end{aligned}
$$

Thus $f_{1} \in L^{q}(G)$ and hence so is

$$
f=f_{1} * u \text {. }
$$

Clearly $f \geq 0$ and $[\hat{f}] \subset \Omega$.

To show (c), choose $p \in[1, q)$ and consider

$$
\begin{aligned}
\int_{G} f^{p} d \lambda & =\int_{G}\left\{\int_{G} f_{I}(x-y) u(y) d y\right\}^{p} d x \\
& \geq \alpha^{p} \int_{G}\left\{\int_{C} f_{I}(x-y) d y\right\}^{p} d x \\
& =\alpha^{p} \int_{G}\left\{\sum_{m=1}^{\infty} \alpha_{m} \int_{C} \xi_{x_{m}}+C-C(x-y) d y\right\}^{p} d x \\
& =\alpha^{p} \int_{G}\left\{\sum_{m=1}^{\infty} \alpha_{m} \int_{C} \xi_{x-x_{m}+C-C}(y) d y\right\}^{p} d x \\
& \geq \alpha^{p} \int_{G}\left\{\sum_{m=1}^{\infty} \alpha_{m} \xi_{x_{m}+C} \lambda\left(C \cap\left(x-x_{m}+C-C\right)\right)\right\}^{p} d x
\end{aligned}
$$

Now if $x \in x_{m}+C$ then $C \subset x-x_{m}+C-C$ and we have, using (A.3.4), 


$$
\begin{aligned}
\int_{G} f^{p} d \lambda & \geq \alpha^{p} \sum_{m=1}^{\infty} \alpha_{m}^{p} \lambda(c)^{p} \int_{G} \xi_{x_{m}+c} d \lambda \\
& =\alpha^{p} \sum_{m=1}^{\infty} \alpha_{m}^{p} \lambda(c)^{p+1} \\
& =\lambda(C)^{p+1} \frac{\alpha^{p}}{2} \sum_{n=1}^{\infty} n^{-\frac{2 p}{q}} 2^{-n\left(\frac{p}{q}-1\right)} .
\end{aligned}
$$

Since $p<q$ the series diverges to infinity and thus $f k L^{p}(G)$.

A.3.5 COROLLARY. Let $I<q \leq \infty$ and suppose $\Omega$ is a non-void open subset of $\Gamma$. Then we can find $f \in L^{q}(G)$ such that for any $p \in[1, q)$ and $g \in L^{p}(G), \hat{f}-\hat{g}$ does not vanish on $\Omega$.

Proof (by construction). We can assume without loss of generality that $0 \in \Omega$. Let $\Omega^{\prime}$ be a relatively compact open set such that

$$
0 \in \Omega^{\prime} \subset \Omega^{\prime-} \subset \Omega \text {. }
$$

Using Theorem A.3.4 we can construct $f \in L^{q}(G)$ such that $f \geq 0$, $[\hat{f}] \subset \Omega^{\prime}$ and $f \equiv L^{p}(G)$ for any $p \in[1, q)$.

To show that $f$ so constructed satisfies the conditions of the corollary, assume to the contrary that there exists $p \in[1, q)$ and $g \in L^{P}(G)$ such that $\hat{f}-\hat{g}$ vanishes on $\Omega$. Then, choosing $k \in L^{I}(G)$ such that

$$
\xi_{\Omega^{\prime-}} \leq \hat{k} \leq \xi_{\Omega}
$$

we have (making use of Theorem A.1.2)

$$
\begin{aligned}
\hat{f}(h) & =\hat{k} \hat{f}(h) \\
& =\hat{f}(\hat{k} h) \\
& =\hat{g}(\hat{k} h) \\
& =\hat{k} \hat{g}(h) \\
& =\left[(k * g)^{\wedge}\right](h)
\end{aligned}
$$

Hence, since the Fourier transform is one-to-one on $L^{q}(G)$,

$$
f=k * g \in L^{p}(G),
$$


and we have a contradiction of Theorem A.3.4.

/1

Our final result in this appendix is not quite as general (with respect to the underlying group) as Theorem A.3.2 but, in return, gives the existence of a suitable function $f$ which is independent of the choice of the non-void open set $\Omega$.

A.3.6 THEOREM. Let $G$ be a second countable non-compact locally compact Abelian group. If $I<p<q \leq \infty$ then there exists $f \in L^{q}(G)$ such that, for any non-void open set $\Omega \subset \Gamma$, there is no $g \in L^{p}(G)$ for which $\hat{f}-\hat{g}$ vanishes on $\Omega$.

Proof. Since $G$ is second countable so is $\Gamma$ (see Hewitt and Ross [1], (24.14)\}. Suppose to the contrary that no such $f$ exists. We shall make use of Baire's category theorem to derive a contradiction.

For each positive integer $m, n$ define $T_{m}\left(\Omega_{n}\right\}=\left\{f \in L^{q}(G): \hat{f}-\hat{g}\right.$ vanishes on $\Omega_{n}$ for some $\left.g \in L^{p}(G),\|g\|_{p} \leq m\right\}$, where $\left\{\Omega_{n}: n=1,2, \ldots\right\}$ is a base for the topology of $\Gamma$ of non-void open sets. We shall prove that

$$
\bigcup_{m, n=1}^{\infty} T_{m}\left(\Omega_{n}\right)=L^{q}(G)
$$

and that for each $m, n \in\{1,2, \ldots\}$,

$$
T_{m}\left(\Omega_{n}\right) \text { is closed. }
$$

Suppose (A.3.6) does not hold. Then there exists $f \in L^{q}(G)$ such that for any $m, n$ and $g \in L^{p}(G)$ where $\|g\|_{p} \leq m, \hat{f}-\hat{g}$ does not vanish on $\Omega_{n}$. But $\left\{\Omega_{n}\right\}_{n=1}^{\infty}$ is a base for the topology of $\Gamma$, whence it follows that for any non-void open set $\Omega \subset \Gamma$ there is no $g \in L^{p}(G)$ for which $\hat{f}-\hat{g}$ vanishes on $\Omega$; a contradiction of our initial assumption. Hence (A.3.6) holds.

To prove $(A .3 .7)$ choose positive integers $m, n$ and let $\left(f_{S}\right)$ be a 
sequence of functions in $T_{m}\left(\Omega_{n}\right)$ converging in $L^{q}(G)$ to $f$, say. Now for each $s \in\{1,2, \ldots\}$ there exists $g_{s} \in L^{p}(G)$ such that $\left\|g_{s}\right\|_{p} \leq m$ and $\hat{f}_{s}-\hat{g}_{s}$ vanishes on $\Omega_{n}$. Using the theorems of Alaoglu and Smulian we can deduce the existence of $g \in L^{p}(G)$ and a subsequence $\left(g_{s_{t}}\right) \subset\left(g_{s}\right)$ such that for every $h \in L^{P^{\prime}}(G)$,

$$
\int_{G} g_{s_{t}} h d \lambda \rightarrow \int_{G} g h d \lambda
$$

Hence, given $\varepsilon>0$ and $h \in L^{p^{\prime}}(G)$ with $\|h\|_{p^{\prime}} \leq 1$, we can find $t_{0}$ such that

$$
\begin{aligned}
\left|\int_{G} g h d \lambda\right| & \leq\left|\int_{G} g_{s_{t_{0}}} h d \lambda\right|+\varepsilon \\
& \leq m\|h\|_{p^{\prime}}+\varepsilon \\
& \leq m+\varepsilon,
\end{aligned}
$$

and since $h \in L^{P^{\prime}}(G)$ with. $\|h\|_{p^{\prime}} \leq 1$ was chosen arbitrarily,

$$
\|g\|_{p} \leq m+\varepsilon \text {. }
$$

But $\varepsilon>0$ was also chosen arbitrarily, whence it follows that

$$
\|g\|_{p} \leq m
$$

Now let $\varepsilon>0$ and $h \in D(\Gamma)$ with $\operatorname{supp}(h) \subset \Omega_{n}$ be given. Choose $s_{t_{0}}$ such that

$$
\left|\int_{G} g \hat{h}_{v} d \lambda-\int_{G} g_{s_{t_{0}}} \hat{h}_{v} d \lambda\right|<\varepsilon / 2
$$

and

$$
\left|\int_{G} f \hat{h}_{v} d \lambda-\int_{G} f_{s_{t_{0}}} \hat{h}_{v} d \lambda\right|<\varepsilon / 2
$$

(note that $\hat{h} \in L^{p^{\prime}}(G)$ ). Then 


$$
\begin{aligned}
|(\hat{f}-\hat{g})(h)| & =\mid(f-g)\left(\hat{h}_{v}\right) \\
& \leq\left|\int_{G} f \hat{h}_{v} d \lambda-\int_{G} f_{s_{t_{0}}} \hat{h}_{v} d \lambda\right|+\left|\int_{G} f_{s_{t_{0}}} \hat{h}_{v} d \lambda-\int_{G} g_{s_{t_{0}}} \hat{h}_{v} d \lambda\right| \\
& \left.<\varepsilon / 2+\mid \hat{f}_{s_{t_{0}}}-\hat{g}_{s_{t_{0}}}\right)(h)+\varepsilon / 2 .
\end{aligned}
$$

But $\hat{f}_{s_{t_{0}}}-\hat{g}_{s_{t_{0}}}$ vanishes on $\Omega_{n}$ and so

$$
|(\hat{f}-\hat{g})(h)|<\varepsilon .
$$

Since $\varepsilon>0$ and $h \in \mathcal{D}(\Gamma)$ with $\operatorname{supp}(h) \subset \Omega_{n}$ were chosen arbitrarily we deduce that $\hat{f}-\hat{g}$ vanishes on $\Omega_{n}$, proving (A.3.7).

Now $L^{P}(G)$ is a complete metric space and thus we can apply Baire's category theorem which gives us the existence of $m_{0}, n_{0} \in Z^{+}$such that $T_{m_{0}}\left(\Omega_{n_{0}}\right)$ has non-void interior. This means we can find $\delta>0$ and $f_{0} \in T_{m_{0}}\left\{\Omega_{n_{0}}\right\}$ such that

$$
V=\left\{f \in L^{q}(G):\left\|f-f_{0}\right\|_{q}<\delta\right\} \subset T_{m_{0}}\left(\Omega_{n_{0}}\right) .
$$

Let $k \in L^{q}(G)$. Choose non-zero $\alpha \in C$ such that

$$
\|\alpha k\|_{q}<\delta / 2
$$

and $f_{1} \in L^{q}(G)$ such that

$$
\left\|f_{1}-f_{0}\right\|_{q}<\delta / 2
$$

Then $f_{1} \in V$ and hence there exists $g_{1} \in L^{p}(G)$ such that $\hat{f}_{1}-\hat{g}_{1}$ vanishes on $\Omega_{n_{0}} \cdot$ Similarly, since

$$
\begin{aligned}
\left\|\alpha k+\left(f_{1}-f_{0}\right)\right\|_{q} & \leq\|\alpha k\|_{q}+\left\|f_{1}-f_{0}\right\|_{q} \\
& <\delta,
\end{aligned}
$$

we must have $\alpha k+f_{1} \in V$ and so, there exists $g_{2} \in L^{p}(G)$ such that 
$\left(\alpha k+f_{1}\right)^{\wedge}-\hat{g}_{2}$ vanishes on $\Omega_{n_{0}}$. Linearity of the Fourier transform entails that

$$
\left(\alpha k+f_{1}-g_{2}\right)^{\wedge}-\left(f_{1}-g_{1}\right)^{\wedge}=\left(\alpha k+g_{1}-g_{2}\right)^{\wedge}
$$

vanishes on $\Omega_{n_{0}}$ or, equivalently,

$$
\hat{k}-\alpha^{-1}\left(g_{2}-g_{1}\right)^{\wedge}
$$

vanishes on $\Omega_{n_{0}}$. But $\alpha^{-1}\left(g_{2}-g_{1}\right) \in L^{p}(G)$; since $k \in L^{q}(G)$ was chosen arbitrarily we have a contradiction of Theorem A.3.2.

Thus our initial assumption was false and the theorem is proved. 


\section{APPENDIX B}

\section{COMMENTS ON A THEOREM OF GODEMENT}

As promised in the introduction to Chapter 1 we shall give a statement of a restricted version of Theorem 2.6.1 of Rudin [1]; this has proved necessary since Professor Robert E. Edwards has pointed out that if Rudin's proof is still to apply then there are two flaws in the statement of the theorem.

\section{B.1.1 THEOREM. Suppose $T$ is a compact subset of $\Gamma$ and that} $\Omega \subset \Gamma$ is relatively compact and Borel measurable with $\theta(\Omega)>0$. Then there exists $k \in L^{l}(G)$ such that

$$
\xi_{\Upsilon} \leq \hat{k} \leq \xi_{\Upsilon+\Omega-\Omega}
$$

and

$$
\|k\|_{1} \leq\left(\frac{\theta(T-\Omega)}{\theta(\Omega)}\right)^{\frac{1}{2}} .
$$

This result first appeared in Godement [1] (Lemma II) where $\Omega$ is assumed to be open rather than Borel measurable. The proof given by Godement (and also by Rudin) requires that $T-\Omega$ should be $\theta$-measurable with finite measure. It appears that we can only guarantee this when $\Omega$ is relatively compact and Borel measurable (that $T-\Omega$ is Borel measurable (hence $\theta$-measurable) when $\Omega$ is Borel measurable follows readily from the first paragraph of the proof of Theorem 10.23 of Hewitt and Stromberg [1] and the fact that $T-\Omega$ is open (closed) whenever $\Omega$ is open (closed)). To show that it is not sufficient to assume merely that $\theta(\Omega)<\infty$ nor that $\Omega$ is $\theta$-measurable, consider the following examples:

(a) If $T$ is any compact set with strictly positive measure and $\Omega$ is open but not relatively compact then, by Henstock and Macbeath [1], Theorem 4, $\theta(T-\Omega)=\infty$ (even if $\theta(\Omega)<\infty)$. 
(b) Following Kneser [1], p. 88, 2) we take $\Xi \subset R$ to be any nonmeasurable set. Then $[-1,1] \times\{0\}$ is compact and $\{0\} \times(-\Xi)$ is negligible (hence measurable) but

$$
[-1,1] \times\{0\}-\{0\} \times(-\Xi)=[-1,1] \times \Xi
$$

is non-measurable. 


\section{APPENDIX C}

\section{ON CERTAIN CLOSED SUBSETS OF A LOCALLY COMPACT ABELIAN GROUP}

This appendix, which is essentially a rewritten version of Bloom [3], arises from an attempt to relate the sets $B_{\mathrm{T}}^{p}(G)$ to sets of the form $L_{\Delta}^{P}(G)$ for some $\Delta \subset \Gamma$; see Theorem 1.3.4. Assuming throughout that $G$ is a Hausdorff locally compact Abelian group we introduce certain closed subsets $\left(S_{p^{-}}, C_{p^{-s e t}}\right)$ of $\Gamma$ by generalising one of the definitions of an S-set. We then obtain some properties and examples of these sets, and finally we use a proof of Malliavin's theorem on the failure of spectral synthesis in $L^{l}(G)^{\wedge}$ to show that every non-discrete $\Gamma$ contains a closed set which is not an $S_{p}$-set for any $p \in[1,2)$.

\section{C.1 The definition and some properties of $S_{p}-$ and $c_{p}$-sets}

C.1.1 DEFINITION. Let $\Xi$ be a closed subset of $\Gamma$. We shall call $\Xi$ an $S_{p}$-set $\{p \in[1, \infty)\}$ if, given $\varepsilon>0$ and $f \in L^{I} \cap L^{p}(G)$ such that $\hat{f}$ vanishes on $\Xi$, there exists $g \in L^{1} \cap L^{p}(G)$ such that $\hat{g}$ vanishes on a neighbourhood of $\Xi$ and

$$
\|f-g\|_{p}<\varepsilon
$$

If such a $g$ can be found of the form

$$
g=h * f,
$$

where $h \in L^{l}(G)$ and $\hat{h}$ vanishes on a neighbourhood of $\Xi$, then $\Xi$ will be called a $C_{p}$-set. We also define $S_{\infty}-$ and $C_{\infty}$-sets as above, with $f, g$ in $L^{1} \cap C_{0}(G) \quad$ rather than $L^{1} \cap L^{\infty}(G)$ ). 
Since, by Hewitt and Ross [1], (33.12), $L^{I}(G)$ admits a bounded positive approximate identity $\left\{u_{\imath}\right\}_{\imath \in I}$ such that for each $\iota \in I$, $u_{\imath} \in L^{I} \cap C_{0}(G)$ and $\operatorname{supp}\left(\hat{u}_{\imath}\right)$ is compact, it follows (see Hewitt and Ross [1], (32.33) (b) and (32.48) (a)) that we can (and shall) assume in Definition C.I.I that $f, g, h \in L^{l} \cap C_{0}(G)$, where $\operatorname{supp}(\hat{f})$ is compact and both $\operatorname{supp}(\hat{g})$ and $\operatorname{supp}(\hat{h})$ are compact and disjoint from $\Xi(p \in[1, \infty])$.

Clearly every $C_{p}$-set is an $S_{p}$-set. For the case $p=1$ we just have the familiar $S$-set and $C$-set; see Rudin [1], 7.2 .5 (a) and 7.5.1 respectively. The following theorem gives a characterisation of $S_{p}{ }^{-}$and $C_{p}$-sets. This result is known for the case $p=1$ (see Reiter [1], Chapter 7, 1.2, and Chapter 7, 4.9).

C.1.2 THEOREM. Let $p \in[1, \infty)$ and suppose $\Xi$ is a closed subset of $\Gamma$. Then

(a) $\Xi$ is an $S_{p}$-set if and only if for all $l \in L_{\Xi}^{p^{\prime}}(G)$ and for all $f \in L^{I} \cap C_{0}(G)$ such that $\operatorname{supp}(\hat{f})$ is compact and $\hat{f}$ vanishes on $E$, we have

$$
\tau * f=0 \text {; }
$$

(b) $\Xi$ is a $C_{p}$-set if and only if for alz $f \in L^{I} \cap C_{0}(G)$ such that $\operatorname{supp}(\hat{f})$ is compact and $\hat{f}$ vonishes on $\Xi$, and for alz $z \in L^{P^{\prime}}(G)$ such that $z * f \in L_{\Xi}^{p^{\prime}}(G)$, we have

$$
\imath * f=0 \text {. }
$$

Proof. (a) Assume $\Xi$ is an $S_{p}$-set. Let $l \in L_{\Xi}^{p^{\prime}}(G)$, $f \in L^{I} \cap C_{0}(G)$ with $\operatorname{supp}(\hat{f})$ compact and $\hat{f}$ vanishing on $\Xi$, and $\varepsilon>0$ be given. There exists $g \in L^{I} \cap C_{0}(G)$ such that $\hat{g}$ vanishes on a 
neighbourhood of $\Xi$ and

$$
\|f-g\|_{p}<\varepsilon
$$

Then

$$
\tau * g=0
$$

and

$$
\begin{aligned}
\|z * f\|_{\infty} & =\|z *(f-g)\|_{\infty} \\
& \leq \varepsilon\|z\|_{p^{\prime}} .
\end{aligned}
$$

Since $\varepsilon>0$ was chosen arbitrarily

$$
\tau * f=0 \text {. }
$$

Conversely suppose $\Xi$ is not an $S_{p}$-set. Then there exists

$f \in L^{I} \cap C_{0}(G)$ such that $\operatorname{supp}(\hat{f})$ is compact, $\hat{f}$ vanishes on $\Xi$ and $f$ is not in $w$, the $L^{p}$-closure of $\left\{g \in L^{I} \cap C_{0}(G): \operatorname{supp}(\hat{g})\right.$ is compact and $\hat{g}$ vanishes

on a neighbourhood of $\Xi\}$.

By the Hahn-Banach theorem there exists a continuous linear functional $T$

on $L^{P}(G)$ such that $T(W)=\{0\}$ and $T(f) \neq 0$. From Hewitt and Ross [1], (12.18) there exists $Z \in L^{p^{\prime}}(G)$ such that for all $g \in W$,

$$
T(g)=\int_{G} g \tau_{v} d \lambda .
$$

Then

$$
\tau * g(0)=0
$$

for all $g \in W$, whence it follows that

$$
\Sigma(Z) \subset \Xi
$$

and so that $z \in L_{\Xi}^{P^{\prime}}(G)$. By assumption

$$
\text { Z*f=0, }
$$

and we have a contradiction of the fact that $T(f) \neq 0$. 
The proof of (b) is similar to that of $(a)$. //

It is easy to adapt the proof of Rudin [1], Theorem 7.5 .2 to give:

C.1.3 THEOREM. Let $p \in[1, \infty]$. Then:

(a) every one-point subset of $\Gamma$ is a $C_{p}$-set in $\Gamma$;

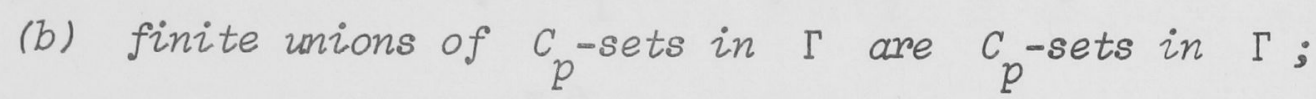

(c) if the boundary of a closed set $\Xi$ is a $C_{p}$-set, so is $\Xi$.

(d) if $\Xi$ is a closed subset of a closed subgroup $\Lambda$ of $\Gamma$, if $\partial_{\Lambda}(\Xi)$ is the boundary of $\Xi$ relative to $\Lambda$, and if $\partial_{\Lambda}(\Xi)$ is a $C_{p}$-set in $\Gamma$ then $\Xi$ is also a $C_{p}$-set in $\Gamma$ :

(e) each closed subgroup of $\Gamma$ is a $C_{p^{-s e t}}$ in $\Gamma$.

For $p \in[1,2)$ it is not known whether the notions of $C_{p}$-set and $S_{p}$-set are identical (it appears in Theorem C.2.I that every closed set is a $C_{p}$-set for $p \geq 2$ ). Furthermore we cannot say whether the union of two $S_{p}$-sets is itself an $S_{p}$-set. We can however obtain two partial results in this direction. Both these results (Theorems C.1.4 and C.1.5) are known for the case $p=1$ (see Reiter [1], Chapter 2, 7.5). Although their proofs are quite standard we include them here for the sake of completeness.

C.1.4 THEOREM. Suppose $\Xi_{1} \Xi_{1} \cup \Xi_{2}$, where $\Xi_{1}$ and $\Xi_{2}$ are disjoint closed subsets of $\Gamma$. Then, for $p \in[1, \infty), \Xi$ is an $S_{p}$-set if and only if both $\Xi_{1}$ and $\Xi_{2}$ are $S_{p}$-sets.

Proof. Assume $\Xi_{1}$ and $\Xi_{2}$ are $S_{p}$-sets. Let $l \in L_{\Xi}^{P^{\prime}}(G)$ and $f \in L^{I} \cap C_{0}(G)$ with $\operatorname{supp}(\hat{f})$ compact and $\hat{f}$ vanishing on $\Xi$ be given. For $n \in\{1,2\}$ choose disjoint open sets $V_{n}$ sich that

$$
\Xi_{n} \subset V_{n}
$$


and $k_{n} \in L^{l}(G)$ such that $\hat{k}_{n}=1$ on a neighbourhood of $\Xi_{n} n \operatorname{supp}(\hat{f})$ and

$$
\operatorname{supp}\left(\hat{k}_{n}\right) \subset V_{n}
$$

Then

$$
\begin{aligned}
\tau * f & =k_{1} * \tau * f+k_{2} * z * f \\
& =0
\end{aligned}
$$

the last step using Theorem C.I.2 and the assumption that $\Xi_{1}$ and $\Xi_{2}$ are $S_{p}$-sets. By Theorem C.l.2 again $\Xi$ is an $S_{p}$-set.

Conversely if $l \in L_{\Xi_{1}}^{p^{\prime}}(G)$ and $f \in L^{I} \cap C_{0}(G)$ is such that $\operatorname{supp}(\hat{f})$ is compact and $\hat{f}$ vanishes on $\Xi$ then $\left(k_{1} * f\right)^{\wedge}$ vanishes on $\Xi$ and $l \in L_{\Xi}^{p^{\prime}}(G)$. Hence, by Theorem C.l.2 and the fact that $k_{1} * l * f=l * f$,

$$
\begin{aligned}
z * f & =z * k_{1} * f \\
& =0 .
\end{aligned}
$$

By Theorem C.I.2 again $\Xi_{1}$ is an $S_{p}$-set.

Similarlyso is $\Xi_{2}$. //

C.1.5 THEOREM. Let $p \in[1, \infty)$ and suppose $\Xi_{1}$ is an $S_{p}$-set and $\Xi_{2}$ is a $c_{p}$-set. Then $\Xi=\Xi_{1} \cup \Xi_{2}$ is an $S_{p}$-set.

Proof. Let $l \in L_{\Xi}^{P^{\prime}}(G), f \in L^{I} \cap C_{0}(G)$ with $\operatorname{supp}(\hat{f})$ compact and $\hat{f}$ vanishing on $\Xi$ and $\varepsilon>0$ be given. Since $\Xi_{2}$ is a $C_{p}$-set there exists $h \in L^{l}(G)$ such that $\hat{h}$ has compact support disjoint from $\Xi_{2}$ and

$$
\|f-h * f\|_{p}<\varepsilon
$$

Now $l * h \in L_{\Xi_{1}}^{p^{\prime}}(G)$ and hence, by Theorem C.l.2 and the assumption that $\Xi_{1}$ is an $S_{p}$-set, we have

$$
\tau * h * f=0
$$


This, combined with (C.I.I) and the fact that $\varepsilon>0$ was chosen arbitrarily, gives

$$
\tau * f=0 \text {. }
$$

By Theorem C.I.2 again $\Xi$ is an $S_{p}$-set. //

The final result of this section gives us an inclusion result between the set of $C_{p}$-sets (respectively $S_{p}$-sets) and the set of $C_{q}$-sets (respectively $C_{q}^{\text {-sets }}$ for $I \leq p<q \leq \infty$.

C.1.6 THEOREM. Let $1 \leq p<q \leq \infty$. Then every $C_{p}$-set (respectively $S_{p}-$ set) is a $C_{q}^{- \text {set }}$ (respectively $S_{q}$-set).

Proof. Assume $\Xi$ is a $C_{p}$-set. Let $f \in L^{I} \cap C_{0}(G)$ with $\operatorname{supp}(\hat{f})$ compact and $\hat{f}$ vanishing on $\Xi$ and $\varepsilon>0$ be given. We can find $h \in L^{I} \cap C_{0}(G)$ such that

$$
\|f-h * f\|_{q}<\varepsilon / 2
$$

Since $\Xi$ is a $C_{p}$-set there exists $g \in L^{I}(G)$ such that $\hat{g}$ has compact support disjoint from $\Xi$ and

$$
\|h\|_{p}\|f-g * f\|_{p}<\varepsilon / 2,
$$

where $p^{-1}+r^{-1}-q^{-1}=1$ (with the usual convention for the cases $p=1$ and $q=\infty)$. Now (see Hewitt and Ross [1], (20.18))

$$
\begin{aligned}
\|f-h * g * f\|_{q} & \leq\|f-h * f\|_{q}+\|h\|_{r}\|f-g * f\|_{p} \\
& <\varepsilon .
\end{aligned}
$$

It remains only to note that $h * g \in L^{I} \cap C_{0}(G)$ and $(h * g)^{\wedge}$ has compact support disjoint from $\Xi$.

The proof that every $S_{p}$-set is an $S_{q}$-set is similar. // 


\section{C.2 Examples of $S_{p}-$ and $C_{p}$-sets}

C.2.1 THEOREM. For $p \in[2, \infty]$ every closed subset of $\Gamma$ is a $C_{p}$-set.

Proof. In view of Theorem C.I.6 we need only prove the theorem for $p=2$.

Let $\Xi$ be a closed subset of $\Gamma$ and suppose we are given $\varepsilon>0$ and $f \in L^{I} \cap C_{0}(G)$ with $\operatorname{supp}(\hat{f})$ compact, $\hat{f}$ vanishing on $\Xi$ and $\|f\|_{I} \leq 1$. Now

$$
\Omega=\{\gamma \in \Gamma: \hat{f}(\gamma) \neq 0\}
$$

is a relatively compact open set and hence there exists a compact set $\Upsilon \subset \Omega$ such that

$$
\theta(\Omega \backslash T)<\varepsilon^{2}
$$

Choose an open set $\nabla$ such that

$$
T \subset \nabla \subset \nabla^{-} \subset \Omega,
$$

and $k \in L^{l} \cap C_{0}(G)$ such that

$$
\xi_{\Upsilon} \leq \hat{k} \leq \xi_{\nabla}
$$

Then, using Plancherel's theorem,

$$
\begin{aligned}
\|f-k * f\|_{2} & \left.=\iint_{\nabla \backslash T}|1-\hat{k}(\gamma)|^{2}|\hat{f}(\gamma)|^{2} d \gamma\right\}^{\frac{1}{2}} \\
& <\theta(\nabla \backslash \Upsilon)^{\frac{1}{2}} \\
& <\varepsilon ;
\end{aligned}
$$

and clearly, $\hat{k}$ has compact support disjoint from $\Xi$.

C.2.2 DEFINITION. Suppose $\Omega$ is a relatively compact open subset of $\Gamma$ and $\beta>0$. We shall call $\Omega$ a $\beta$-symmetry set if there exist nets $\left\{T_{\ell}\right\}_{\iota \in_{I}}$ and $\left\{\nabla_{\ell}\right\}_{\ell \in I}$ such that each $\tau_{\ell}$ is compact, $\left\{\nabla_{\ell}\right\}_{\iota \in I}$ is a base of symmetric open neighbourhoods of zero in $\Gamma$, partially ordered by

$$
\nabla_{\iota} \prec \nabla_{x} \text { if and only if } \nabla_{\iota} \supset \nabla_{x} \text {, }
$$




$$
\begin{aligned}
& \left(\mathrm{T}_{\iota}+2 \nabla_{\imath}\right)^{-} \subset \Omega \text { for each } \iota \in I \text {, and } \\
& \lim _{l \in I} \frac{\theta\left(\Omega \backslash T_{l}\right)^{\beta}}{\theta\left(\nabla_{\imath}\right)^{\prime}}=0 .
\end{aligned}
$$

C.2.3 THEOREM. Suppose we are given $\beta>0$ and a closed subset $\Xi$ of $\Gamma$ with the property that for any relatively compact set $T \subset \Xi^{c}$ there exists a $\beta$-symmetry set $\Omega$ such that

$$
T \subset \Omega \subset \Xi^{c}
$$

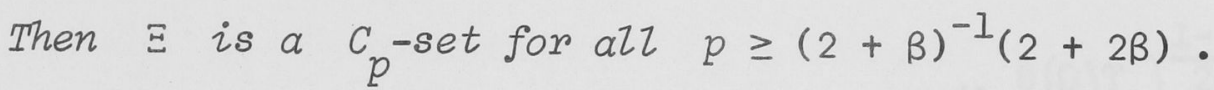

Proof. Let $p=(2+\beta)^{-1}(2+2 \beta)$. Suppose we are given $\varepsilon>0$ and $f \in L^{I} \cap C_{0}(G)$, where $\operatorname{supp}(\hat{f})$ is compact, $\hat{f}$ vanishes on $\Xi$ and $\|f\|_{1} \leq 1$. Now

$$
T=\{\gamma \in \Gamma: \hat{f}(\gamma) \neq 0\}
$$

is a relatively compact open subset of $\Xi^{c}$ and hence, by assumption, there exists a relatively compact open set $\Omega$ such that

$$
T \subset \Omega \subset \Xi^{c}
$$

and nets $\left\{\mathrm{T}_{\imath}\right\}_{\imath \epsilon_{I}}$ and $\left\{\nabla_{\imath}\right\}_{\imath \in I}$ satisfying the conditions of Definition C. 2.2 .

Choose $\imath \in I$ such that $T_{\imath}$ is non-void and

$$
\left(\frac{\theta\left(\Omega \backslash \tau_{\imath}\right)^{\beta}}{\theta\left(\nabla_{\imath}\right)}\right)^{\alpha / 2}<2^{-\alpha} \theta(\Omega)^{-\alpha / 2} \varepsilon,
$$

where $\alpha=(1+\beta)^{-1} \cdot$ Define

$$
k_{\imath}=\theta\left(\nabla_{\imath}\right)^{-1} g_{\imath} h_{\imath}
$$

where $g_{\imath}, h_{\imath}$ in $L^{2}(G)$ are such that $\hat{g}_{\imath}=\xi_{\nabla_{l}}$ and $\hat{h}_{\imath}=\xi_{q_{l}}+\nabla_{\iota}$. Then (see Theorem B.I.I) $k_{\ell} \in L^{I} \cap C_{0}(G), \quad \xi_{T_{\imath}} \leq \hat{k}_{\imath} \leq \xi_{T_{\imath}}+2 \nabla_{\imath}$ and 


$$
\left\|k_{\tau}\right\|_{I} \leq\left(\frac{\theta\left(r_{\tau}+\nabla_{\tau}\right)}{\theta\left(\nabla_{\tau}\right)}\right)^{\frac{7}{2}} .
$$

It follows from Hölder's inequality that

$$
\begin{aligned}
& \left\|f-k_{i} * f\right\|_{p} \leq\left\|f-k_{L} * f\right\|_{1}^{\alpha}\left\|f-k_{L} * f\right\|_{2}^{1-\alpha} \\
& \leq\|f\|_{I}^{\alpha}\left(1+\left(\frac{\theta\left(T_{\imath}+\nabla_{\tau}\right)}{\theta\left(\nabla_{\tau}\right)}\right)^{\frac{1}{2}}\right)^{\alpha} \theta\left(\Omega \backslash T_{\tau}\right)^{\frac{1-\alpha}{2}} \\
& \leq 2^{\alpha} \theta\left(\tau_{\imath}+\nabla_{\imath}\right)^{\alpha / 2} \frac{\theta\left(\Omega \backslash \tau_{\imath}\right)^{\frac{1-\alpha}{2}}}{\theta\left(\nabla_{\imath}\right)^{\alpha / 2}} \\
& <\varepsilon
\end{aligned}
$$

(recall that $\alpha=(1+\beta)^{-1}$ and $\left.p=(2+\beta)^{-1}(2+2 \beta)=2\left(1+\alpha^{\alpha-1}\right)^{-1}\right)$. Noting that $\hat{k}_{i}$ has compact support disjoint from $\Xi$ we see that $\Xi$ is a $C_{p}$-set, and the conclusion follows from Theorem C.1.6. //

We have two corollaries when $G$ is a Euclidean space.

C.2.4 COROLLARY. Let $m \geq 1$ and suppose $\Xi \subset R^{m}$ is an open set with the property that for any relatively compact set $\mathrm{T} \subset \mathrm{R}^{m}$ there exists a number $\kappa_{m}\left(=\kappa_{m}(T)\right)$ such that

$$
\theta\left((\partial(\Xi) \cap T)+\nabla_{n}\right) \leq \kappa_{m} n^{-1}
$$

for all $n \in\{1,2, \ldots\}$, where $\partial(\Xi)$ denotes the boundary of $\Xi$ and

$$
\nabla_{n}=\left\{x \in \mathbb{R}^{m}:\|x\|<n^{-1}\right\} .
$$

Then $\Xi, \Xi^{C}$ and $\partial(E)$ are $C_{p^{-s e t s}}$ for all $p>(2+m)^{-1}(2+2 m)$.

Proof. By Theorem C.1.3 (c) we need consider only $\partial(\Xi)$.

Let $T$ be any relatively compact open subset of $\partial(\Xi)^{c}$. We shall show that for any $\varepsilon>0$ there exists a $(m+\varepsilon)$-symmetry set $\Omega$ such that 
Since $T$ is relatively compact in $R^{m}$ there exists an integer $n_{0}>0$ such that

$$
\Upsilon \subset \Delta_{n_{0}}=\left\{x \in R^{m}:\|x\|<n_{0}\right\}
$$

For each $n \in\{1,2, \ldots\}$ define

$$
T_{n}=\left(\partial(\Xi)+\nabla_{n}\right)^{c} n\left(\Delta_{n_{0}} \Delta_{n_{0}-n^{-1}}^{-}\right)^{c} \cap \Delta_{n_{0}}
$$

Clearly $T_{n}$ is compact and

$$
\left(T_{n}+2 \nabla_{3 n}\right)^{-} \subset \Delta_{n_{0}} \cap \partial(\Xi)^{C}
$$

Putting

$$
\Omega=\Delta_{n_{0}} \cap \partial(\Xi)^{c},
$$

we have

$$
\begin{aligned}
\Omega \backslash T_{n} & =\Omega \cap \mathrm{T}_{n}^{c} \\
& =\left(\Omega \cap\left(\partial(\Xi)+\nabla_{n}\right)\right) \cup\left(\Omega \cap\left(\Delta_{n_{0}} \backslash \Delta_{n_{0}-n^{-1}}^{-}\right)\right) \\
& =\left\{\Delta_{n_{0}} \cap \partial(\Xi)^{c} \cap\left(\partial(\Xi)+\nabla_{n}\right)\right\} \cup\left(\Delta_{n_{0}} \cap \partial(\Xi)^{c} \cap\left(\Delta_{n_{0}} \backslash \Delta_{n_{0}-n^{-1}}^{-}\right)\right\} \\
& \subset\left(\Delta_{n_{0}} \cap\left(\partial(\Xi)+\nabla_{n}\right)\right) \cup\left(\Delta_{n_{0}} \backslash \Delta_{n_{0}-n^{-1}}\right) \\
& \subset\left(\left(\left(\Delta_{n_{0}}+\nabla_{I}\right) \cap \partial(\Xi)\right)+\nabla_{n}\right) \cup\left(\Delta_{n_{0}} \backslash \Delta_{n_{0}-n^{-1}}^{-}\right) .
\end{aligned}
$$

Hence, since $\Delta_{n_{0}}+\nabla_{1}$ is relatively compact,

$$
\theta\left(\Omega \backslash T_{n}\right) \leq \kappa_{m}\left(\Delta_{n_{0}}+\nabla_{1}\right) n^{-1}+O\left(n^{-1}\right)
$$

Using the fact that

$$
\theta\left(\nabla_{3 n}\right)=k_{m}^{\prime} 3^{-m} n^{-m}
$$




$$
\lim _{n \rightarrow \infty} \frac{\theta\left(\Omega \backslash T_{n}\right)^{m+\varepsilon}}{\theta\left(\nabla_{3 n}\right)}=0,
$$

and so $\Xi$ is a $(m+\varepsilon)$-symmetry set for all $\varepsilon>0$.

Thus $\partial(\Xi)$ satisfies the conditions of Theorem C.2.3 with $\beta=m+\varepsilon$, and hence is a $C_{p}$-set for all $p>(2+m)^{-1}(2+2 m)$. $1 /$

C.2.5 COROLLARY. Let $m \geq 1$ and put

$$
\Xi=\left\{x \in R^{m}:\|x\|=1\right\} \text {. }
$$

Then $E$ is a $C_{p}$-set for all $p>(2+m)^{-1}(2+2 m)$.

Proof. Let $\nabla$ be any relatively compact set in $\mathrm{R}^{m}$. Then

$$
\begin{aligned}
\theta\left((\Xi \cap \nabla)+\nabla_{n}\right) & \leq \theta\left(\Xi+\nabla_{n}\right) \\
& =\kappa_{m}^{\prime}\left(\left(1+n^{-1}\right)^{m}-\left(1-n^{-1}\right)^{m}\right) \\
& =O\left(n^{-1}\right),
\end{aligned}
$$

where $K_{m}^{\prime}$ is a constant. Now apply Corollary C.2.4. //

C.2.6 REMARK. For $m \geq 3$, Corollary $C .2 .5$ gives an example of a $C_{p}$-set $\left((2+m)^{-1}(2+2 m)<p<2\right)$ which is not an S-set; $c f$. Rudin [1], 7.3.2.

\section{C.3 The failure of certain closed sets to be $S_{p}$-sets}

In this section we use a proof along the lines of that of Malliavin's theorem (Rudin $[1], 7.6 .1$ ) to show that every non-discrete $\Gamma$ contains a closed set which is not an $S_{p}$-set for any $p \in[1,2)$. As in the proof of Rudin [1], Theorem 7.6.1, we first consider the cases:

(a) $\Gamma$ is an infinite compact group; and

(b) $\quad \Gamma=R$.

C.3.1 THEOREM. Let $G$ be on infinite discrete group. Then there exists a closed set $\Xi \subset \Gamma$ which is not an $S_{p}$-set for any $p \in[1,2)$. 
Proof. Consider the function $\phi_{1}$ on $G$ defined, as in Rudin [1], Theorem $7 \cdot 8 \cdot 5$, by

$$
\phi_{1}: x \rightarrow\left(D^{1} m_{x}\right)(\zeta)
$$

It is easily proved from Rudin [1], 7.6.4 and Theorem 7.8 .6 that $f_{0} \in L^{l}(G)$ and $\phi_{1}$ (as above) can be chosen so that $f_{0}$ and $\zeta$ satisfy the hypotheses of Rudin [1], 7.6.3 (Theorem) (with $f=f_{0}$ and $\xi=\zeta$ ) and

$$
\phi_{1} \in L^{q}(G)
$$

for all $q>2$. Having thus chosen $f_{0}$ and $\phi_{1}$ we shall prove that the closed set

$$
\Xi=\left\{\gamma \in \Gamma: \hat{f}_{0}(\gamma)=\zeta\right\}
$$

is not an $S_{p}$-set for any $p \in[1,2)$.

Let $p \in[1,2)$ and put

$$
I=\left\{f \in L^{I}(G): \hat{f}(\Xi)=\{0\}\right\},
$$

$I_{1}=\left\{f \in L^{I}(G): f\right.$ is in the closed ideal of $L^{I}(G)$ generated by $\left.f_{0}-\zeta\right\}$, $I_{2}=\left\{f \in L^{I}(G): f\right.$ is in the closed ideal of $L^{I}(G)$ generated by $\left.\left(f_{0}-\zeta\right)^{* 2}\right\}$,

and

$$
J=\left\{f \in L^{I}(G): \hat{f} \text { vanishes on a neighbourhood of } \Xi\right\}^{-} \text {. }
$$

Clearly

$$
\Xi=Z(I)=Z\left(I_{I}\right)=Z\left(I_{2}\right)=Z(J)
$$

(where $Z(I)$ denotes the zero set of the ideal. $I$; see Rudin [1], 7.1.3). Since $I$ and $J$ are respectively the largest and smallest closed ideals in $L^{l}(G)$ having $\Xi$ as their zero set, we have

$$
J \subset I_{2} \subset I_{1} \subset I
$$


As $\phi_{I} \in L^{P^{\prime}}(G)$ we can define a continuous linear functional $T$ on $\left(L^{I}(G),\|\cdot\|_{p}\right)$ by

$$
T(g)=\sum_{x \in G} g(-x) \phi_{1}(x)
$$

(recall that $G$ is discrete and hence $L^{l}(G) \subset L^{p}(G)$ ). By Rudin [l], 7.6.3, $T$ annihilates $I_{2}$ but not $I_{1}$.

Now suppose that $\Xi$ is an $S_{p}$-set and let $h \in L^{l} \cap C_{0}(G)=L^{l}(G)$ with $\hat{h}$ vanishing on $\Xi$. Then, given $\varepsilon>0$, there exists $h^{\prime} \in J$ such that

$$
\left\|h-h^{\prime}\right\|_{p}<\varepsilon
$$

and hence, since $T\left(h^{\prime}\right)=0$,

$$
\begin{aligned}
|T(h)| & =\left|T\left(h-h^{\prime}\right)\right| \\
& \leq \varepsilon\left\|\phi_{l}\right\|_{p^{\prime}} .
\end{aligned}
$$

Since $\varepsilon>0$ was chosen arbitrarily

$$
T(h)=0
$$

and thus $T$ annihilates $I$, a contradiction of the fact that $T$ does not annihilate $I_{I} \subset I$. It follows that $\Xi$ is not an $S_{p}$-set for any $p \in[1,2)$

We shall now examine the case when $\Gamma$ contains an infinite compact open subgroup. We require two lemmas for arbitrary Hausdorff locally compact Abelian groups.

C.3.2 LEMMA. Let $G$ be a Hausdorff locally compact Abelion group and suppose $H$ is a closed subgroup of $G$. Then a continuous integrable function $f$ on $G$ is constant on cosets of $H$ if and only if

$$
\operatorname{supp}(\hat{f}) \subset A(\Gamma, H) \text {. }
$$

Proof. Suppose that for all $x \in G$ and $h \in H$,

$$
f(x+h)=f(x) \text {. }
$$


Choose any $\gamma k A(\Gamma, H)$ and let $h \in H$ be such that $\gamma(h) \neq 1$. Then

$$
\hat{f}(\gamma)=\gamma(h) \hat{f}(\gamma) \text {, }
$$

and hence $\hat{f}(\gamma)=0$. Since $A(\Gamma, H)$ is closed

$$
\operatorname{supp}(\hat{f}) \subset A(\Gamma, H)
$$

Conversely, for $\gamma \in A(\Gamma, H)$ and $h \in H$,

$$
\hat{f}(\gamma)=\left(h_{h} f\right)^{\wedge}(\gamma)
$$

and since (using the assumption) the equality clearly holds for $\gamma k A(\Gamma, H)$ we see that

$$
h^{f}=f \quad a \cdot e .
$$

As $f$ is continuous the result follows. //

C.3.3 LEMMA. Let $G$ be a Hausdorff Zocally compact Abelian group and suppose $\Lambda$ is an open subgroup of $\Gamma$. If $\Xi$ is a closed subset of $\Lambda$ which is not an $S_{p}$-set in $\Lambda$ then $\Xi$ is not an $S_{p}$-set in $\Gamma$.

Proof. Put $H=A(G, \Lambda)$. By Hewitt and Ross [1], (23.24) (e), $H$ is compact. Furthermore, in view of Theorem C.2.1, we can assume that $p<\infty$.

Suppose, to the contrary, that $\Xi$ is an $S_{p}$-set in $\Gamma$. Given $\varepsilon>0$ and $\dot{f} \in L^{I} \cap C_{0}(G / H)$ such that $\operatorname{supp}(\hat{f})$ is compact and $\hat{\dot{f}}$ vanishes on $\Xi$, put

$$
f=\dot{f} \circ \pi_{H},
$$

where $\pi_{H}$ denotes the natural homomorphism of $G$ onto $G / H$. Denoting the Haar measures on $H, G / H$ by $\lambda_{H}, \lambda_{G / H}$ respectively (normalised as in Reiter [1], Chapter 3, 3.3 (i) with $\lambda_{H}(H)=1$ ) we have, by Reiter [1], Chapter 3, 4.5,

$$
\begin{aligned}
\|f\|_{p}^{p} & =\int_{G / H}\left\{\int_{H}|f(x+y)|^{p} d \lambda_{H}(y)\right\} d \lambda_{G / H}(\dot{x}) \\
& =\int_{G / H}\left\{\int_{H}\left|\dot{f} \circ \pi_{H}(x+y)\right|^{p} d \lambda_{H}(y)\right\} d \lambda_{G / H}(\dot{x}) \\
& =\int_{G / H}|\dot{f}(\dot{x})|^{p} d \lambda_{G / H}(\dot{x}),
\end{aligned}
$$


that is,

$$
\|f\|_{p}=\|\dot{f}\|_{p}
$$

It is easily seen that

$$
\dot{f}(\dot{x})=\int_{H} f(x+y) d \lambda_{H}(y)
$$

and, by Reiter [1], Chapter 4, 4.3 ((C.3.1) shows that $f \in L^{l}(G)$ ),

$$
\hat{f}(\gamma)=\hat{f}(\gamma)
$$

for all $\gamma \in \Lambda$. Furthermore, since $f$ is constant on cosets of $H$, Lemma C.3.2 shows that

$$
\operatorname{supp}(\hat{f}) \subset A(\Gamma, H)=\Lambda
$$

As $\operatorname{supp}(\hat{\dot{f}})$ is assumed to be compact it follows from (c.3.2) that $\operatorname{supp}(\hat{f})$ is compact and hence (note that $f$ is continuous) we see that $f \in C_{0}(G)$. Now $\hat{f}$ vanishes on $\Xi u \Omega^{c}$ and, since by Theorem C.1.4 (recall that $\Lambda^{C}$ is open and closed) $\Xi \cup \Lambda^{C}$ is an $S_{p}$-set, there exists $g \in L^{l} \cap C_{0}(G)$ such that $\hat{g}$ has compact support disjoint from $\Xi \cup \Lambda^{c}$ and

$$
\|f-g\|_{p}<\varepsilon \text {. }
$$

By Lemma C.3.2 again $g$ is constant on cosets of $H$ and we have the existence of $\dot{g} \in L^{l} \cap C_{0}(G / H)$ such that

$$
g=\dot{g} \circ \pi_{H}
$$

$\left(\dot{g} \in C_{0}(G / H)\right.$ since, by Reiter [1], Chapter 3, 1.8 (vii), $\dot{g}$ is continuous and by $(C .3 .2), \hat{\dot{g}}$ has compact support $)$. From (C.3.1)

$$
\|\dot{f}-\dot{g}\|_{p}<\varepsilon,
$$

and (C.3.2) shows that $\hat{\dot{g}}$ vanishes on a neighbourhood of $\Xi$. Hence $\Xi$ is shown to be an $S_{p}$-set in $\Lambda$, contrary to assumption. //

C.3.4 COROLLARY. Let $G$ be a Hausdorff locally compact Abelian group, $\Gamma$ its character group. If $\Gamma$ contains an infinite compact open subgroup then there exists a closed subset of $\Gamma$ which is not an $S_{p}$-set 
for any $p \in[1,2)$.

Proof. Combine Theorem C.3.I and Lemma C.3.3. //

Before considering the case $\Gamma=R$ we need to extend the result in Rudin [1], Theorem 2.7.6.

C.3.5 THEOREM. Suppose $f \in \ell^{l}(Z), \delta \in(0, \pi)$ and $\hat{f}(\exp (i x))=0$ for $x \in[\pi-\delta, \pi+\delta]$. Let $u$ be defined on $\mathrm{R}$ by

$$
u(x)= \begin{cases}\hat{f}(\exp (i x)) & (|x| \leq \pi) \\ 0 & (|x|>\pi) .\end{cases}
$$

Then $u=\hat{g}$ for some $g \in L^{l}(R)$. Moreover, given $p \in[1, \infty]$, there exists a positive number ${ }_{K_{p}}\left(=k_{p}(\delta)\right)$ such that

$$
\|f\|_{p} \leq k_{p}\|g\|_{p}
$$

Proof. The first part of Theorem C.3.5 is proved in Rudin [1], 2.7.6. Let $p \in[l, \infty]$. Consider the linear operator $T$ from $L^{l} \cap L^{\infty}(R)$ to $\ell^{\perp}(Z)$, defined by

$$
\{T(k)\}(n)=k * \hat{h}(n) \text {, }
$$

where $n \in Z$, and $h \in L^{l}(R)$ is defined as in Rudin [1], 2.7.6. The argument at the end of the proof of Rudin $[1], 2.7 .6$ shows that there is a constant $k_{1}=k_{1}(\delta)$ such that

$$
\|T(k)\|_{1} \leq k_{1}\|k\|_{1}
$$

It is clear from (C.3.3) that

$$
\|T(k)\|_{\infty} \leq k_{2}\|k\|_{\infty}
$$

where $k_{2}=\|\hat{h}\|_{1}$. By the Riesz-Thorin convexity theorem $T$ is continuous as

$$
\left\{L^{I} \cap L^{\infty}(R),\|\cdot\|_{p_{\alpha}}\right\} \stackrel{T}{\rightarrow}\left(\ell^{I}(\mathrm{z}),\|\cdot\|_{p_{\alpha}}\right\}
$$

(recall that $\left.\ell^{1}(Z) \subset \ell^{\infty}(Z)\right)$, where $\alpha \in(0,1), p_{\alpha}=(1-\alpha)^{-1}$ and 


$$
\|T\|_{(\alpha)} \leq \kappa_{1}^{1-\alpha_{K_{2}}}
$$

In particular, choosing $\alpha \in\left[0,1\right.$ ) such that $p_{\alpha}=p$ (and $\alpha=1$ if $p=\infty$ ) and noting that $g \in L^{1} \cap L^{\infty}(R)$ and (see Rudin $[1], 2.7 .6,(5)$ )

$$
f(n)=g * \hat{h}(n)
$$

for all $n \in Z$, we have

$$
\|f\|_{p} \leq k_{1}^{1-\alpha_{K_{2}}}\|g\|_{p}
$$

as required. $\quad / /$

C.3.6 THEOREM. The real line $R$ contains a closed set which is not an $S_{p}$-set for any $p \in[1,2)$.

Proof. It appears from Theorem C.3.I that there exists a closed set $\Xi_{I} \subset T$ which is not an $S_{p}$-set for any $p \in[1,2)$. By translation if necessary we can assume that $-1 k \Xi_{1}$ and $\Xi_{1} \cap \Xi_{2}=\square$ for some closed arc $\Xi_{2}$ containing -1 . Put

$$
\begin{aligned}
& T_{1}=\left\{x \in(-\pi, \pi): \exp (i x) \in \Xi_{1}\right\}, \\
& T_{2}=\left\{x \in(-\pi, \pi): \exp (i x) \in \Xi_{2}\right\} \cup[\pi, \infty) \cup(-\infty,-\pi], \\
& \Xi=\Xi_{1} \cup \Xi_{2},
\end{aligned}
$$

and

$$
T=T_{1} \cup T_{2}
$$

Let $p \in[1,2)$ and suppose $T_{1}$ is an $S_{p}$-set. By Theorem C.1.5, $T$ is an $S_{p}$-set $\left(\mathrm{T}_{2}\right.$ is a $C_{p}$-set since $\partial \mathrm{T}_{2}$ is finite; see Theorem $\left.\mathrm{C} .1 .3\right)$. Given $f \in \ell^{I}(Z)$ with $\hat{f}(\Xi)=\{0\}$ define $g \in L^{I} \cap C_{0}(R)$ by

$$
\hat{g}(x)= \begin{cases}\hat{f}(\exp (i x)) & (|x| \leq \pi) \\ 0 & (|x|>\pi) .\end{cases}
$$

(see Theorem C.3.5). Clearly $\hat{g}$ vanishes on $T$ and hence, since $T$ is an 
$S_{p}$-set, there exists a sequence $\left(g_{n}\right) \subset L^{I} \cap C_{0}(R)$ such that each $\hat{g}_{n}$ vanishes on a neighbourhood of $T$ and

$$
\left\|g-g_{n}\right\|_{p} \rightarrow 0
$$

If, for each $x \in(-\pi, \pi]$, we define $f_{n} \in \ell^{I}(Z)$ by

$$
\hat{f}_{n}(\exp (i x))=\hat{g}_{n}(x)
$$

(see Rudin [1], Theorem 2.7.6) then Theorem C.3.5 applied to (C.3.4) gives

$$
\left\|f-f_{n}\right\|_{p} \rightarrow 0
$$

(note that each $\hat{f}_{n}$ vanishes on a neighbourhood of $\Xi$ ). Hence $\Xi$ and consequently (see Theorem C.1.4) $\Xi_{1}$ would be an $S_{p}$-set, contradicting our choice of $\Xi_{1}$. It follows that $T_{1}$ is not an $S_{p}$-set for any $p \in[1,2)$. //

We require two lemmas before proving the main result of this section.

C.3.7 LEMMA. Let $G, H$ be Hausdorff Zocally compact Abelian groups and suppose $k \in L^{I} \cap C_{0}(G \times H)$ is such that $T=\operatorname{supp}(\hat{k})$ is compact. Then the function $y \rightarrow k(x, y)(x \rightarrow k(x, y))$ is integrable over $H$ for every $x \in G$ (over $G$ for every $y \in H$ ). Furthermore the frmetions

$$
\begin{aligned}
& \phi_{1}: x \rightarrow \int_{H} k(x, y) d \lambda_{H}(y), \\
& \phi_{2}: y \rightarrow \int_{G} k(x, y) d \lambda_{G}(x)
\end{aligned}
$$

are continuous.

Proof. Since $k$ is continuous the function $y \rightarrow k(x, y)$ is continuous, and hence measurable, for every $x \in G$.

Choose $k_{1}\left(k_{2}\right)$ in $L^{I} \cap C_{0}(G) \quad\left(L^{l} \cap C_{0}(H)\right)$ such that $\hat{k}_{1}=1$ $\left(\hat{k}_{2}=1\right)$ on a neighbourhood $\nabla_{1}\left(\nabla_{2}\right)$ of $\Upsilon_{G}\left(\Upsilon_{H}\right)$, where $\Upsilon_{G}, \Upsilon_{H}$ are the projections of $T$ onto $G, H$ respectively. If we define $h$ on $G \times H \quad$ by 


$$
h((x, y))=k_{1}(x) k_{2}(y)
$$

then Hewitt and Ross $[1],(31.7)$ (b) shows that $\hat{h}=1$ on $\nabla_{1} \times \nabla_{2}$, a neighbourhood of $T$. Thus

$$
h * k=k \quad \text { l.a.e. }
$$

and, since $h * k$ and $k$ are continuous,

$$
h * k=k \text {. }
$$

Now the map $U$ of $H \times G \times H$ into $C$, defined by

$$
u[(y, s, t)]=h(x-s, y-t) k(s, t),
$$

is continuous for every $x \in G$. Furthermore

$$
\begin{aligned}
& \int_{G \times H}\left\{\int_{H}|h(x-s, y-t)||k(s, t)| d \lambda_{H}(y)\right\} d \lambda_{G} \times \lambda_{H}(s, t) \\
&=\int_{G \times H}\left\{\int_{H}\left|k_{I}(x-s)\right|\left|k_{2}(y-t)\right| d \lambda_{H}(y)\right\}|k(s, t)| d \lambda_{G} \times \lambda_{H}(s, t) \\
&=\left\|k_{2}\right\|_{I} \int_{G \times H}\left|k_{I}(x-s)\right||k(s, t)| d \lambda_{G} \times \lambda_{H}(s, t) \\
& \leq\left\|k_{2}\right\|_{I}\left\|k_{I}\right\|_{\infty}\|k\|_{I} .
\end{aligned}
$$

Appealing to $(C .3 .5)$ and Hewitt and Ross [1], (13.4) we see that

$$
\begin{aligned}
\int_{H}|k(x, y)| d \lambda_{H}(y) & \\
& \leq \int_{H}\left\{\int_{G \times H}|h(x-s, y-t)||k(s, t)| d \lambda_{G} \times \lambda_{H}(s, t)\right\} d \lambda_{H}(y) \\
& =\int_{G \times H}\left\{\int_{H}|h(x-s, y-t)||k(s, t)| d \lambda_{H}(y)\right\} d \lambda_{G} \times \lambda_{H}(s, t)
\end{aligned}
$$

is finite for all $x \in G$, proving the first part of the lemma. Having thus also shown that $U$ is integrable on $H \times G \times H$ we can use Hewitt and Ross $[1],(13.8)$ to deduce that

$$
\phi_{1}(x)=\int_{H} k_{2}(y) d \lambda_{H}(y) \int_{G \times H} k_{1}(x-s) k(s, t) d \lambda_{G} \times \lambda_{H}(s, t) .
$$

Since $k \in L^{l}(G \times H), k_{2} \in L^{l}(H)$ and $k_{1}$ is uniformly continuous it follows that $\phi_{1}$ is continuous. 
The continuity of $\phi_{2}$ is proved similarly. //

C.3.8 LEMMA. Suppose $G, H$ are Hausdorff Zocally compact Abelian groups, with character groups $\Gamma, \Lambda$ respectively. If $p \in[1,2)$ and the closed set $\Xi^{\prime} \subset \Gamma$ is not an $S_{p}$-set, then $\Xi=\Xi^{\prime} \times \Lambda$ is not an $S_{p}$ set in $\Gamma \times \Lambda$.

Proof. Suppose to the contrary that $\Xi$ is an $S_{p}$-set in $\Gamma \times \Lambda$. Let $f \in L^{I} \cap C_{0}(G)$ with $\operatorname{supp}(\hat{f})$ compact and $\hat{f}$ vanishing on $\Xi$, and choose $g \in L^{1} \cap C_{0}(H)$ such that $\operatorname{supp}(\hat{g})$ is compact and $|g(y)| \geq I$ for all $y$ in some neighbourhood $V$ of zero in $H$. Define $h$ on $G \times H$ by

$$
h((x, y))=f(x) g(y) \text {. }
$$

Then, by Hewitt and Ross [1], (31.7) (b), $\operatorname{supp}(\hat{h})$ is compact and

$$
\begin{aligned}
\hat{h}\left(\left[r_{1}, r_{2}\right]\right) & =\hat{f}\left(r_{1}\right) \hat{g}\left(r_{2}\right) \\
& =0
\end{aligned}
$$

for all $\left[\gamma_{1}, \gamma_{2}\right] \in \Xi$.

Let $\varepsilon>0$ be given. Since $\Xi$ is assumed to be an $S_{p}$-set we can find $k \in L^{l} \cap C_{0}(G \times H)$ such that $\operatorname{supp}(\hat{k})$ is compact and disjoint from $\Xi$, and

$$
\|h-k\|_{p}<\varepsilon \lambda_{H}(V)^{I / p}
$$

Thus, for all $\gamma_{1}$ in some neighbourhood $\nabla$ of $\Xi^{\prime}$ and for all $\gamma_{2} \in \Lambda$, we have (see Hewitt and Ross [1], (13.8))

$$
\begin{aligned}
\int_{H}\left\{\int_{G} k(x, y) \bar{\gamma}_{I}(x) d \lambda_{G}(x)\right\} \bar{\gamma}_{2}(y) d \lambda_{H}(y) & \\
& =\int_{G \times H} k(x, y)\left(\left[\gamma_{I}, \gamma_{2}\right]\right)^{-}(x, y) d \lambda_{G} \times \lambda_{H}(x, y) \\
& =0 .
\end{aligned}
$$




$$
\int_{G} k(x, y) \bar{\gamma}_{I}(x) d \lambda_{G}(x)=0 \quad \lambda_{H}-\text { a.e. }
$$

Now

$$
\psi:(x, y) \rightarrow k(x, y) \bar{\gamma}_{1}(x)
$$

is contiruous and integrable, and $\operatorname{supp}(\hat{\psi})$ is compact. Hence, by Lemma C.3.7, the function $\phi$ on $H$ defined by

$$
\phi(y)=\int_{G} \psi(x, y) d \lambda_{G}(x)
$$

is continuous and so, for all $y \in H$ and $\gamma_{1} \in \nabla$,

$$
\int_{G} k(x, y) \bar{\gamma}_{l}(x) d \lambda_{G}(x)=0
$$

Using (c.3.6), we see that

$$
W=\left\{y \in V: \int_{G}|h(x, y)-k(x, y)|^{p} d \lambda_{G}(x)<\varepsilon^{p}\right\}
$$

has the property that $\lambda_{H}(V \backslash W)<\lambda_{H}(V)$, that is, $\lambda_{H}(W)>0$. Choose any $y_{0} \in W \quad(W$ is non-empty $)$. Then

$$
\begin{aligned}
\int_{G}\left|f(x)-g\left(y_{0}\right)^{-1} k\left(x, y_{0}\right)\right|^{p} d \lambda_{G}(x) & <\varepsilon^{p}\left|g\left(y_{0}\right)\right|^{-1} \\
& \leq \varepsilon^{p}
\end{aligned}
$$

and so, defining $f_{1}$ on $G$ by

$$
f_{1}(x)=g\left(y_{0}\right)^{-1} k\left(x, y_{0}\right)
$$

(c.3.7) shows that $\hat{f}_{1}$ vanishes on $\nabla$ and, from (c.3.8),

$$
\left\|f-f_{l}\right\|_{p}<\varepsilon
$$

It is clear since $k \in L^{I} \cap C_{0}(G \times H)$ that $f_{1} \in L^{I} \cap C_{0}(G)$; thus we have a contradiction of the assumption that $\Xi^{\prime}$ is not an $S_{p}$-set.

C.3.9 THEOREM. Let $G$ be a Hausdorff non-compact locally compact Abelian group, $\Gamma$ its character group. Then $\Gamma$ contains a closed set which is not an $S_{p}$-set for any $p \in[1,2)$. 
Proof. By Hewitt and Ross [1], (24.30), $\Gamma$ is topologically isomorphic with $R^{n} \times \Gamma_{0}$, where $\Gamma_{0}$ is a Hausdorff locally compact Abelian group containing a compact open subgroup.

If $n \geq 1$ then Theorem C.3.6 and Lemma C.3.8 combine to show that $\mathrm{R}^{n} \times \Gamma_{0}$ contains a closed set which is not an $S_{p}$-set for any $p \in[1,2)$. If $n=0$ then $\Gamma$ contains a compact open subgroup (which is infinite since $\Gamma$ is non-discrete) and the result follows from Corollary C.3.4. // 


\section{REFERENCES}

Bernstein, Serge

[1] "Sur l'ordre de la meilleure approximation des fonctions continues par des polynômes de degré donné", Acad. Roy. Belg. CZ. Sci. Mém. Colz. 4 (1912), 1-103.

[2] "Sur une propriété des fonctions entières", C.R. Acad. Sci. Paris Sér. $A-B 176$ (1923), 1603-1605.

Bloom, Walter R.

[1] "Bernstein's inequality for locally compact Abelian groups", J. AustraZ. Math. Soc. 17 (1974), 88-101.

[2] "A converse of Bernstein's inequality for locally compact groups", BulZ. Austral. Math. Soc. 9 (1973), 291-298.

[3] "On certain closed subsets of a locally compact Abelian group", submitted to Israel J. Math.

Boas, R.P. Jr

[1] "Inequalities for the derivatives of polynomials", Math. Mag. 42 $(1969), 165-174$.

Bourbaki, Nicolas

[1] Elements of mathematics: General topology, Parts I, II. Hermann, Éditeurs des sciences et des arts, Paris; Addison Wesley Publishing Company; Reading, Massachussetts, 1966.

Domar, Yngve

[1] "Harmonic analysis based on certain commutative Banach algebras", Acta Math. 96 (1956), 1-66.

Edwards, D.A.

[1] "On translates of $L^{\infty}$-functions", J. London Math. Soc. 36 (1961), $431-432$.

Edwards, R.E.

[1] "Translates of $L^{\infty}$ functions and of bounded measures", J. Austral. Math. Soc. 4 (1964), 403-409. 
[2] Functional analysis: Theory and applications. Holt, Rinehart and Winston, New York, Chicago, San Francisco, Toronto, London, 1965.

[3] "Supports and singular supports of pseudomeasures", J. Austral. Math. Soc. 6 (1966), 65-75.

[4] Integration and harmonic analysis on compact groups. London Mathematical Society Lecture Note Series, 8. Cambridge at the University Press, 1972.

Gaudry, G.I.

[1] "Quasimeasures and operators commuting with convolution", Pacific J. Math. 18 (1966), 461-476.

[2] "Multipliers of type $(p, q)$ ", Pacific J. Math. 18 (1966), 477-488. Godement, Roger

[1] "Théorèmes taubériens et théorie spectrale", Ann. Sci. École Norm. Sup. 64 (1947), 119-138.

Gosselin, R.P.

[1] "On Fourier transforms with small supports", J. Math. Anal. Appl. 13 $(1966), 166-178$

Henstock, R. and Macbeath, A.M.

[1] "On the measure of sum-sets. (1) The theorems of Brunn, Minkowski, and Lusternik", Proc. London Math. Soc. 3 (1953), 182-194.

Hewitt, Edwin and Ross, Kenneth A.

[1] Abstract harmonic analysis, I, II. Die Grundlehren der mathematischen Wissenschaften, Bände 115, 152. Academic Press, New. York; SpringerVerlag, Berlin, Göttingen, Heidelberg, 1963, 1970.

Hewitt, Edwin and Stromberg, Karl

[1] Real and abstract analysis. Springer-Verlag, Berlin, Heidelberg, New York, 1969. 
Kahane, J.-P.

[1] Séries de Fourier absolument convergentes. Ergebnisse der Mathematik und ihrer Grenzgebiete, Band 50. Springer-Verlag, Berlin, Heidelberg, New York, 1970.

Katznelson, Yitzhak

[1] An introduction to harmonic analysis. John Wiley and Sons, Inc., New York, London, Sydney, Toronto, 1968.

Kneser, Martin

[1] "Summenmengen in lokalkompakten abelschen Gruppen", Math. Z. 66 (1956), 88-110.

Markoff, A.

[1] "On a certain problem of D.I. Mendeleev" (in Russian), Utcheniya Zapiski Imperatorskoi Akademii Nauk (Russia) 62 (1889), 1-24. Mendeleev, D.

[1] Investigation of aqueous solutions based on specific gravity (in Russian). St. Petersburg, 1887.

Pontrjagin, L.S.

[1] Topologische Gruppen, Teil I. B.G. Teubner Verlagsgesellschaft; Leipzig, 1957.

Reiter, Hans

[1] Classical harmonic analysis and locally compact groups. Oxford at the Clarendon Press, 1968.

Riesz, Frédéric

[1] "Sur les polynômes trigonométriques", C.R. Acad. Sci. Paris Sér. A-B 158 (1914), 1657-1661.

Riesz, Marcel

[1] "Formule d'interpolation pour la dérivée d'un polynôme trigonométrique", C.R. Acad. Sci. Paris Sér. A-B 158 (1914), 1152-1154.

Rudin, Walter

[1] Fourier analysis on groups. Interscience Publishers, John Wiley and Sons, New York, London, 1962; 2nd printing, 1967. 
Schaeffer, A.C.

[1] "Inequalities of A. Markoff and S. Bernstein for polynomials and related functions", Bulz. Amer. Math. Soc. 47 (1941), 565-579. Zygmund, A.

[1] "A remark on conjugate series", Proc. London Math. Soc. 34 (1932), $392-400$ 